\title{
1 An atlas of gene expression variation across the Caenorhabditis elegans species
}

3 Gaotian Zhang ${ }^{1}$, Nicole M. Roberto ${ }^{1}$, Daehan Lee ${ }^{1}$, Steffen R. Hahnel', and Erik C.

4 Andersen ${ }^{1 *}$

5 1. Department of Molecular Biosciences, Northwestern University, Evanston, IL 60208,

6 USA

8 ORCID IDs:

9 0000-0001-6468-1341 (G.Z.)

10 0000-0002-0546-8484 (D.L.)

11 0000-0001-8848-0691 (S.R.H.)

12 0000-0003-0229-9651 (E.C.A.)

14 "Corresponding author:

15 Erik C. Andersen

16 Department of Molecular Biosciences

17 Northwestern University

184619 Silverman Hall

192205 Tech Drive

20 Evanston, IL 60208

21 E-mail: erik.andersen@northwestern.edu 


\section{Abstract}

Phenotypic variation in diverse organism-level traits have been studied in

24 Caenorhabditis elegans wild strains, but differences in gene expression and the 25 underlying variation in regulatory mechanisms are largely unknown. Here, we use natural 26 variation in gene expression to connect genetic variants to differences in organismal27 level traits, including drug and toxicant responses. We performed transcriptomic analysis

28 on 207 genetically distinct $C$. elegans wild strains to study natural regulatory variation of 29 gene expression. Using this massive dataset, we performed genome-wide association

30 mappings to investigate the genetic basis underlying gene expression variation and 31 revealed complex genetic architectures. We found a large collection of hotspots 32 enriched for expression quantitative trait loci across the genome. We further used 33 mediation analysis to understand how gene expression variation could underlie 34 organism-level phenotypic variation for a variety of complex traits. These results reveal 35 the natural diversity in gene expression and possible regulatory mechanisms in this 36 keystone model organism, highlighting the promise of gene expression variation in 37 shaping phenotypic diversity. 


\section{Introduction}

Quantitative genetic mapping approaches, such as genome-wide association (GWA) and linkage mapping, have been used in a variety of organisms to disentangle the underlying genetic basis of gene expression variation by considering the expression level of each gene as a quantitative trait $^{1-9}$. Expression quantitative trait loci (eQTL) affecting gene expression are often classified into local eQTL (located close to the genes that they influence) and distant eQTL (located further away from the genes that they influence) ${ }^{10,11}$.

47 Local eQTL are abundant in the genome. For example, over half the genes in yeast and $94.7 \%$ of all protein-coding genes in human tissues are hypothesized to have associated local eQTL ${ }^{7,8}$. Genetic variants underlying local eQTL might influence the expression of a specific gene by affecting transcription factor binding sites, chromatin accessibility, other promoter elements, enhancers, or other factors at post-transcriptional levels ${ }^{12}$. Genes encoding diffusible factors, such as transcription factors, chromatin cofactors, and RNAs, are often considered the most likely genes to underlie distant eQTL. Distant eQTL hotspots in several species have been suggested to account for the variation in expression of many genes located throughout the genome $e^{2,3,7,9,13}$. Although a substantial amount of eQTL have been identified in different species, it is still largely unknown how gene expression variation relates to organism-level phenotypic differences.

The nematode Caenorhabditis elegans is a powerful model to study the genetic basis of natural variation in diverse quantitative traits ${ }^{14-16}$. Genome-wide gene expression variation in different developmental stages and various conditions at the whole-organism or cellular resolution have been discovered and thousands of eQTL have been identified in several studies over the past two decades ${ }^{3,9,17-23}$. However, most of these studies used

63 two-parent recombinant inbred lines derived from crosses of the laboratory-adapted reference strain, N2, and the genetically diverse Hawaiian strain, CB4856. Consequently, the observed variation in gene expression and their identified eQTL were limited to the differences among a small number of $C$. elegans strains and only revealed a tiny fraction

67 of the natural diversity of gene expression and regulatory mechanisms in this species.

68 The C. elegans Natural Diversity Resource (CeNDR) has a collection of 540 genetically distinct wild C. elegans strains ${ }^{16,24,25}$. Variation in diverse organism-level phenotypes has 
70 been observed among these wild strains, and many underlying QTL, quantitative trait

71 genes (QTGs), and quantitative trait variants (QTVs) have been identified using GWA

72 mappings ${ }^{15,16}$. Therefore, a genome-wide analysis could improve our understanding of

73 the role of gene regulation in shaping organism-level phenotypic diversity, adaptation,

74 and evolution of C. elegans.

$75 \quad$ Here, we investigated the natural variation in gene expression of 207 genetically

76 distinct $C$. elegans wild strains by performing bulk mRNA sequencing on synchronized

77 young adult hermaphrodites. We used GWA mappings to identify 6,545 eQTL associated

78 with variation in expression of 5,291 transcripts of 4,520 genes. We found that local eQTL

79 explained most of the narrow-sense heritability and showed larger effects on expression

80 variation than distant eQTL. We identified 67 hotspots that comprise 1,828 distant eQTL

81 across the $C$. elegans genome. We further found a diverse collection of potential 82 regulatory mechanisms that underlie these distant eQTL hotspots. Additionally, we

83 applied mediation analysis to gene expression and other quantitative trait variation data

84 to elucidate putative mechanisms that can play a role in organism-level trait variation.

85 Our results provide an unprecedented resource of transcriptome profiles and genome86 wide regulatory regions that facilitate future studies. Furthermore, we demonstrate 87 efficient methods to locate causal genes that underlie mechanisms of organism-level 88 trait differences across the $C$. elegans species.

\section{Results}

\section{Transcriptome profiles of 207 wild C. elegans strains}

We obtained 207 wild C. elegans strains from CeNDR $^{25}$ (Fig. 1a). We grew and

92 harvested synchronized populations of each strain at the young adult stage in 93 independently grown and prepared biological replicates (Fig. 1b). We performed bulk 94 RNA sequencing to measure expression levels and aligned reads to strain-specific 95 transcriptomes (Fig. 1b, Supplementary Fig. 1, Supplementary Data 1). We focused on 96 protein-coding genes and pseudogenes and filtered out those genes with low and/or 97 rarely detected expression (See Methods). Because various hyper-divergent regions with 
extremely high nucleotide diversity were identified in the genomes of wild $C$. elegans strains $^{26,27}$, RNA sequencing reads might be poorly aligned and expression abundances might be underestimated for genes in these regions. For each strain, we filtered out transcripts that fell into the known hyper-divergent regions. We also dropped outlier samples by comparing sample-to-sample expression distances (Supplementary Fig. 1). To further verify the homogeneity of developmental stages of our samples, we evaluated the age of each sample when they were harvested using our expression data and published time-series expression data ${ }^{28}$. We inferred that our animals fit an expected developmental age of 60 to 72 hours post hatching (Fig. 1c), during which time the animal is in the young adult stage. Because we harvested the animals at the first embryo-laying event, the age estimation also reflects natural variation in the duration from hatching to the beginning of embryo-laying of wild $C$. elegans. In summary, we obtained reliable expression abundance measurements for 25,849 transcripts from 16,094 genes (15,364 protein-coding genes and 730 pseudogenes) in 561 samples of 207 C. elegans strains

112 (Fig. 1b, Supplementary Fig. 1, Supplementary Data 1), which we used for downstream 113 analyses.

C. elegans geographic population structure has been observed previously ${ }^{24,27,29}$. Wild strains from Hawaii and other regions in the Pacific Rim harbor high genetic diversity and group into distinct clusters using genetic relatedness and principal component analysis $^{24,27,29}$. Other strains that were isolated largely from Europe have relatively low genetic diversity because of the recent selective sweeps ${ }^{24,27,29}$. Similar to the previous results, the 207 strains were classified into three major groups consisting of strains from Hawaii, the Pacific coast of the United States, and Europe, respectively, in the genetic relatedness tree (Fig. 1d). Three Hawaiian strains are extremely divergent from all other

122 strains. However, a tree constructed using transcriptome data only exhibited weak 123 geographic relationships and no highly divergent strains (Fig. 1e), suggesting stabilizing selection has constrained variation in gene expression. 
a

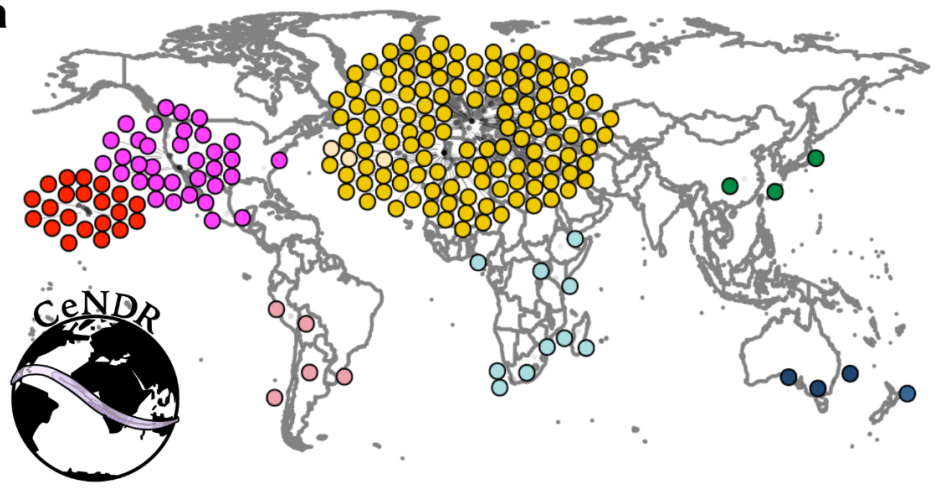

c.

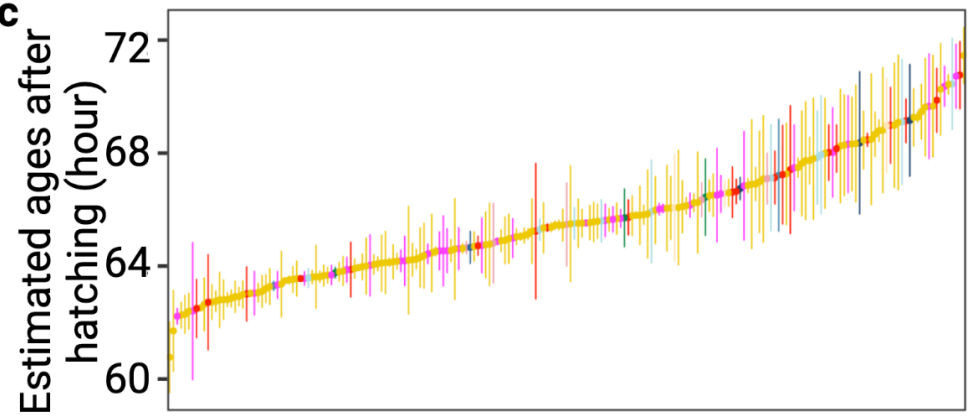

$$
\text { 山 }
$$$$
\text { d }
$$

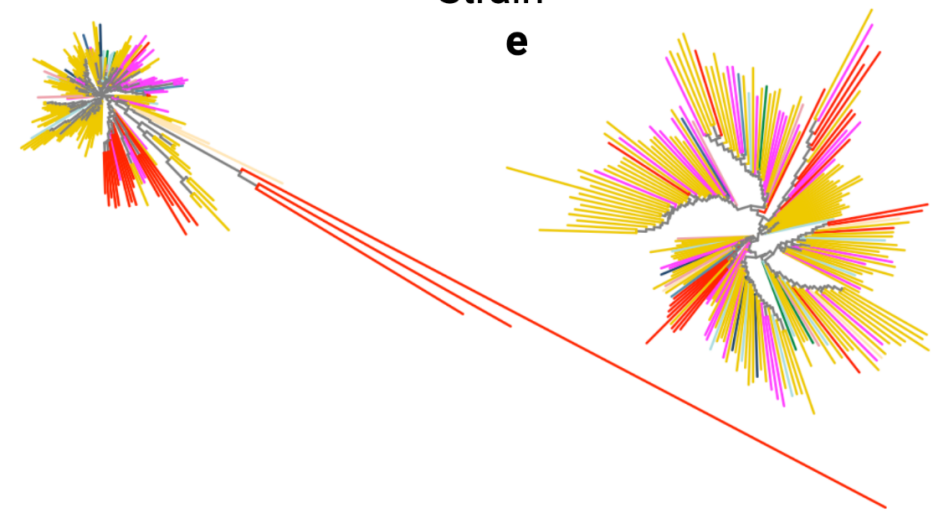

b

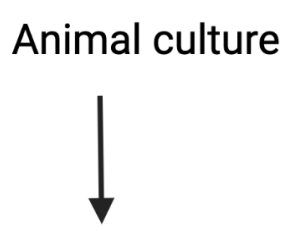

RNA-seq
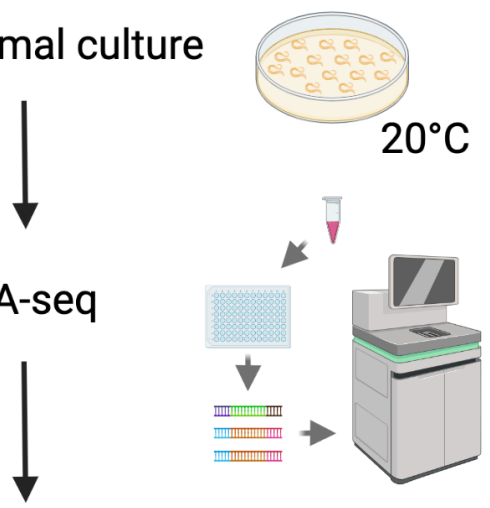

Alignment
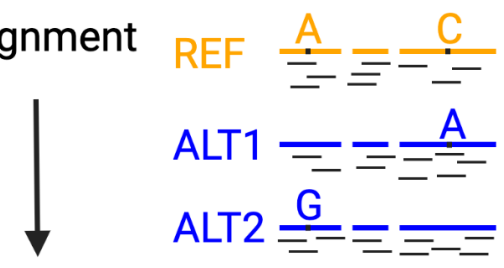

ALT1 $=\equiv \underline{\underline{A}}$

ALT2 $\stackrel{\mathrm{G}}{=}=\overline{=-}$

Filtering Reliable expression

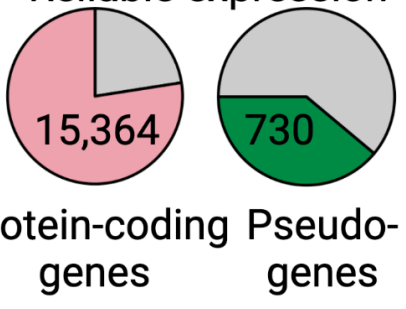

GWA mapping

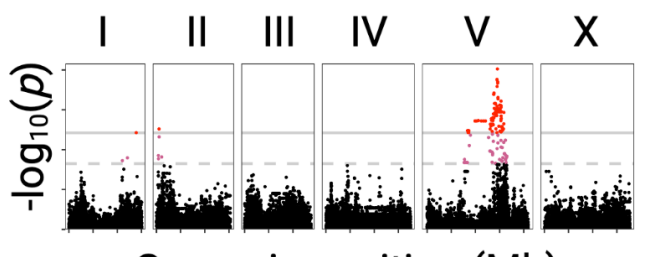

Genomic position $(\mathrm{Mb})$

Fig. 1: Overview of species-wide expression analysis in wild C. elegans.

a Global distribution of 205 of the 207 wild C. elegans strains that were obtained from CeNDR and used in this study. Strains are colored by their sampling location continent, except for Hawaiian strains (in red). The two strains missing on the map are lacking sampling locations. b Graphic illustration of the workflow to acquire C. elegans transcriptome data. Created using BioRender.com. c Estimated developmental age ( $y-$ axis) of 561 well clustered samples of the 207 wild C. elegans strains (x-axis). Strains on the $x$-axis are sorted by their mean estimated age from two to three biological replicates. Error bars show standard deviation of estimated age among replicates of each strain. $\mathbf{d}$, e Two Neighbor-joining trees of the 207 C. elegans strains using 851,105 biallelic segregating sites (d) and expression of 22,268 transcripts (e) are shown. Strains in c, d, e are colored as in a. 


\section{Complex regulatory genetic architectures in wild C. elegans strains}

143

144

To estimate the association between gene expression differences and genetic variation, we calculated the broad-sense heritability $\left(H^{2}\right)$ and the narrow-sense heritability $\left(h^{2}\right)$ for each of the 25,849 transcript expression traits. We observed a median $H^{2}$ of 0.31 and a median $h^{2}$ of 0.06 (Fig. 2a, Supplementary Data 1), indicating strong influences from environmental factors, epistasis, or other stochastic factors on transcript expression variation ${ }^{7,30,31}$. Nearly 4,000 traits have a $h^{2}$ higher than 0.18 , indicating a substantial heritable genetic component of the population-wide expression differences.

We performed marker-based GWA mappings to investigate the genetic basis of expression variation in the 25,849 transcripts (Supplementary Data 1). We determined the $5 \%$ false discovery rate (FDR) significance threshold for eQTL detection by mapping 40,000 permuted transcript expression traits using the EMMA algorithm ${ }^{32}$ and the eigendecomposition significance (EIGEN) threshold ${ }^{33}$ (See Methods). In total, we detected 6,545 significant eQTL associated with variation in expression of 5,291 transcripts from 4,520 genes (Fig. 2b, Supplementary Data 2). The correlation of $h^{2}$ and $H^{2}$ among traits with $\mathrm{eQTL}$ is much higher than among traits without eQTL (Kendall's t coefficient, 0.45 and 0.27 , respectively) (Fig. 2a), indicating major roles of additive genetic variation on expression variation than other genetic factors. Likely because GWA mappings mainly detect QTL that contribute additively to trait variance, eQTL were detected for $71 \%$ of the traits with $h^{2}>0.18$, but only $11 \%$ of the remaining traits (Fig. 2a).

In close agreement to previous $C$. elegans $\mathrm{eQTL}$ studies using recombinant inbred advanced intercross lines (RIAILs) derived from a cross of the N2 and CB4856 strains ${ }^{3,9}$, eQTL in this study were mostly found on chromosome arms $(61 \%)$ relative to centers (33\%), which is likely related to the genomic distribution of genomic variation (Table 1). Of the 4,520 genes with transcript-level eQTL, we found overrepresentation of nonessential genes (Fisher exact test, odds ratio: 1.18, $p$-value: 0.001 ) and underrepresentation of essential genes (Fisher exact test, odds ratio: $0.75, p$-value: 0.001 ), suggesting stronger selection against expression variation in essential genes than nonessential genes ${ }^{34}$. Gene set enrichment analysis (GSEA) on these 4,520 genes showed that proteolysis proteasome-related genes (Fisher Exact Test, Bonferroni FDR 
172 corrected $p=3.76 \mathrm{E}-20)$, especially genes encoding E3 ligases containing an F-box

173 domain (Fisher Exact Test, Bonferroni FDR corrected $p=3.73 \mathrm{E}-15$ ), are the most

174 significantly enriched class (Supplementary Fig. 2, Supplementary Data 3). Other

175 significantly enriched gene classes include metabolism (Fisher Exact Test, Bonferroni

176 FDR corrected $p=2.92 \mathrm{E}-12$ ), stress response (Fisher Exact Test, Bonferroni FDR

177 corrected $p=7.24 \mathrm{E}-12$ ), and histones (Fisher Exact Test, Bonferroni FDR corrected $p=$

178 3.23E-8). (Supplementary Fig. 2).

179 We classified eQTL located within a two megabase region surrounding each 180 transcript as local eQTL and all other eQTL as distant ${ }^{3,9}$ (Fig. 2b, Table 1, Supplementary 181 Data 2). We identified local eQTL for 3,185 transcripts from 2,655 genes (Fig. 2b, Table 182 1, Supplementary Data 2). The 2,551 local eQTL that passed the Bonferroni 5\% FDR 183 threshold explained most of the estimated narrow-sense heritability (Fig. 2c). 184 Additionally, we found 3,360 distant eQTL for 2,553 transcripts from 2,382 genes (Fig. 185 2b, Table 1, Supplementary Data 2). Compared to local eQTL, distant eQTL generally 186 explained significantly lower variance (Fig. 2c, d). We found that local eQTL and up to 187 six distant eQTL could jointly regulate the expression of transcripts (Fig. 2e). Because 188 substantial linkage disequilibrium (LD) is observed within $\left(r^{2}>0.6\right)$ and between $\left(r^{2}>\right.$ 189 0.2) chromosomes in wild C. elegans strains ${ }^{24,27,35}$, we calculated LD among eQTL of 190 each of the 861 transcripts with multiple eQTL. We found low LD among most eQTL, 191 with a median LD of $r^{2}=0.19$ (Supplementary Fig. 3), suggesting complex genetic 192 architectures underlying variation in expression of these transcripts are driven by 193 independent loci. 

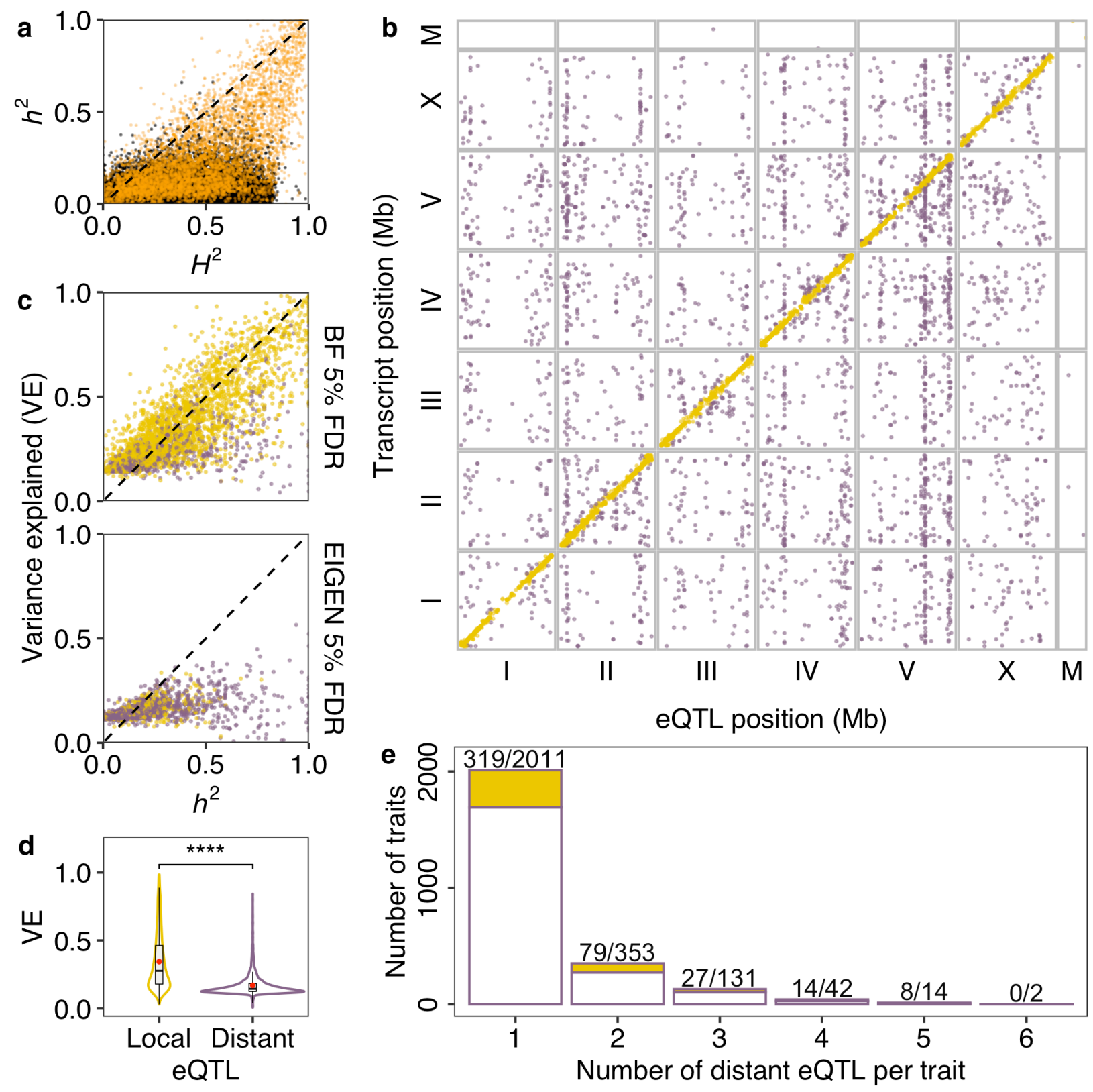

Fig. 2: Expression QTL map of 207 wild C. elegans strains.

a Heritability for 25,849 transcript expression traits with (orange) or without (black) detected eQTL. The narrow-sense heritability $\left(h^{2}, y\right.$-axis) for each trait is plotted against the broad-sense heritability $\left(H^{2}, x\right.$-axis). b The genomic locations of 6,545 eQTL peaks (x-axis) that pass the genome-wide EIGEN 5\% FDR threshold are plotted against the genomic locations of the 5,291 transcripts with expression differences (y-axis). Golden points on the diagonal of the map represent local eQTL that colocalize with the transcripts that they influence. Purple points correspond to distant eQTL that are located further away from the transcripts that they influence. $c$ The variance explained (VE) by each detected eQTL (y-axis) that passed Bonferroni (BF) 5\% FDR or EIGEN 5\% FDR threshold for each trait is plotted against the narrow-sense heritability $h^{2}$ (x-axis). The 
dashed lines on the diagonal are shown as visual guides to represent $h^{2}=H^{2}$ (a) and VE $=h^{2}$ (c). $\mathbf{d}$ Comparison of VE between detected local and distant eQTL shown as Violin plots. The mean VE by local or distant eQTL is indicated as red points. Statistical significance was calculated using the Wilcoxon test with $p$-value $<2 e-16$ indicated as ${ }^{* \star * *}$. e A histogram showing the number of distant eQTL detected per transcript expression trait. One to six distant eQTL were detected for 2,553 transcript expression traits, of which 447 traits also have one local eQTL. Numbers before slashes (indicated as the golden proportion of each bar) represent the number of traits with a local eQTL in addition to their distant eQTL. Numbers after each slash on top of each bar represent the total number of traits in each category.

Table 1: The distribution of eQTL and SNVs.

220 Genomic domain coordinates were defined previously ${ }^{36}$. Transcript expression traits 221 and SNVs used for eQTL mappings were listed.

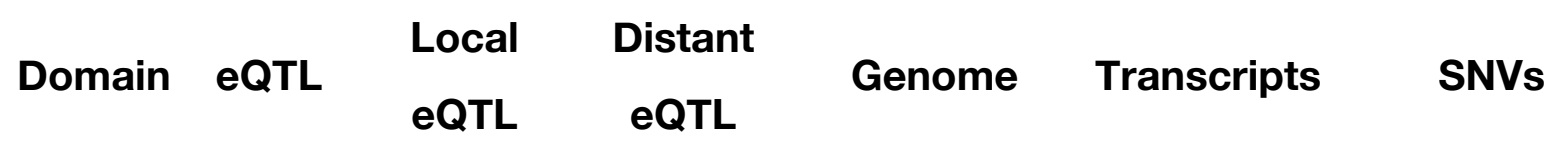

\begin{tabular}{lcccccc}
\hline Tip & 388 & 224 & 164 & $7.37 \mathrm{Mb}$ & 1,712 & 1,628 \\
& $(5.93 \%)$ & $(7.03 \%)$ & $(4.88 \%)$ & $(7.35 \%)$ & $(6.62 \%)$ & $(7.76 \%)$ \\
& 3,966 & 2,027 & 1,939 & $45.89 \mathrm{Mb}$ & 9,503 & 12,883 \\
Arm & $(60.60 \%)$ & $(63.64 \%)$ & $(57.71 \%)$ & $(45.76 \%)$ & $(36.76 \%)$ & $(61.37 \%)$ \\
& 2,183 & 932 & 1,251 & $47.01 \mathrm{Mb}$ & 14,622 & 6,429 \\
Center & $(33.35 \%)$ & $(29.26 \%)$ & $(37.23 \%)$ & $(46.88 \%)$ & $(56.57 \%)$ & $(30.63 \%)$ \\
& 8 & 2 & 6 & $0.01 \mathrm{Mb}$ & 12 & 51 \\
MtDNA & $(0.12 \%)$ & $(0.06 \%)$ & $(0.18 \%)$ & $(0.01 \%)$ & $(0.05 \%)$ & $(0.24 \%)$ \\
& 6,545 & 3,185 & 3,360 & $100.29 \mathrm{Mb}$ & 25,849 & 20,991 \\
\hline
\end{tabular}
distant eQTL, 1,828 were clustered into 67 hotspots, each of which affected the 227 expression of 12 to 184 transcripts (Fig. 3). Signatures of selection (Tajima's $D$ values) in 
228 hotspots are mostly negative, likely because of the recent selective sweeps

229 (Supplementary Fig. 4) $)^{24}$.

230 GSEA on genes with transcript-level distant eQTL in each hotspot revealed

231 potential shared transcriptional regulatory mechanisms across different genes of the

232 same class (Supplementary Fig. 5, Supplementary Data 3). For example, the hotspot at

$23321.5 \mathrm{cM}$ on chromosome II significantly affected the expression of heat stress related

234 genes (Fisher Exact Test, Bonferroni FDR corrected $p=7.03 \mathrm{E}-7$ ). Our results also

235 showed that a single hotspot could regulate expression of genes in different classes.

236 The hotspot at $2.5 \mathrm{cM}$ on chromosome II significantly affected the expression of genes

237 in three classes, including metallopeptidases (Fisher Exact Test, Bonferroni FDR

238 corrected $p=1.31 \mathrm{E}-5$ ), collagen proteins (Fisher Exact Test, Bonferroni FDR corrected

$239 p=3.11 \mathrm{E}-9$ ), and histones (Fisher Exact Test, Bonferroni FDR corrected $p=1.26 \mathrm{E}-6$ )

240 (Supplementary Fig. 5, Supplementary Data 3). Furthermore, different hotspots could

241 affect the expression of the same gene class. For example, the hotspot at $45.5 \mathrm{cM}$ on

242 chromosome III was also enriched with distant eQTL of histones (Fisher Exact Test,

243 Bonferroni FDR corrected $p=8.2 \mathrm{E}-7)$ like the hotspot at 2.5 on chromosome II

244 (Supplementary Fig. 5, Supplementary Data 3). Regulatory genes, such as transcription

245 factors and chromatin cofactors, that are located in each hotspot could underlie the

246 regulation of multiple genes. We found previously known or predicted genes encoding

247 chromatin cofactors and transcription factors ${ }^{37-39}$ in 24 and 59 of the 67 hotspots,

248 respectively (Supplementary Fig. 6).

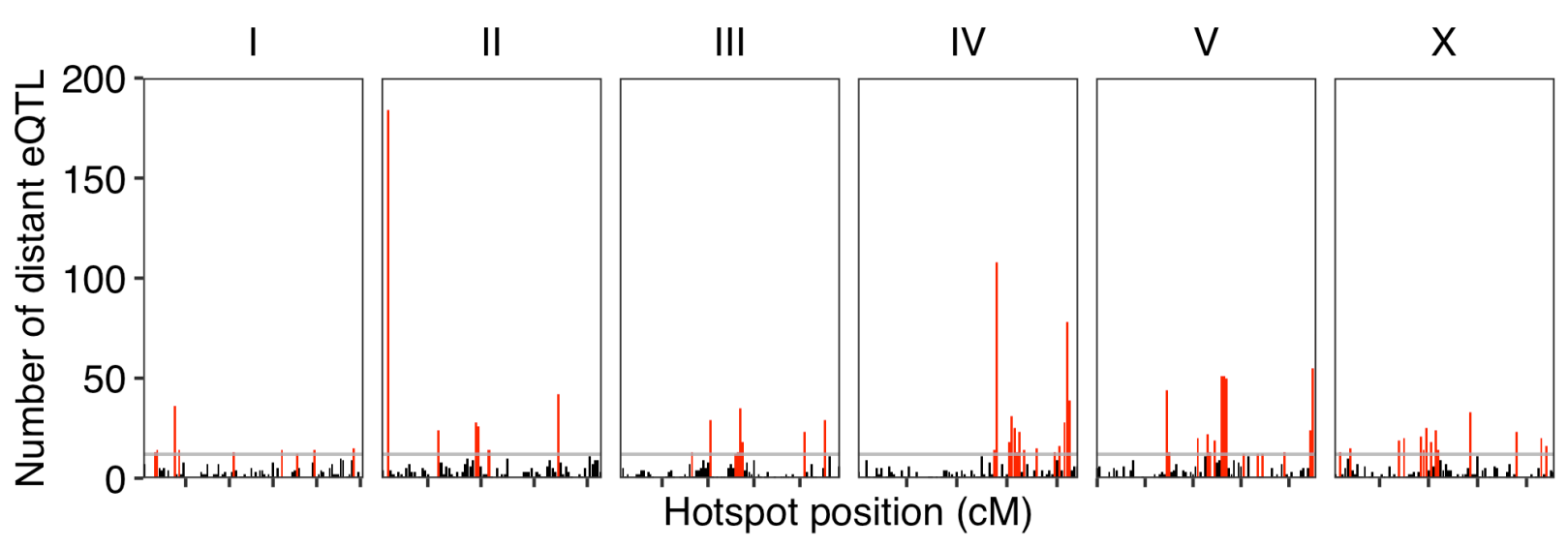




\section{Fig. 3: Distant eQTL hotspots.}

The number of distant eQTL ( $y$-axis) in each $0.5 \mathrm{cM}$ bin across the genome ( $\mathrm{x}$-axis) is shown. Tick marks on the $x$-axis denote every $10 \mathrm{cM}$. The horizontal gray line indicates the threshold of $12 \mathrm{eQTL}$. Bins with 12 or more eQTL were identified as hotspots and are colored red. Bins with fewer than $12 \mathrm{eQTL}$ are colored black.

To identify causal genes and variants underlying hotspots, we performed fine mapping on distant eQTL in each hotspot and filtered for the most likely candidate variants (see Methods for details) (Supplementary Data 4). Then, we focused on the filtered candidate variants that were mapped for at least four traits in each hotspot and are in genes encoding transcription factors or chromatin cofactors. In total, we identified 36 candidate genes encoding transcription factors or chromatin cofactors for 34 hotspots. For example, the gene $t t x-1$, which encodes a transcription factor necessary for thermosensation in the AFD neurons ${ }^{40,41}$, might underlie the expression variation of 97 transcripts with distant eQTL in three hotspots between $44.5 \mathrm{cM}$ and $45.5 \mathrm{cM}$ on chromosome V. TTX-1 regulates expression of gcy-8 and gcy-18 in AFD neurons ${ }^{40,41}$, but no eQTL were detected for the two genes likely because we measured the expression

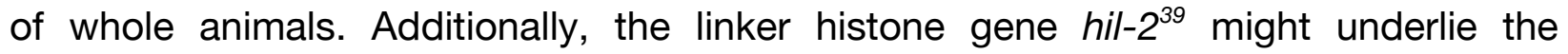
expression variation of $46,10,17$, and four transcripts with distant eQTL in the hotspots at $28 \mathrm{cM}, 30.5 \mathrm{cM}, 31 \mathrm{cM}$ and $31.5 \mathrm{cM}$, respectively, on chromosome IV. We also performed GSEA for groups of transcripts whose expression traits were fine mapped to

272 the 36 candidate genes encoding transcription factors or chromatin cofactors. For 273 instance, the 17 traits that fine mapped to hil-2 in the hotspot at $31 \mathrm{cM}$ on chromosome 274 IV (Supplementary Fig. 7) were enriched in E3 ligases containing an F-box domain (Fisher

275 Exact Test, Bonferroni FDR corrected $p=0.0003$ ) and transcription factors of the 276 homeodomain class (Fisher Exact Test, Bonferroni FDR corrected $p=0.002$ ). Besides 277 the 36 candidate genes, the hundreds of other fine mapping candidates are not as 278 transcription factors or chromatin cofactors, suggesting other mechanisms underlying 279 distant eQTL. Altogether, as previously implicated in other species ${ }^{7,11,42}$, our results 280 indicate that a diverse collection of molecular mechanisms likely cause gene expression 281 variation in C. elegans. 


\section{Mediation analysis facilitates candidate gene prioritization}

Mediation analysis seeks to identify the mechanism that underlies the relationship between an independent variable and a dependent variable via the inclusion of an intermediary mediating variable. Because gene expression has been found to play an intermediate role between genotypes and phenotypes, it could help to identify the causal mediating genes between genotypes and phenotypes in quantitative genetics mapping studies. We have previously identified mediation effects of $s c b-1$ expression on responses to several chemotherapeutics and sqst-5 expression on differential responses to exogenous zinc using linkage mapping experiments ${ }^{9,43}$. To validate if our expression and EQTL data can be used to identify candidate genes, we first performed mediation analysis on one published GWA study of variation in responses to the commonly used anthelmintic albendazole $(A B Z)^{44}$.

Previously, wild C. elegans strains were exposed to $A B Z$ and measured for effects on development to identify genomic regions that contribute to variation in $A B Z$ resistance. A single-marker GWA mapping was performed first to detect two QTL on chromosomes II and V, but no putative candidate gene was identified. Using a burden mapping approach, prior knowledge of $A B Z$ resistance in parasitic nematodes, and manually curation of raw sequence read alignment files, the gene ben-1 was found to underlie natural variation in ABZ resistance variation ${ }^{44}$. The single-marker GWA mapping was not able to detect an association between $A B Z$ resistance and ben-1 variation because of high allelic heterogeneity caused by rare SNVs and structural variants (Supplementary Fig. 8). However, rare SNVs or structural variants might lead to changes in ben-1 expression and $A B Z$ resistance. We found two distant $\mathrm{eQTL}$, in regions overlapping with the two organism-level ABZ QTL, for ben-1 expression variation. Therefore, these results provided an excellent opportunity to test the effectiveness of mediation analysis among organism-level phenotypes, genotype, and gene expression.

308 The mediation estimate for ben-1 expression was the second strongest hit in the analysis 309 on the phenotype (animal length in response to $A B Z$ ), the genotype (GWA QTL of the 310 phenotype), and the expression of 1,157 transcripts (Fig. 4a). We found a moderate 311 negative correlation between the expression of ben-1 and animal length and almost no 
312 correlation after we regressed animal length by the expression of ben-1 (Fig. 4b), 313 suggesting that expression variation impacts differences in $A B Z$ responses. We further

314 examined genetic variants across strains and found that those strains with relatively low

315 ben-1 expression and high ABZ resistance all harbor SNVs or structural variants with

316 different predicted effects (Fig. 4b), suggesting that the extreme allelic heterogeneity at

317 the ben-1 locus might affect ABZ response variation by reducing the abundance of this

318 beta-tubulin. To test the impact of expression variation on phenotypic variation, we

319 regressed animal length by expression of every transcript in our data and performed

320 GWA mappings. Then, we compared the GWA mapping significance value after

321 regression to the original GWA mapping significance value at a pseudo variant marker

322 that represents all the variants in ben-1 (Fig. 4c, Supplementary Fig. 8) ${ }^{45}$. We found

323 animal length regressed by the expression of ben-1 showed one of the largest drops in

324 significance, and significance in most of the other mappings was approximately equal to

325 the original significance value (Fig. 4c, Supplementary Fig. 8). These results indicated

326 that increasing ben-1 expression decreases resistance to ABZ and suggested the

327 applicability of mediation analysis using the expression and eQTL data for other $C$.

328 elegans quantitative traits. 


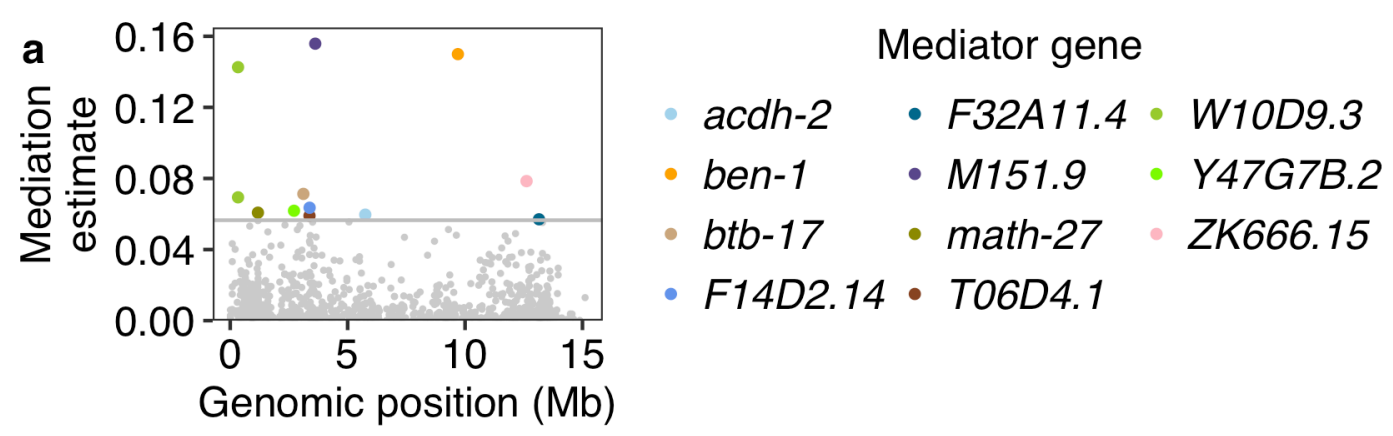

b

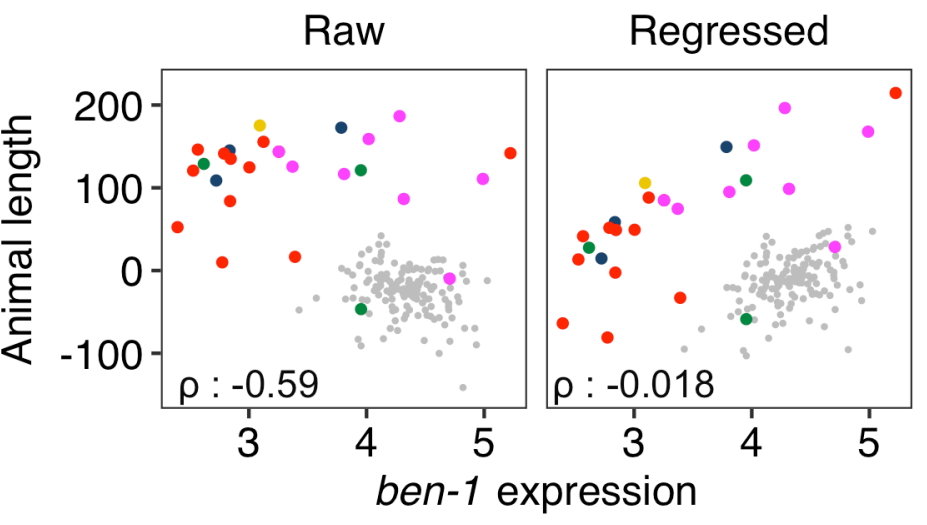

Variation at ben-1

- Deletion

- Frameshift

- Missense

- Stop gained

- Inversion

C

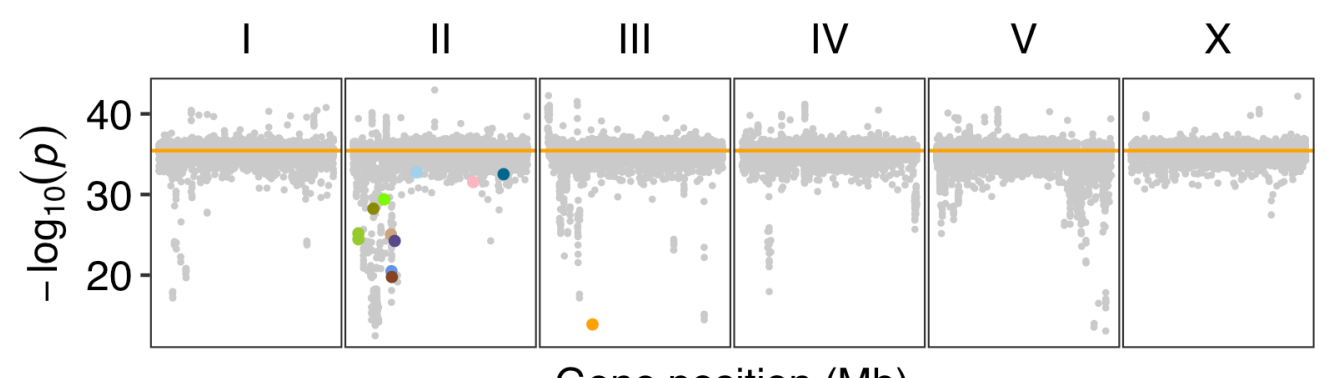

Gene position (Mb)

Fig. 4: Mediation effects of ben-1 expression on C. elegans resistance to ABZ.

a Mediation estimates ( $y$-axis) calculated as the indirect effect that differences in expression of each gene play in the overall phenotype are plotted against the genomic position of the eQTL (x-axis) on chromosome II. The horizontal gray line represents the $99^{\text {th }}$ percentile of the distribution of mediation estimates. Significant mediators are colored other than gray by their genes as shown in the legend. $\mathbf{b}$ The correlation of ben1 expression (x-axis) to raw animal length and to animal length regressed by ben-1 expression on $y$-axis. The Pearson's coefficient $\rho$ for each correlation was indicated at bottom left. Strains are colored by the type of their genetic variants in ben-1. Strains without identified variants are colored gray. c Significance at the pseudo variant marker of 25,837 GWA mappings. Each point represents a GWA mapping that is plotted with its $-\log _{10}(p)$ value (y-axis) at the pseudo variant marker (III: 3,539,640) against the genomic locations (x-axis) of the transcript of which the expression was used in regression for animal length. Points for traits regressed by expression of transcripts identified as significant mediators are colored as in (a). The orange horizontal line represents the significance at the pseudo variant marker using the raw animal length of 167 strains 
347 (Supplementary Fig. 8). GWA mapping results of 12 traits regressed by expression of 348 mitochondrial genes were excluded but all with significance close to the horizontal line.

We further applied mediation analysis to another eight previously published studies of $C$. elegans natural variation and GWA mappings in diverse traits, including telomere length $^{46}$ (Fig. 5a), responses to $\operatorname{arsenic}^{47}$ (Fig. 5b), zinc ${ }^{43}$ (Fig. 5c), etoposide ${ }^{48}$

353 (Fig. 5d), propionate ${ }^{49}$ (Fig. 5e), abamectin ${ }^{50}$ (Fig. 5f), dauer formation in response to pheromone $^{51}$, and lifetime fecundity ${ }^{52}$ (Fig. $5 \mathrm{~g}$ ). Causal variants and genes that partially explained the phenotypic variation in all the eight traits, except for lifetime fecundity, have been identified using fine mappings and genome-editing experiments ${ }^{43,46-52}$. Only one causal gene, $d b t-1$ (for arsenic response variation ${ }^{47}$ ), has eQTL detected and its expression was tested in mediation analysis for arsenic response variation ${ }^{47}$ (Fig. 5b). No significant mediation effects were found on arsenic response variation by the expression of $d b t-1$. We also did not observe significant differential expression between strains with different alleles at the previously validated causal $d b t-1$ QTV (II:7944817) ${ }^{47}$. Therefore, this causal variant possibly causes arsenic response variation only by affecting enzymatic activity ${ }^{47}$ and not the abundance of the $d b t-1$ transcript. Instead, we identified bath-15 as a significant mediator gene for arsenic response variation (Fig. 5b). For the other seven organism-level traits, putative genes whose expression likely mediated the phenotypic variation were detected for six of the traits (Fig. 5). For example, the top mediator gene for the variation in responses to abamectin was cyn-7, which is predicted to have peptidyl-prolyl cis-trans isomerase acitivity (Fig. $5 f)^{53}$. For the variation in lifetime fecundity (Fig. 5g), one of the putative mediator genes was ets-4, which is known to affect larval developmental rate, egg-laying rate, and lifespan ${ }^{54}$. Mediator genes suggest

372 mappings. Taken together, we concluded that mediation analysis using the newly 373 generated expression and eQTL data facilitates candidate gene prioritization in GWA 374 studies. 
a

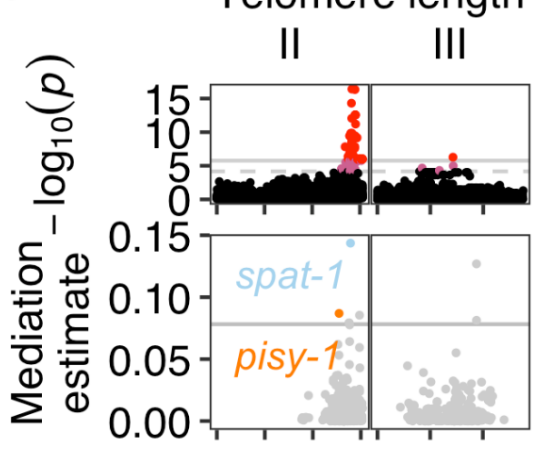

b
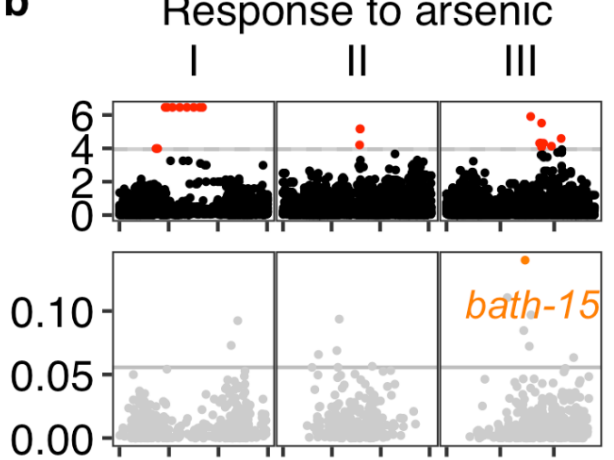

c Response to zinc III

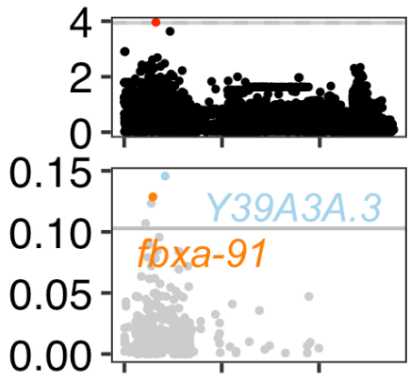

d

Response to etoposide

e

Response to propionate
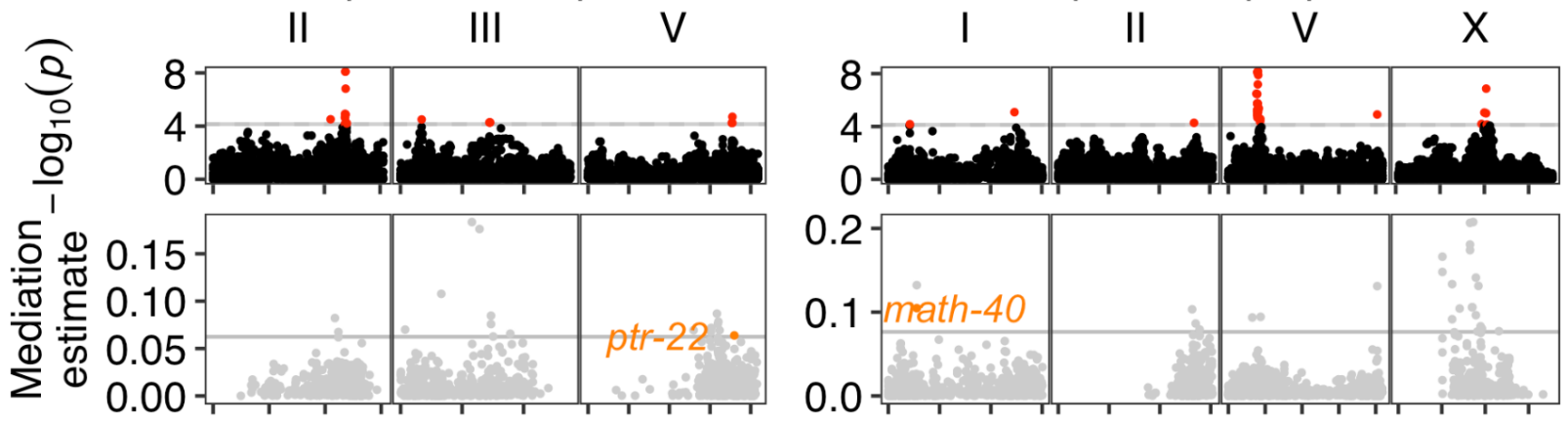

f

Response to abamectin

g

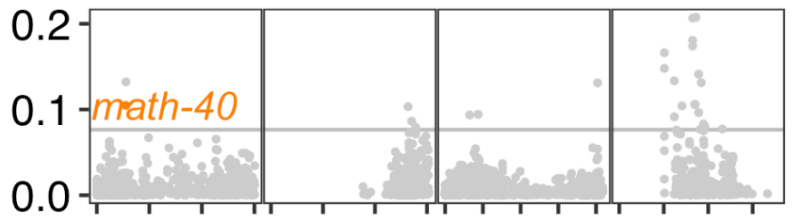

II
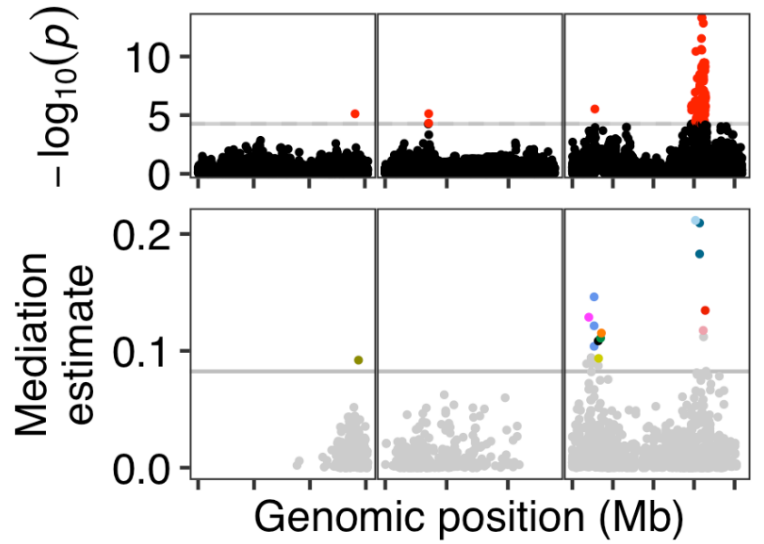

- C36C5.4 cyn-7 • F40C5.2

- F44G3.10 - F57A10.4 - folt-2

- hmg-1.1 •K08D9.9 • skr-11

-W02G9.3 - Y57G7A.8
Lifetime fecundity

II $\mathrm{V}$
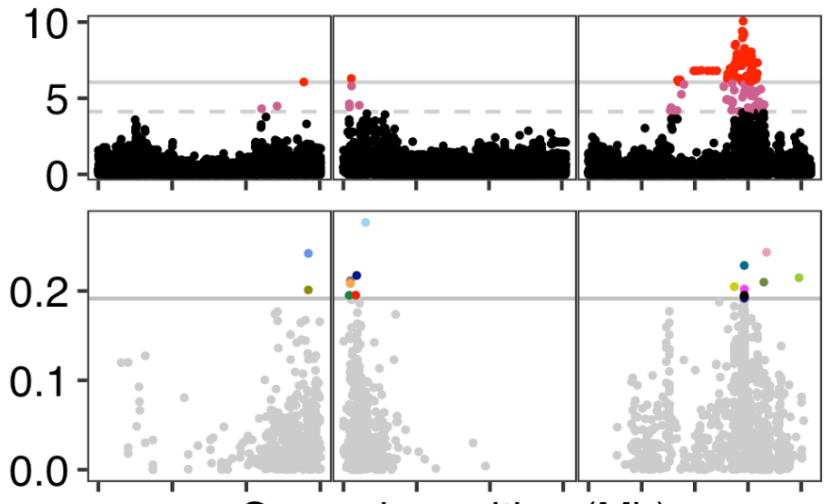

Genomic position (Mb)

Fig. 5: Mediation effects of gene expression on variation in seven organism-level phenotypes of C. elegans. 
and lifetime fecundity (g). Top panel: A Manhattan plot indicating the GWA mapping result for each phenotype is shown. Each point represents an SNV that is plotted with its genomic position (x-axis) against its $-\log _{10}(p)$ value (y-axis) in mapping. SNVs that pass the genome-wide EIGEN threshold (the dotted gray horizontal line) and the genome-wide Bonferroni threshold (the solid gray horizontal line) are colored pink and red, respectively. QTL were identified by the EIGEN (c,d,e,f) or Bonferroni $(\mathbf{a}, \mathbf{b}, \mathbf{g})$ threshold. Only chromosomes with identified QTL were shown. Bottom panel: Mediation estimates (y-axis) calculated as the indirect effect that differences in expression of each gene plays in the overall phenotype are plotted against the genomic position (x-axis) of the eQTL. The horizontal gray line represents the $99^{\text {th }}$ percentile of the distribution of mediation estimates. The mediator genes with adjusted $p<0.05$ and interpretable mediation estimate $>$ the $99^{\text {th }}$ percentile estimates threshold are colored other than gray and labeled in the panel (a-e) or below the panel $(\mathbf{f}, \mathbf{g})$. Tick marks on $\mathbf{x}$-axes denote every $5 \mathrm{Mb}$.

\section{Discussion}

C. elegans was the first metazoan to have its genome sequenced and has been subjected to numerous genetic screens to identify the genes that underlie diverse traits, including programmed cell death, drug responses, development, and behaviors. Despite huge efforts by a large research community, over $60 \%$ of its genes have not been curated with functional annotations or associated with defined mutant phenotypes ${ }^{55}$. A

402 likely reason is that most $C$. elegans research uses the reference strain N2 under 403 laboratory conditions, and the functions of many genes might only be revealed in natural 404 environments or in different genetic backgrounds ${ }^{56}$. In the last decade, wild C. elegans 405 strains have exhibited diverse phenotypic variation in natural ecology studies ${ }^{16,25,29,57-59}$. 406 Here, we provide an unprecedentedly large resource of transcriptome profiles from wild 407 C. elegans strains. We used these data and GWA mappings to study gene regulation 408 variation and detected 6,545 eQTL associated with variation in expression of 5,291 409 transcripts of 4,520 genes. These genes are enriched in processes, including the 410 proteasome, metabolism, stress response, etc., suggesting gene expression regulation 411 plays an important role in adaptation of natural $C$. elegans strains to various 412 environments ${ }^{60,61}$. We identified local eQTL that explained most of the narrow-sense 413 heritability $\left(h^{2}\right)$ and significantly larger variance than distant eQTL, likely because of 
414 higher possibilities of pleiotropy and thus stronger selection pressures. We also 415 observed lower variation in gene expression than in genome sequence and 416 underrepresentation of essential genes among all of the genes identified with eQTL, 417 suggesting stabilizing selection against gene expression as previously observed in $C$. 418 elegans and other species ${ }^{5,12,62,63}$.

$419 \quad$ Although previous C. elegans eQTL studies using recombinant inbred lines have 420 revealed rich information on the genetic basis of gene expression variation, mapping 421 using 207 genetically distinct wild strains has the advantage of much greater genetic 422 diversity. We reanalyzed results of one previous study that used linkage mapping to 423 identify eQTL from the young adult stage of N2xCB4856 recombinant inbred lines ${ }^{3,9}$. We 424 reclassified 1,208 local eQTL and 1,179 distant eQTL for 2,054 microarray probes of 4252,003 genes (Supplementary Fig. 9a). Both the eQTL GWA and linkage mappings detected overlapping local eQTL for 454 genes and distant eQTL for 19 genes, indicating 427 that the CB4856 strain carries the common alternative alleles among wild C. elegans 428 strains for these 473 loci. However, among the 6,545 eQTL that we detected, the strains 429 N2 and CB4856 shared the same genotypes in 4,476 eQTL, which could not be 430 discovered using N2xCB4856 recombinant inbred lines. Alternatively, RIAlLs might have 431 less linkage disequilibrium between nearby variants and thus smaller eQTL regions of 432 interest than eQTL in wild C. elegans strains. The GWA eQTL in this study have a median 433 region of interest of $2.1 \mathrm{Mb}$ (ranged from $12 \mathrm{~kb}$ to $18 \mathrm{Mb}$ ), whereas the N2xCB4856 434 RIAILs eQTL showed a median size of $0.55 \mathrm{Mb}$ (ranged from $149 \mathrm{bp}$ to $6.8 \mathrm{Mb}$ ), which 435 might make the identification of underlying causal variants easier. We further found 17 436 distant eQTL hotspots overlapped between the two studies (Supplementary Fig. 9b). 437 However, these shared hotspots comprise different genes between the two studies, 438 indicating that variation in regulatory factors is not common between the linkage and 439 association mapping studies. Future research should leverage both types of mapping 440 studies to identify common regulatory mechanisms, focusing on local eQTL.

441 In addition to the high linkage disequilibrium across the $C$. elegans genome, the 442 recently discovered hyper-divergent genomic regions made this eQTL study challenging. 443 Approximately $20 \%$ of the genomes in some wild C. elegans strains were found to have 
444 extremely high diversity compared to the N2 reference genome ${ }^{27}$. Short-sequence reads

445 of wild C. elegans strains often fail to align to the N2 reference genome in these regions 446 and showed lower coverage than in other regions ${ }^{27}$. Similarly, expression levels of genes

447 in hyper-divergent regions could be underestimated because of the poor alignment of

448 RNA-seq reads. Therefore, we only used expression of transcripts in non-divergent 449 regions to map eQTL and flagged the loci that are in common hyper-divergent regions, 450 where we are less confident in the genotypes of wild strains (Supplementary Data 2). 451 Furthermore, we only used distant eQTL that are not in common hyper-divergent regions 452 to identify hotspots. Because hyper-divergent regions were suggested to be under long453 term balancing selection, our estimates of Tajima's $D$ in hotspots are probably biased 454 towards lower values. Future efforts using long-read sequencing are necessary to study 455 the sequence, expression, natural selection, and evolution of genes in hyper-divergent 456 regions.

457 Variation in gene expression was suggested to impact organism-level phenotypic 458 variation $^{7,64-66}$. Combining previous GWA studies in C. elegans with expression of genes 459 with eQTL, we used mediation analysis to search for organism-level phenotypic variation 460 that can be explained by variation in gene expression. Compared to previous studies 461 using mediation analysis on gene expression and eQTL data from the N2xCB4856 462 recombinant inbred lines ${ }^{9,43}$, we added a multiple testing correction procedure to our 463 mediation analysis. We performed mediation analysis on ABZ response variation ${ }^{44}$. The 464 causal gene ben-1 underlying the trait was identified using a burden mapping approach ${ }^{44}$ 465 along with prior knowledge ${ }^{67,68}$ about the role of beta-tubulin in this drug response. 466 Although two GWA QTL on chromosomes II and V were found, they were identified likely 467 because of their interchromosomal linkage disequilibrium to variants in the ben-1 locus ${ }^{44}$ 468 (Supplementary Fig. 8). The single-marker GWA mapping could not associate ABZ 469 response variation because of the extreme allelic heterogeneity at the ben-1 locus. 470 However, we used mediation analysis to identify ben-1 without consideration of prior 471 knowledge or burden mapping results, demonstrating the power of the approach (Fig. 472 4a). We further identified significant mediators for seven other organism-level traits (Fig. 
473 5). The expression of these mediator genes could affect the corresponding phenotypic 474 variation, which should be validated in the future.

475 Mediation analysis provides an efficient hypothesis-generating approach to be performed in parallel to fine mappings. Additionally, mediator genes could contribute to 477 organism-level phenotypic variation in addition to causal genes identified using fine 478 mappings. One limitation of fine mappings is that searching for causal genes and variants 479 is restricted to the QTL region of interest. Mediation analysis can make statistical 480 connections between the organism-level phenotypes and expression of genes far away 481 from the QTL. As mentioned above, large GWA QTL regions of interest make it difficult 482 to identify causal genes, which require validation using genome editing. Future $C$. 483 elegans GWA studies should use both fine mappings and mediation analysis to prioritize 484 candidate genes. If the candidate genes overlap between the two approaches, then 485 validation approaches can be initiated using genome editing. In cases where the two 486 approaches identify different candidate genes, prioritization using prior knowledge 487 across all genes identified by both approaches can inform which genes should be tested 488 for validation using genome editing. Previous studies using fine mappings prioritized 489 candidate genes harboring coding variants predicted to have strong functional impacts. 490 In mediation analysis, noncoding variants that likely affect expression of mediator genes 491 could also be nominated as candidates. For example, upstream variants were suggested 492 to underlie expression variation of the gene $s c b-1$, which mediated differences in 493 responses to bleomycin and three other chemotherapeutics ${ }^{9,69}$. To summarize, we 494 recommend using both fine mappings and mediation analyses to nominate candidate 495 genes and variants.

496 The goal of quantitative genetics is to understand the genetic basis and 497 mechanisms underlying phenotypic variation. Here, we showed that mediation analysis, 498 which uses expression and eQTL data to search connections between genetic variants 499 and complex traits, provides additional loci that might further explain phenotypic 500 variation. The framework we developed for mediation analysis complements marker501 based GWA mappings and is also applicable using various other intermediate traits, 502 such as small RNAs, proteins, and metabolites. Any genes and variants underlying 
503 variation in these factors can be nominated as candidates for phenotypic validation.

504 Furthermore, we could measure all of these data and complex traits from the exact same

505 samples using C. elegans, which can be easily grown at large scale to have synchronized

506 isogenic populations. Analyses using measurements of mRNAs, small RNAs, proteins,

507 and metabolites could strengthen conclusions about causal genes and mechanisms

508 underlying complex traits using a more holistic perspective of organismal phenotypic

509 variation. We foresee this strategy will greatly improve the powers of quantitative genetic

510 mappings in the future. 


\section{Methods}

\section{C. elegans strains}

513 We obtained 207 wild C. elegans strains from C. elegans Natural Diversity Resource $514(\mathrm{CeNDR})^{25}$. Animals were cultured at $20^{\circ} \mathrm{C}$ on modified nematode growth medium 515 (NGMA) containing 1\% agar and 0.7\% agarose to prevent burrowing and fed Escherichia 516 coli (E. coli) strain OP50 ${ }^{70}$. Prior to each assay, strains were grown for three generations 517 without starvation or encountering dauer-inducing conditions ${ }^{70}$.

\section{Animal growth and harvest}

520 We grew and harvested synchronized populations of each strain at the young adult stage

521 with independently grown and prepared biological replicates. Specifically, L4 larval stage 522 hermaphrodites were grown to the gravid adult stage on $6 \mathrm{~cm}$ plates and were bleached 523 to obtain synchronized embryos. Approximately 1,000 embryos were grown on each 10 $524 \mathrm{~cm}$ plate to the young adult stage and were harvested after the first embryo was 525 observed. M9 solution was used to wash harvested animals twice to remove E. coli. 526 Animals were then pelleted by centrifugation (2000 rpm for one minute) and Trizol 527 reagent (Ambion) was added to maintain RNA integrity before storage at $-80^{\circ} \mathrm{C}$.

\section{RNA extraction}

530 Frozen samples in Trizol were thawed at room temperature and $100 \mu \mathrm{L}$ acid-washed 531 sand (Sigma, catalog no. 274739) was added to help to disrupt animal tissues. Then 532 chloroform, isopropanol, and ethanol were used for phase separation, precipitation, and 533 washing steps, respectively. Total RNA pellets were resuspended in nuclease-free water.

534 The concentration of total RNA was determined using the Qubit RNA XR Assay Kit 535 (Invitrogen, catalog no. Q33224). RNA quality was measured using the 2100 Bioanalyzer 536 (Agilent). RNA samples with a minimum RNA integrity number (RIN) of 7 were used to 537 construct Illumina sequencing libraries. 
540 Illumina RNA-seq libraries were prepared in 96-well plates. Replicates of the same strain 541 were prepared in different 96-well plates. For each sample, mRNA was purified and 542 enriched from $1 \mu \mathrm{g}$ of total RNA using the NEBNext Poly(A) mRNA Magnetic Isolation 543 Module (New England Biolabs, catalog no. E7490L). RNA fragmentation, first and 544 second strand cDNA synthesis, and end-repair processing were performed with the 545 NEBNext Ultra II RNA Library Prep with Sample Purification Beads (New England 546 Biolabs, catalog no. E7775L). The cDNA libraries were adapter-ligated using adapters 547 and unique dual indexes in the NEBNext Multiplex Oligos for Illumina (New England 548 Biolabs, catalog no. E6440, E6442) and amplified using 12 PCR cycles. All procedures 549 were performed according to the manufacturer's protocols. The concentration of each 550 RNA-seq library was determined using Qubit dsDNA BR Assay Kit (Invitrogen, catalog 551 no. Q32853). Approximately 96 RNA-seq libraries were pooled and quantified with the 5522100 Bioanalyzer (Agilent) at Novogene, CA, USA. Each of the pools of libraries were 553 sequenced on a single lane of an Illumina NovaSeq 6000 platform, yielding 150-bp 554 paired-end (PE150) reads.

556 In total, RNA-seq data of 608 samples from 207 wild C. elegans strains in seven pooled 557 libraries were obtained with an average of 32.6 million reads per sample and a minimum 558 of 16.6 million reads. Of the 207 strains, 194 strains with three replicates and 13 strains 559 with two replicates.

Sequence processing and expression abundance quantification

562 Adapter sequences and low-quality reads in raw sequencing data were removed using 563 fastp (v0.20.0 $)^{71}$. FastQC (v0.11.8) analysis

564 (http://www.bioinformatics.babraham.ac.uk/projects/fastqc) was performed on trimmed 565 FASTQ files to assess read quality (adapter content, read-length distribution, per read 566 GC content, etc.). For RNA-seq mapping, SNV-substituted reference transcriptomes for 567 each of the wild C. elegans strains were generated using BCFtools (v.1.9) ${ }^{72}$, gffread $568(\mathrm{v} 0.11 .6)^{73}$, the N2 reference genome (WS276), a GTF file (WS276) ${ }^{53}$, and the hard-filtered 569 isotype variant call format (VCF) 20200815 CeNDR release (Supplementary Fig. 1). 
570 Transposable element (TE) consensus sequences of $C$. elegans were also extracted from

571 Dfam (release 3.3) ${ }^{74}$ using scripts (https://github.com/fansalon/TEconsensus). We used

572 Kallisto (v0.44.0) to (1) pseudoalign trimmed RNA-seq reads from each sample to the

573 transcriptome index built from the strain-specific SNV-substituted reference

574 transcriptome (65,173 transcripts) and TE consensus sequences (157 TEs) and (2)

575 quantify expression abundance at the transcript level ${ }^{75}$. On average, 31.3 million reads

576 pseudoaligned to the transcriptome index per sample with a minimum of 15.5 million

577 reads, which were sufficient to capture the expression of more than $70 \%$ of the $C$.

578 elegans reference genome genes. We used the 608 samples of 207 strains and 39,008

579 transcripts of protein-coding genes and pseudogenes in our analysis.

\section{Selection of reliably expressed transcripts}

582 We first normalized the raw counts of transcript expression abundances without the 583 default filtering of low abundance transcripts using the R package sleuth (v0.30.0 $)^{76}$. 584 Then, we filtered reliably expressed transcripts $(26,043)$ by requiring at least five 585 normalized counts in all the replicates of at least ten strains (Supplementary Fig. 1). We 586 also filtered out 3,775 transcripts of 2,597 genes that are in hyper-divergent genomic 587 regions of at least one strain. We further excluded 194 transcripts in hyper-divergent 588 regions of more than 107 of the 207 strains. In summary, we collected reliable expression 589 abundance for 25,849 transcripts of 16,094 genes (15,364 protein-coding genes and 730 590 pseudogenes).

\section{Selection of well clustered samples}

593 We used sample-to-sample distance to select well clustered samples (Supplementary

594 Fig. 1). We first summarized raw counts of reliably expressed transcripts into gene-level 595 abundances using the $\mathrm{R}$ package tximport $(\mathrm{v} 1.10 .1)^{77}$. Then, we performed variance 596 stabilizing transformations on the gene expression profile using the vst() function in the 597 R package DESeq2 (v1.26.0), which generated $\log _{2}$ scale normalized expression data ${ }^{78}$. 598 Sample-to-sample pairwise Euclidean distances among the 608 samples were 599 calculated using the generic function dist) in $R^{79}$. Our basic assumption is that intra- 
600 strain distances among replicates should be smaller than inter-strain distances. Because

601 the majority of the 207 wild C. elegans strains exhibit low overall genetic diversity (Fig.

$6021 d)^{24,35,80}$, we required that the intra-strain distances of replicates be smaller than the 603 median of inter-strain distances of the strain to other strains. Specifically, for each strain, 604 if all of its intra-strain distances were smaller than the median of its inter-strain distances, 605 then all of its replicates were kept. If none of its intra-strain distances were smaller than 606 the median of its inter-strain distances, then all samples of the strain were removed. For 607 strains with three replicates, if one or two of its three intra-strain distances were smaller 608 than the median of its inter-strain distances, then the two replicates with the minimum 609 distances were kept. After removal of some outlier samples, the median of inter-strain 610 distances would change. Therefore, we repeatedly performed the procedures of data 611 transformation, sample-to-sample distance calculation, and filtering by comparing inter612 and intra-strain distances until no more samples were removed. Eventually, 561 samples 613 of 207 strains were selected as well clustered samples, which comprised 147 strains 614 with three replicates and 60 strains with two replicates.

\section{Transcript expression abundance normalization}

617 We used the function norm_factors 0 in the R package sleuth $(\mathrm{v} 0.30 .0)^{76}$ to compute the 618 normalization factors for each sample using the raw transcripts per million reads (TPM) 619 of 22,268 reliably expressed transcripts in non-divergent regions of the 207 strains and 620 their well clustered samples. Then, we normalized the raw TPM of all the 25,849 reliably 621 expressed transcripts of each sample with the normalization factors and used $622 \log _{2}($ normalized TPM + 0.5) for downstream analysis unless indicated otherwise.

\section{Sample age estimation}

625 To further verify the homogeneous developmental stage of our samples, we evaluated 626 the age of each sample when they were harvested using the $\mathrm{R}$ package RAPToR $627(\mathrm{v} 1.1 .3)^{28}$ (Supplementary Fig. 1). As the requirement of the package, we first generated 628 gene-level expression abundances. Raw TPM of 22,268 reliably expressed transcripts in 629 non-divergent regions were summarized into abundances of 13,637 genes using the $R$ 
630 package tximport (v1.10.1) ${ }^{77}$. Normalization factors for each sample using gene-level

631 abundances were calculated as described for transcript level and were used to normalize

632 gene level TPM. Correlation of $\log _{2}($ normalized TPM + 0.5) of our data against the 633 reference gene expression time series (Cel_YA_2) in RAPToR was computed using the

634 function ae 0 in RAPToR with 10,489 intersected genes and default parameters.

Genetic and expression relatedness

637 Genetic variation data for 207 C. elegans isotypes were acquired from the hard-filtered 638 isotype variant call format (VCF) 20200815 CeNDR release. These variants were pruned 639 to the 851,105 biallelic single nucleotide variants (SNVs) without missing genotypes. We 640 converted this pruned VCF file to a PHYLIP file using the vcf2phylip.py script ${ }^{81}$. 641 Expression distance among the 207 wild strains was calculated based on the mean 642 expression of 22,268 transcripts without missing data using the function dist() in R. The 643 unrooted neighbor-joining trees for genetic and expression relatedness were made using 644 the R packages phangorn (v2.5.5) ${ }^{82}$, ape (v5.6) ${ }^{83}$ and ggtree (v1.14.6) ${ }^{84}$.

646 eQTL mapping

647 Input phenotype and genotype data

648 For the 25,849 transcripts, we summarized the expression abundance of replicates to 649 have the mean expression for each transcript of each strain as phenotypes used in GWA 650 mapping (Supplementary Data 1). Genotype data for each of the 207 strains were 651 acquired from the hard-filtered isotype VCF (20200815 CeNDR release).

Permutation-based FDR threshold

654 We performed GWA mapping using the pipeline cegwas2-nf 655 (https://github.com/AndersenLab/cegwas2-nf). The pipeline uses the eigen656 decomposition significance (EIGEN) threshold or the more stringent Bonferroni657 corrected significance (BF) threshold to correct for multiple testing because of the large 658 number of genetic markers (SNVs). To further correct for false positive QTL because of 659 the large number of transcript expression traits, we computed a permutation-based 
660 False Discovery Rate (FDR) at 5\%. We randomly selected 200 traits from our input 661 phenotype file and permuted each of them 200 times. These 40,000 permuted 662 phenotypes were used as input to call QTL using cegwas2-nf with EIGEN and BF 663 threshold, respectively, as previously described ${ }^{47,49,52}$. Briefly, we used $B C F t o o / s^{72}$ to filter 664 variants that had any missing genotype calls and variants that were below the $5 \%$ minor 665 allele frequency. Then, we used -indep-pairwise 50100.8 in PLINK v1.9 ${ }^{85,86}$ to prune the 666 genotypes to 20,991 markers with a linkage disequilibrium (LD) threshold of $r^{2}<0.8$ and 667 then generated the kinship matrix using the A.mat) function in the R package $r r B L U P$ $668(\mathrm{v} 4.6 .1)^{87}$. The number of independent tests $\left(N_{\text {test }}\right)$ within the genotype matrix was 669 estimated using the R package RSpectra (v0.16.0) (https://github.com/yixuan/RSpectra) 670 and correlateR (0.1) (https://github.com/AEBilgrau/correlateR). The eigen671 decomposition significance (EIGEN) threshold was calculated as $-\log _{10}\left(0.05 / N_{\text {test }}\right)$. We used the GWAS0 function in the rrBLUP package to perform the genome-wide mapping 673 with the EMMA algorithm ${ }^{32}$. QTL were defined by at least one marker that was above the 674 EIGEN or BF threshold. The EIGEN and BF \%5 FDR was calculated as the 95 percentile 675 of the significance of all the detected QTL under each threshold. The EIGEN and BF 5\% 676 FDR thresholds were 6.11 and 7.76, respectively.

678 eQTL mapping

679 We performed GWA mapping on the expression traits of the 25,849 transcripts as for 680 permuted expression traits but using the EIGEN 5\% FDR (6.11) as the threshold. We 681 identified QTL with significance that also passed the Bonferroni 5\% FDR threshold to 682 locate the best estimate of QTL positions with the highest significance. We used the 683 generic function corl in $\mathrm{R}$ and Pearson correlation coefficient to calculate the phenotypic 684 variance explained by each QTL. We used the $L D /$ function from the R package genetics 685 (v1.3.8.1.2) (https://cran.r-project.org/package=genetics) to calculate the LD correlation 686 coefficient $r^{2}$ among QTL for traits with multiple eQTL. 
689 Local eQTL were classified if the QTL was within a $2 \mathrm{Mb}$ region surrounding the

690 transcript. All other QTL were classified as distant.

\section{Heritability calculation}

693 Heritability estimates were calculated for each of the 25,849 traits used for eQTL 694 mapping as previously described ${ }^{88}$. Narrow-sense heritability $\left(h^{2}\right)$ was calculated with the 695 phenotype file and pruned genotypes in eQTL mapping using the functions mmer( and 696 pinl in the R package sommer (v4.1.2) $)^{89}$. Broad-sense heritability $\left(H^{2}\right)$ was calculated 697 using expression of replicates of each strain and the Imer function in the R package Ime4 (v1.1.21) with the model phenotype $\sim 1+(1 \mid \text { strain })^{90}$.

\section{Hotspot identification}

701 We first filtered out distant eQTL in common hyper-divergent genomic regions of wild C.

702 elegans strains. Common hyper-divergent regions were defined among our 206 strains 703 (reference N2 excluded) as described previously ${ }^{27}$. Briefly, we divided the genome into 1

$704 \mathrm{~kb}$ bins and calculated the percentage of 206 strains that are classified as hyper705 divergent in each bin. Common hyper-divergent regions were defined as bins $\geq 5 \%{ }^{27}$.

Distant eQTL hotspots were identified by dividing the genome into $0.5 \mathrm{cM}$ bins and counting the number of non-divergent distant eQTL that mapped to each bin. Significance was determined as bins with more eQTL than the 99th percentile of a

710 Poisson distribution using the maximum likelihood method and the function eqpois() in

711 the R package EnvStats ${ }^{1,3,9,91}$.

\section{Reanalysis of RIAILs eQTL}

714 We reclassified eQTL detected in a previous study using microarray expression data

715 from synchronized young adult populations of 208 recombinant inbred advanced 716 intercross lines (RIAILs) derived from N2 and CB4856,36. A total of 2,540 eQTL from

7172,196 probes were identified using linkage mappings ${ }^{9}$. We selected 2,387 eQTL of 2,054

718 probes that are in 2,003 live genes based on the probe-gene list in the $\mathrm{R}$ package 
719 linkagemapping (https://github.com/AndersenLab/linkagemapping) and the GTF file

720 (WS276) ${ }^{53}$. We classified 1,208 local eQTL and 1,179 distant eQTL as described above.

721 We further identified hotspots as above for 1,124 distant eQTL that are not in the hyper-

722 divergent regions of CB4856.

723

\section{Population genetics}

725 We use 851,105 biallelic SNVs with no missing calls among the 207 strains from the 726 hard-filtered VCF 20200815 CeNDR release to calculate population genomic statistics.

727 Tajima's D, Watterson's $\theta$, and pi were all calculated using scikit-alle ${ }^{92}$. Each of these 728 statistics was calculated for non-overlapping 1,000-bp windows across the genome.

\section{Fine mapping for causal genes underlying hotspots}

731 For transcript expression traits with distant eQTL in hotspots, we performed fine

732 mapping using the pipeline cegwas2-nf as previously described ${ }^{47}$. Briefly, we defined 733 QTL regions of interest from the GWA mapping as +/- 100 SNVs from the rightmost and 734 leftmost markers above the EIGEN 5\% FDR significance threshold. Then, using 735 genotype data from the imputed hard-filtered isotype VCF (20200815 CeNDR release), 736 we generated a QTL region of interest genotype matrix that was filtered as described 737 above, with the one exception that we did not perform LD pruning. We used PLINK $738 \mathrm{~V} 1.9^{85,86}$ to extract the LD between the markers used for fine mapping and the QTL peak 739 marker identified from GWA mappings. We used the same command as above to 740 perform fine mappings. To identify causal genes and variants that affect expression of 741 several transcripts underlying hotspots, we retained the fine-mapped candidate variants 742 that passed the following per trait filters: top 5\% most significant markers; out of 743 common hyper-divergent genomic regions; with negative BLOSUM ${ }^{93}$ scores as 744 characterized and annotated in $\mathrm{CeNDR}^{25}$.

\section{Enrichment analysis}


747 Gene set enrichment analyses were carried out for all genes found with transcript-level

748 eQTL and for genes with distant eQTL in each hotspot using the web-based tool

749 WormCat ${ }^{39}$.

750

751 Mediation analysis

752 GWA mapping of diverse C. elegans phenotypes

753 We obtained nine different phenotype data used in previous C.elegans natural variation

754 and GWA studies ${ }^{43,44,46-52}$. We filtered genetically distinct isotype strains for each trait 755 based on CeNDR (20200815 release) and performed GWA mapping as for permuted 756 expression traits but mostly using EIGEN or BF as the threshold according to the original 757 studies. GWA was performed under EIGEN for two studies originally using BF as the 758 threshold ${ }^{48,49}$.

761 For each QTL of the above phenotypes, we used the genotype (Exposure) at the 762 phenotype QTL peak, transcript expression traits (Mediator) that have eQTL overlapped 763 with the phenotype QTL, and the phenotype (Outcome) as input to perform mediation 764 analysis using the medTest/( function and 1,000 permutations for $p$-value correction in 765 the $\mathrm{R}$ package MultiMed (v2.6.0)

766 (https://bioconductor.org/packages/release/bioc/html/MultiMed.html). For mediation, 767 we used only strains with all of the three input data types available and where variation 768 was found. For instance, between the 202 strains used in the study of $A B Z$ resistance ${ }^{44}$ 769 and the 207 strains used in this study, 167 strains overlapped. Although we searched 770 overlapped eQTL against QTL in the GWA mapping for ABZ resistance using 202 strains 771 (Supplementary Fig. 8), 167 strains at maximum were used in mediation analysis. 772 Furthermore, because some transcripts were found in hyper-divergent regions in certain 773 strains and their expression data were filtered out, the rest of the strains with all of the 774 data types available might contain no variation in one or all of the three data types and 775 were not used in mediation analysis. For example, we found 1,193 eQTL overlapped with 
776 the QTL on chromosome II in the GWA mapping for ABZ resistance, but only 1,157

777 mediation analyses were performed.

778

779 For mediators with adjusted $p<0.05$ or mediation estimate greater than the $99^{\text {th }}$

780 percentile of the distribution of mediation estimates, we performed a second mediation

781 analysis as described previously ${ }^{9}$ using the mediate) function from the R package

782 mediation (version 4.5.0) ${ }^{94}$ to filter out the uninterpretable results where the proportion of

783 the total effect (the estimated effect of genotype on phenotype, ignoring expression) that

784 can be explained by the mediation effect (the estimated effect of expression on

785 phenotype) is negative or larger than $100 \%$.

786

787 GWA of traits regressed by transcript expression

788 We regressed the trait animal length (q90.TOF) ${ }^{44}$ by expression of every transcript using

789 the generic function residuals $[$ in $\mathrm{R}$, which fits a linear model with the formula (phenotype

790 expression) to account for any differences in phenotype parameters present in

791 transcript expression. Then GWA was performed for each regressed trait as for permuted

792 expression traits using BF as the threshold. 


\section{Acknowledgements}

797 We would like to thank Stefan Zdraljevic and Samuel J. Widmayer for helpful

798 suggestions. G.Z. is supported by the NSF-Simons Center for Quantitative Biology at 799 Northwestern University (awards Simons Foundation/SFARI 597491-RWC and the 800 National Science Foundation 1764421). S.R.H. was funded by a DFG fellowship (HA 801 8449/1-1) from the Deutsche Forschungsgemeinschaft (www.dfg.de). E.C.A. is 802 supported by a National Science Foundation CAREER Award (IOS-1751035) and a grant 803 from the National Institutes of Health R01 DK115690. The C. elegans Natural Diversity 804 Resource is supported by a National Science Foundation Living Collections Award to 805 R.E.T. and E.C.A. (1930382). We also like to thank WormBase for which these analyses 806 would not have been possible.

\section{Author contributions}

808 E.C.A. conceived of and designed the study. D.L., S.R.H., and G.Z. prepared C. elegans 809 cultures. G.Z. and N.M.R. performed RNA-seq experiments. G.Z. analyzed the data. G.Z. 810 and E.C.A. wrote the manuscript.

811 Competing interests

812 The authors declare no competing interests.

\section{Data and code availability}

814 The raw RNA-seq data generated in this study are available at the NCBI Sequence Read 815 Archive (Project PRJNA669810). The raw expression counts and TPM quantified in this 816 study are available at NCBI's Gene Expression Omnibus (Series GSE186719). The RNA817 seq mapping pipeline can be found at https://github.com/AndersenLab/PEmRNA-seq818 nf. The mediation analysis pipeline can be found at 819 https://github.com/AndersenLab/mediation GWAeQTL. The datasets and code for 820 generating all figures can be found at https://github.com/AndersenLab/WI-Ce-eQTL. 


\section{References}

823 1. Brem, R. B., Yvert, G., Clinton, R. \& Kruglyak, L. Genetic dissection of

824 transcriptional regulation in budding yeast. Science 296, 752-755 (2002).

825 2. West, M. A. L. et al. Global eQTL mapping reveals the complex genetic

826 architecture of transcript-level variation in Arabidopsis. Genetics 175, 1441-1450

827 (2007).

828 3. Rockman, M. V., Skrovanek, S. S. \& Kruglyak, L. Selection at linked sites shapes heritable phenotypic variation in C. elegans. Science 330, 372-376 (2010).

4. Zan, Y., Shen, X., Forsberg, S. K. G. \& Carlborg, Ö. Genetic Regulation of Transcriptional Variation in Natural Arabidopsis thaliana Accessions. G3 6, 23192328 (2016).

5. Kita, R., Venkataram, S., Zhou, Y. \& Fraser, H. B. High-resolution mapping of cisregulatory variation in budding yeast. Proc. Natl. Acad. Sci. U. S. A. 114, E10736E10744 (2017).

6. GTEx Consortium et al. Genetic effects on gene expression across human tissues. Nature 550, 204-213 (2017).

7. Albert, F. W., Bloom, J. S., Siegel, J., Day, L. \& Kruglyak, L. Genetics of transregulatory variation in gene expression. Elife 7, 1-39 (2018).

8. GTEx Consortium. The GTEx Consortium atlas of genetic regulatory effects across human tissues. Science 369, 1318-1330 (2020).

9. Evans, K. S. \& Andersen, E. C. The Gene scb-1 Underlies Variation in Caenorhabditis elegans Chemotherapeutic Responses. G3 10, 2353-2364 (2020).

10. Rockman, M. V. \& Kruglyak, L. Genetics of global gene expression. Nat. Rev. Genet. 7, 862-872 (2006).

11. Albert, F. W. \& Kruglyak, L. The role of regulatory variation in complex traits and disease. Nat. Rev. Genet. 16, 197-212 (2015). (2021). 
13. Brem, R. B., Yvert, G., Clinton, R. \& Kruglyak, L. Genetic dissection of transcriptional regulation in budding yeast. Science 296, 752-755 (2002).

14. Gaertner, B. E. \& Phillips, P. C. Caenorhabditis elegans as a platform for molecular quantitative genetics and the systems biology of natural variation. Genet. Res. 92 , 331-348 (2010).

15. Snoek, B. L. et al. WormQTL2: an interactive platform for systems genetics in Caenorhabditis elegans. Database 2020, (2020).

16. Evans, K. S., van Wijk, M. H., McGrath, P. T., Andersen, E. C. \& Sterken, M. G. From QTL to gene: C. elegans facilitates discoveries of the genetic mechanisms underlying natural variation. Trends Genet. 0, (2021).

17. Li, Y. et al. Mapping determinants of gene expression plasticity by genetical genomics in C. elegans. PLoS Genet. 2, e222 (2006).

18. Viñuela, A., Snoek, L. B., Riksen, J. A. G. \& Kammenga, J. E. Genome-wide gene expression regulation as a function of genotype and age in C. elegans. Genome Res. 20, 929-937 (2010).

19. Li, Y. et al. Global genetic robustness of the alternative splicing machinery in Caenorhabditis elegans. Genetics 186, 405-410 (2010).

20. Sterken, M. G. et al. Ras/MAPK Modifier Loci Revealed by eQTL in Caenorhabditis elegans. G3 7, 3185-3193 (2017).

874 23. Snoek, B. L. et al. The genetics of gene expression in a Caenorhabditis elegans multiparental recombinant inbred line population. G3 11, (2021).

24. Andersen, E. C. et al. Chromosome-scale selective sweeps shape Caenorhabditis

878 25. Cook, D. E., Zdraljevic, S., Roberts, J. P. \& Andersen, E. C. CeNDR, the 879 Caenorhabditis elegans natural diversity resource. Nucleic Acids Res. 45, D650880 D657 (2017). 
26. Thompson, O. A. et al. Remarkably Divergent Regions Punctuate the Genome Assembly of the Caenorhabditis elegans Hawaiian Strain CB4856. Genetics 200, 975-989 (2015).

27. Lee, D. et al. Balancing selection maintains hyper-divergent haplotypes in Caenorhabditis elegans. Nat Ecol Evol 1-14 (2021).

28. Bulteau, R. \& Francesconi, M. Real age prediction from the transcriptome with RAPToR. bioRxiv 2021.09.07.459270 (2021) doi:10.1101/2021.09.07.459270.

29. Crombie, T. A. et al. Deep sampling of Hawaiian Caenorhabditis elegans reveals high genetic diversity and admixture with global populations. Elife 8, e50465 (2019).

30. Bloom, J. S., Ehrenreich, I. M., Loo, W. T., Lite, T.-L. V. \& Kruglyak, L. Finding the sources of missing heritability in a yeast cross. Nature 494, 234-237 (2013).

31. Chen, A., Liu, Y., Williams, S. M., Morris, N. \& Buchner, D. A. Widespread epistasis regulates glucose homeostasis and gene expression. PLoS Genet. 13, e1007025 (2017).

32. Kang, H. M. et al. Efficient control of population structure in model organism association mapping. Genetics 178, 1709-1723 (2008).

33. Li, J. \& Ji, L. Adjusting multiple testing in multilocus analyses using the eigenvalues of a correlation matrix. Heredity 95, 221-227 (2005).

34. Campos, T. L., Korhonen, P. K., Sternberg, P. W., Gasser, R. B. \& Young, N. D.

35. Cutter, A. D. Nucleotide polymorphism and linkage disequilibrium in wild populations of the partial selfer Caenorhabditis elegans. Genetics 172, 171-184 (2006).

36. Rockman, M. V. \& Kruglyak, L. Recombinational landscape and population genomics of Caenorhabditis elegans. PLoS Genet. 5, e1000419 (2009). spatiotemporal resolution. Nature 512, 400-405 (2014). 
Hundreds of Drosophila and Caenorhabditis elegans Transcription Factors. Genetics 208, 937-949 (2018).

39. Holdorf, A. D. et al. WormCat: An Online Tool for Annotation and Visualization of Caenorhabditis elegans Genome-Scale Data. Genetics 214, 279-294 (2020).

40. Satterlee, J. S. et al. Specification of thermosensory neuron fate in C. elegans requires ttx-1, a homolog of otd/Otx. Neuron 31, 943-956 (2001).

41. Kagoshima, H. \& Kohara, Y. Co-expression of the transcription factors $\mathrm{CEH}-14$ and TTX-1 regulates AFD neuron-specific genes gcy-8 and gcy-18 in C. elegans. Dev. Biol. 399, 325-336 (2015).

42. Fairfax, B. P. et al. Innate immune activity conditions the effect of regulatory variants upon monocyte gene expression. Science 343, 1246949 (2014).

43. Evans, K. S. et al. Natural variation in the sequestosome-related gene, sqst-5, underlies zinc homeostasis in Caenorhabditis elegans. PLoS Genet. 16, e1008986 (2020).

44. Hahnel, S. R. et al. Extreme allelic heterogeneity at a Caenorhabditis elegans betatubulin locus explains natural resistance to benzimidazoles. PLoS Pathog. 14, e1007226 (2018).

45. Churchill, G. A., Gatti, D. M., Munger, S. C. \& Svenson, K. L. The Diversity Outbred mouse population. Mamm. Genome 23, 713-718 (2012).

46. Cook, D. E. et al. The Genetic Basis of Natural Variation in Caenorhabditis elegans Telomere Length. Genetics 204, 371-383 (2016).

47. Zdraljevic, S. et al. Natural variation in C. elegans arsenic toxicity is explained by differences in branched chain amino acid metabolism. Elife 8, e40260 (2019).

48. Zdraljevic, S. et al. Natural variation in a single amino acid substitution underlies physiological responses to topoisomerase II poisons. PLoS Genet. 13, e1006891 (2017).

937 49. Na, H., Zdraljevic, S., Tanny, R. E., Walhout, A. J. M. \& Andersen, E. C. Natural variation in a glucuronosyltransferase modulates propionate sensitivity in a $\mathrm{C}$.

940 50. Evans, K. S. et al. Two novel loci underlie natural differences in Caenorhabditis 
elegans abamectin responses. PLoS Pathog. 17, e1009297 (2021).

942 51. Lee, D. et al. Selection and gene flow shape niche-associated variation in

943 pheromone response. Nat Ecol Evol 3, 1455-1463 (2019).

944 52. Zhang, G., Mostad, J. D. \& Andersen, E. C. Natural variation in fecundity is

$945 \quad$ correlated with species-wide levels of divergence in Caenorhabditis elegans. G3 (2021) doi:10.1093/g3journal/jkab168.

53. Harris, T. W. et al. WormBase: a modern Model Organism Information Resource. Nucleic Acids Res. 48, D762-D767 (2020).

54. Thyagarajan, B. et al. ETS-4 is a transcriptional regulator of life span in Caenorhabditis elegans. PLoS Genet. 6, e1001125 (2010).

55. Petersen, C., Dirksen, P. \& Schulenburg, H. Why we need more ecology for genetic models such as C. elegans. Trends Genet. 31, 120-127 (2015).

56. Sterken, M. G., Snoek, L. B., Kammenga, J. E. \& Andersen, E. C. The laboratory domestication of Caenorhabditis elegans. Trends Genet. 31, 224-231 (2015).

57. Frézal, L. \& Félix, M.-A. The natural history of model organisms: C. elegans outside the Petri dish. Elife 4, e05849 (2015).

58. Schulenburg, H. \& Félix, M.-A. The Natural Biotic Environment of Caenorhabditis elegans. Genetics 206, 55-86 (2017).

59. Crombie, T. A. et al. Local adaptation and spatiotemporal patterns of genetic

60. Thomas, J. H. Adaptive evolution in two large families of ubiquitin-ligase adapters in nematodes and plants. Genome Res. 16, 1017-1030 (2006). diversity revealed by repeated sampling of Caenorhabditis elegans across the Hawaiian Islands. bioRxiv 2021.10.11.463952 (2021)

61. de Nadal, E., Ammerer, G. \& Posas, F. Controlling gene expression in response to stress. Nat. Rev. Genet. 12, 833-845 (2011).

62. Denver, D. R. et al. The transcriptional consequences of mutation and natural selection in Caenorhabditis elegans. Nat. Genet. 37, 544-548 (2005).

63. Rifkin, S. A., Houle, D., Kim, J. \& White, K. P. A mutation accumulation assay reveals a broad capacity for rapid evolution of gene expression. Nature 438, 220- 
223 (2005).

972 64. King, M. C. \& Wilson, A. C. Evolution at two levels in humans and chimpanzees.

973 Science 188, 107-116 (1975).

974 65. Oliver, F. et al. Regulatory variation at glypican-3 underlies a major growth QTL in

975 mice. PLoS Biol. 3, e135 (2005).

66. Courtier-Orgogozo, V., Arnoult, L., Prigent, S. R., Wiltgen, S. \& Martin, A. Gephebase, a database of genotype-phenotype relationships for natural and domesticated variation in Eukaryotes. Nucleic Acids Res. 48, D696-D703 (2020).

67. Driscoll, M., Dean, E., Reilly, E., Bergholz, E. \& Chalfie, M. Genetic and molecular analysis of a Caenorhabditis elegans beta-tubulin that conveys benzimidazole sensitivity. J. Cell Biol. 109, 2993-3003 (1989).

68. Kwa, M. S., Veenstra, J. G. \& Roos, M. H. Benzimidazole resistance in Haemonchus contortus is correlated with a conserved mutation at amino acid 200 in beta-tubulin isotype 1. Mol. Biochem. Parasitol. 63, 299-303 (1994).

69. Brady, S. C. et al. A Novel Gene Underlies Bleomycin-Response Variation in Caenorhabditis elegans. Genetics 212, 1453-1468 (2019).

70. Andersen, E. C., Bloom, J. S., Gerke, J. P. \& Kruglyak, L. A variant in the neuropeptide receptor npr-1 is a major determinant of Caenorhabditis elegans growth and physiology. PLoS Genet. 10, e1004156 (2014).

71. Chen, S., Zhou, Y., Chen, Y. \& Gu, J. fastp: an ultra-fast all-in-one FASTQ preprocessor. Bioinformatics 34, i884-i890 (2018).

72. Li, H. A statistical framework for SNP calling, mutation discovery, association mapping and population genetical parameter estimation from sequencing data. Bioinformatics 27, 2987-2993 (2011).

73. Pertea, G. \& Pertea, M. GFF Utilities: GffRead and GffCompare. F1000Res. 9, 304 (2020).

74. Storer, J., Hubley, R., Rosen, J., Wheeler, T. J. \& Smit, A. F. The Dfam community resource of transposable element families, sequence models, and genome annotations. Mob. DNA 12, 2 (2021).

75. Bray, N. L., Pimentel, H., Melsted, P. \& Pachter, L. Near-optimal probabilistic RNA- 
seq quantification. Nat. Biotechnol. 34, 525-527 (2016).

76. Pimentel, H., Bray, N. L., Puente, S., Melsted, P. \& Pachter, L. Differential analysis of RNA-seq incorporating quantification uncertainty. Nat. Methods 14, 687-690 (2017).

77. Soneson, C., Love, M. I. \& Robinson, M. D. Differential analyses for RNA-seq: transcript-level estimates improve gene-level inferences. F1000Res. 4, 1521 (2015).

78. Love, M. I., Huber, W. \& Anders, S. Moderated estimation of fold change and dispersion for RNA-seq data with DESeq2. Genome Biol. 15, 550 (2014).

79. Core Team, R. \& Others. R: A language and environment for statistical computing. Vienna, Austria: R Foundation for Statistical Computing. Available (2013).

80. Barrière, A. \& Félix, M.-A. Natural variation and population genetics of Caenorhabditis elegans. WormBook 1-19 (2005).

81. Ortiz, E. M. vcf2phylip v2.0: convert a VCF matrix into several matrix formats for phylogenetic analysis. (2019). doi:10.5281/zenodo.2540861.

82. Schliep, K. P. phangorn: phylogenetic analysis in R. Bioinformatics 27, 592-593 (2011).

83. Paradis, E. \& Schliep, K. ape 5.0: an environment for modern phylogenetics and evolutionary analyses in R. Bioinformatics 35, 526-528 (2019).

84. Yu, G., Smith, D. K., Zhu, H., Guan, Y. \& Lam, T. T. Ggtree : An r package for visualization and annotation of phylogenetic trees with their covariates and other associated data. Methods Ecol. Evol. 8, 28-36 (2017).

85. Purcell, S. et al. PLINK: a tool set for whole-genome association and populationbased linkage analyses. Am. J. Hum. Genet. 81, 559-575 (2007).

86. Chang, C. C. et al. Second-generation PLINK: rising to the challenge of larger and richer datasets. Gigascience 4, 7 (2015).

87. Endelman, J. B. Ridge regression and other kernels for genomic selection with $\mathrm{R}$ package rrBLUP. Plant Genome 4, 250-255 (2011).

88. Wit, J., Rodriguez, B. C. \& Andersen, E. C. Natural variation in Caenorhabditis elegans responses to the anthelmintic emodepside. Int. J. Parasitol. Drugs Drug 
Resist. 16, 1-8 (2021).

89. Covarrubias-Pazaran, G. Genome-Assisted Prediction of Quantitative Traits Using the R Package sommer. PLoS One 11, e0156744 (2016).

1034 90. Bates, D., Mächler, M., Bolker, B. \& Walker, S. Fitting Linear Mixed-Effects Models 1035 Using Ime4. Journal of Statistical Software, Articles 67, 1-48 (2015).

1036 91. Millard, S. P. EnvStats: An R Package for Environmental Statistics. (Springer-Verlag 1037 New York, 2013).

1038 92. Miles, A., Ralph, P., Rae, S. \& Pisupati, R. cggh/scikit-allel: v1.2.1. (2019).

1039 doi:10.5281/zenodo.3238280.

1040 93. Henikoff, S. \& Henikoff, J. G. Amino acid substitution matrices from protein blocks.

1041 Proc. Natl. Acad. Sci. U. S. A. 89, 10915-10919 (1992).

1042 94. Tingley, D., Yamamoto, T., Hirose, K., Keele, L. \& Imai, K. mediation: R Package 1043 for Causal Mediation Analysis. Journal of Statistical Software, Articles 59, 1-38 1044 (2014). 
Supplementary Information

4 An atlas of gene expression variation across the Caenorhabditis elegans species 5

6 Gaotian Zhang ${ }^{1}$, Nicole M. Roberto ${ }^{1}$, Daehan Lee ${ }^{1}$, Steffen R. Hahnel ${ }^{1}$, and Erik C.

7 Andersen ${ }^{1, *}$

8

9 1. Department of Molecular Biosciences, Northwestern University, Evanston, IL 60208, 10 USA 
18 File Name: Supplementary Data 1

19 Description: Expression abundances and heritabilities of 25,849 transcripts in 207 wild

20 C. elegans strains

22 File Name: Supplementary Data 2

23 Description: GWA mapping results of 5,291 transcript expression traits, eQTL 24 classification, and distant eQTL hotspots

26 File Name: Supplementary Data 3

27 Description: Enrichment of genes with eQTL, distant eQTL in hotspots

29 File Name: Supplementary Data 4

30 Description: Fine mappings for distant eQTL in hotspots 


\section{$31 \quad$ Supplementary Figures}

\section{Workflow of RNA-seq data analysis}

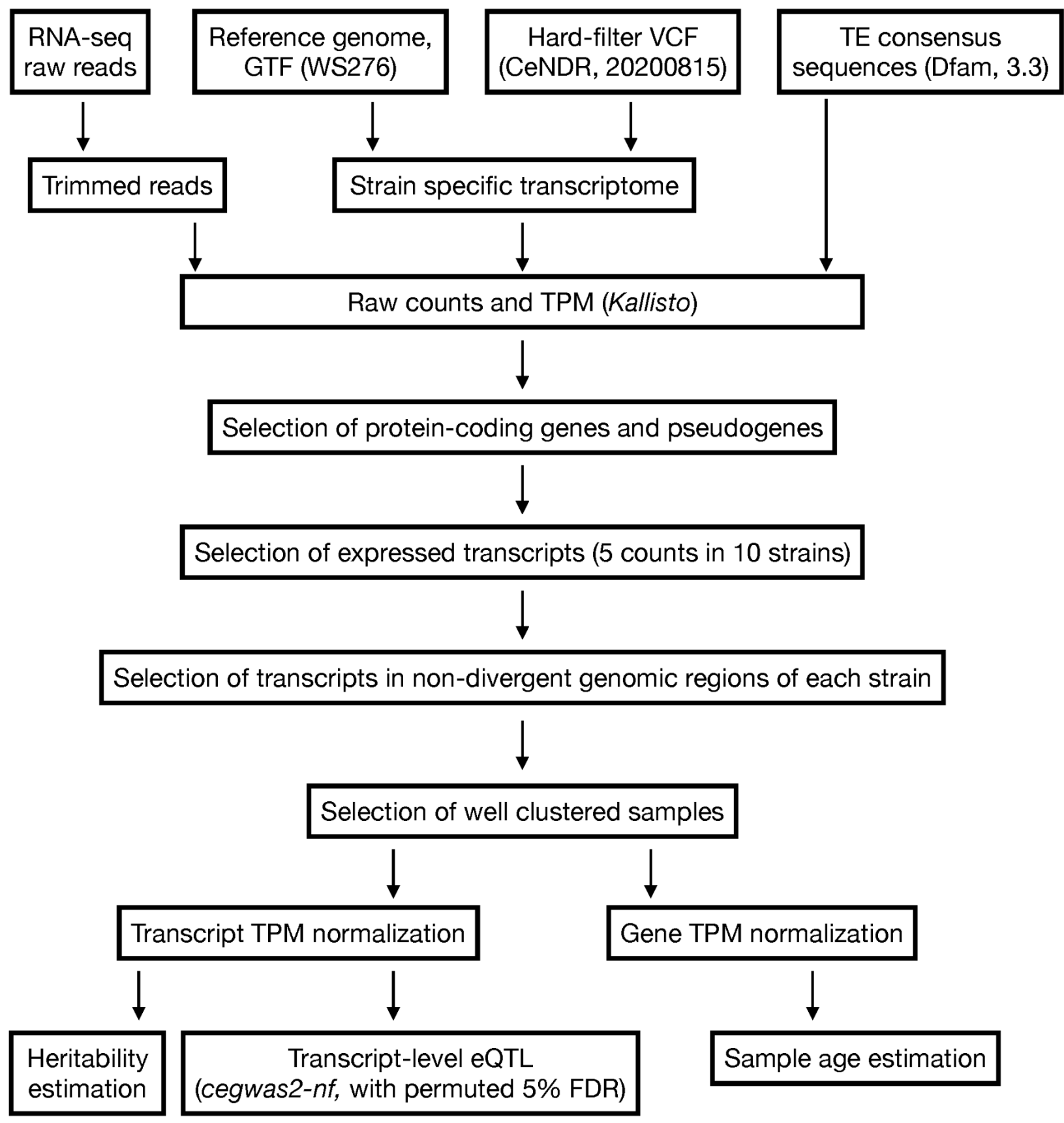

\section{Supplementary Fig. 1}

34 Workflow of RNA-seq data processing. 


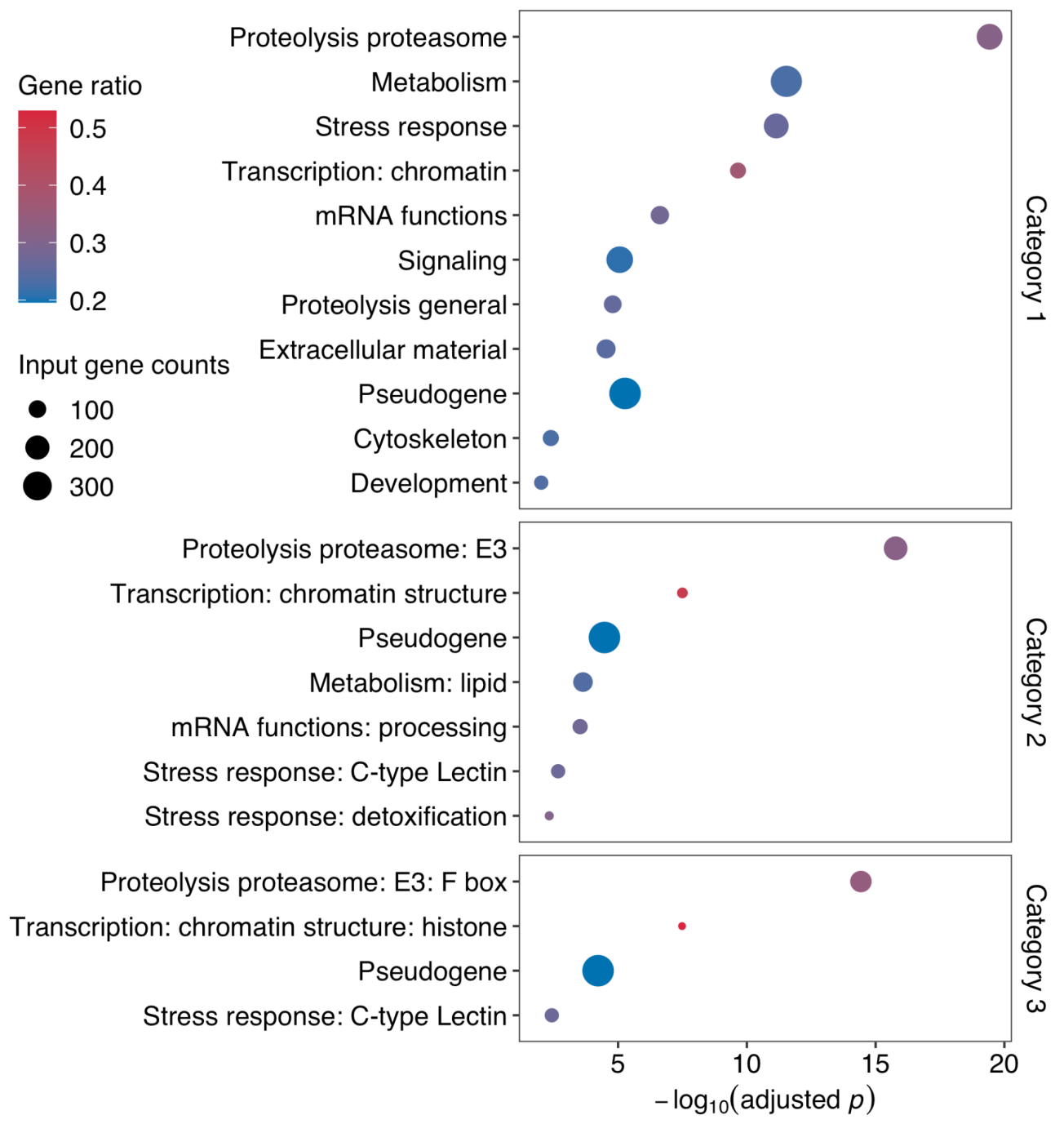

\section{Supplementary Fig. 2}

39 Gene set enrichment analysis for genes with transcript level eQTL. Enriched gene 40 classes of broad and specific categories (Category 1 to 3$)^{39}$ are shown on the y axis. 41 Bonferroni FDR corrected significance values using Fisher Exact Test for gene set 42 enrichment analysis are shown on the $x$ axis. The sizes of the circles correspond to the 43 input gene counts of the annotation and the colors of the circles correspond to the gene 44 ratio of input gene counts to total gene counts of the annotation. 


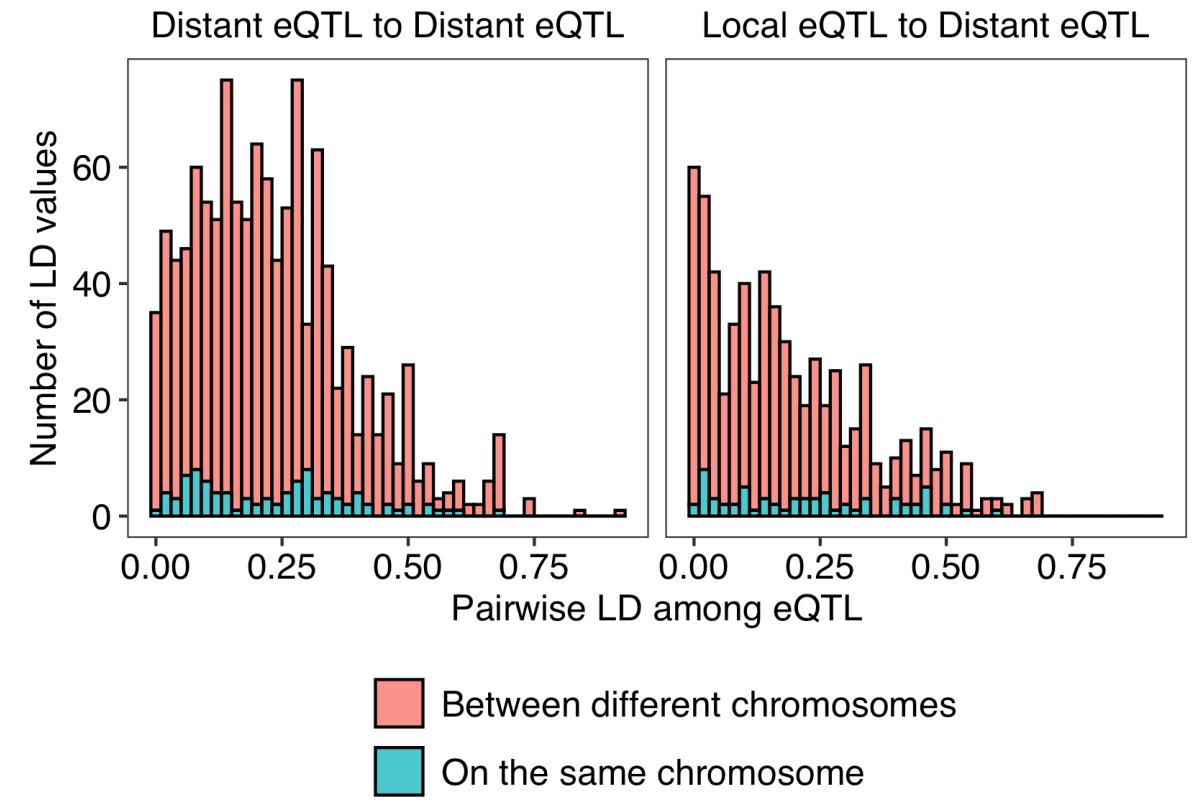

\section{Supplementary Fig. 3}

47 A histogram showing the distribution of linkage disequilibrium (LD) values ( $x$-axis) among 48 QTL of multiple eQTL of transcript expression traits. A total of 861 traits were found with 49 multiple eQTL. LD of eQTL from the same chromosome and different chromosomes are 50 colored salmon and blue, respectively. 

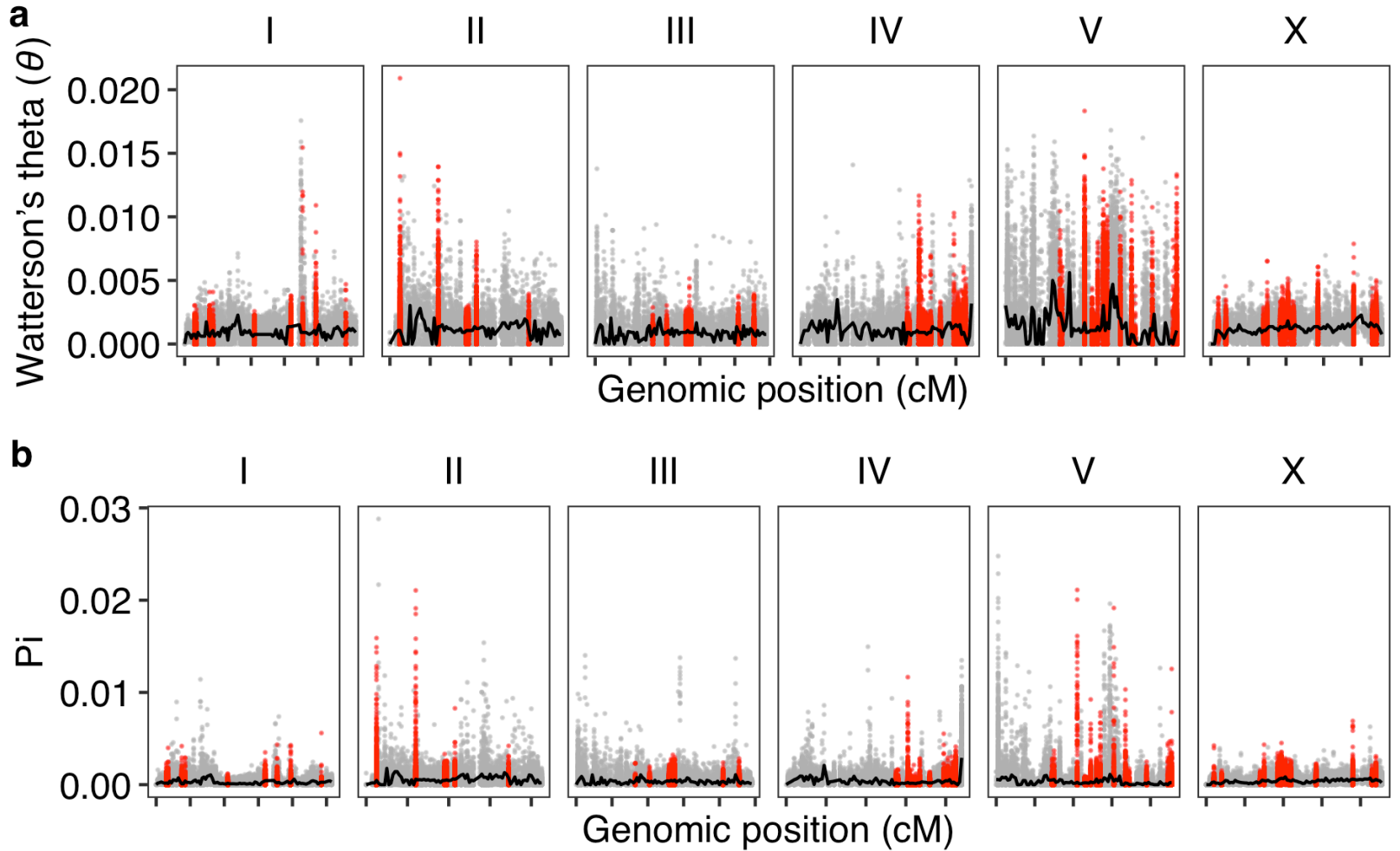

C
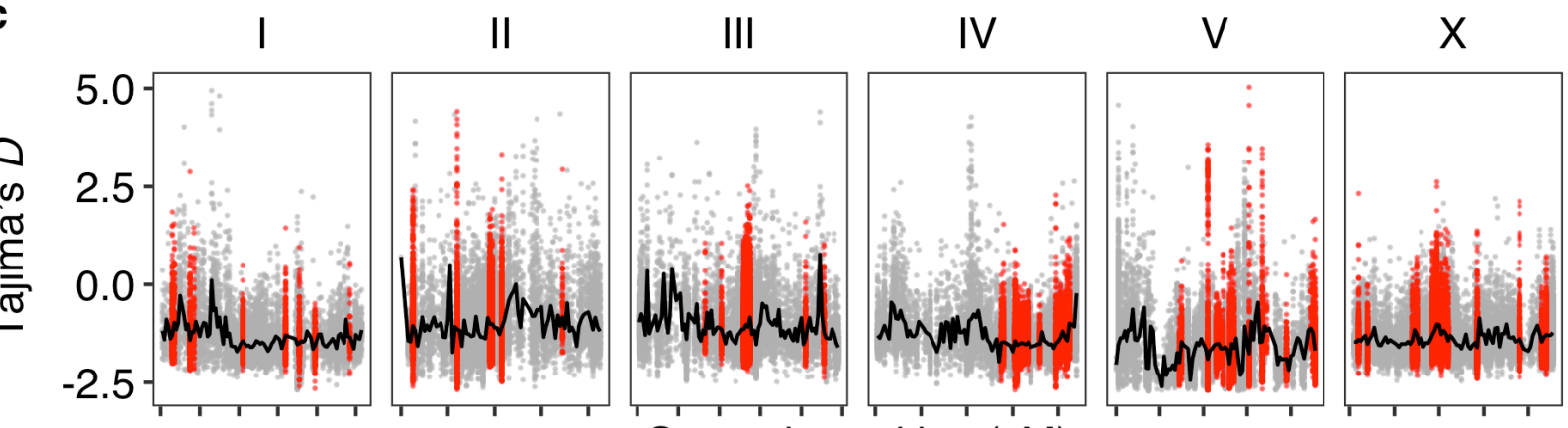

Genomic position (cM)

\section{Supplementary Fig. 4}

54 Genome-wide pattern of (a) Watterson's theta $(\theta)$, (b) nucleotide diversity (pi), and (c) Tajima's $D$. Each point represents the value (y-axis) for a non-overlapping $1 \mathrm{~kb}$ genomic window and is plotted against the genome position (x-axis) with tick marks denoting every $10 \mathrm{cM}$. Points for genomic windows in distant eQTL hotspots are colored red. Other points are colored gray. Median values of each statistic in each $0.5 \mathrm{cM}$ bin were colored black. Tajima's D values that suggest purifying selection are outliers for most

60 values within a hotspot.

61 
bioRxiv preprint doi: https://doi.org/10.1101/2022.02.06.479320; this version posted February 9, 2022. The copyright holder for this preprint (which was not certified by peer review) is the author/funder, who has granted bioRxiv a license to display the preprint in perpetuity. It is made available under aCC-BY 4.0 International license.

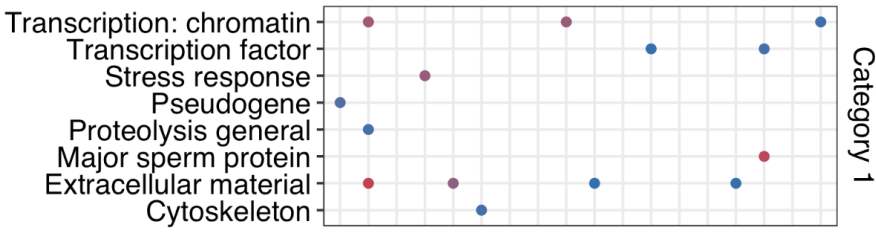

Transmembrane transport: lipid Transcription: chromatin structure Transcription: chromatin modification Transcription factor: homeodomain Stress response: heat Stress response: detoxification Signaling: small GTPase Signaling: hedgehog-like Proteolysis proteasome: E3 Proteolysis general: metallopeptidaseNon-coding RNA: small RNA Metabolism: mitochondria Major sperm protein Extracellular material: collagen Development: somatic Cytoskeleton: other Cytoskeleton: cadherin

Transmembrane transport: lipid Transcription: chromatin structure: histoneTranscription: chromatin modification: other

Transcription factor: homeodomain Stress response: heat Stress response: detoxification: CYP Signaling: hedgehog-like Proteolysis proteasome: E3: $F$ box Proteolysis general: metallopeptidase Non-coding RNA: small RNA: other mRNA functions: processing: helicase Metabolism: lipid: binding Major sperm protein Extracellular material: collagen Development: somatic Cytoskeleton: other Cytoskeleton: cadherin

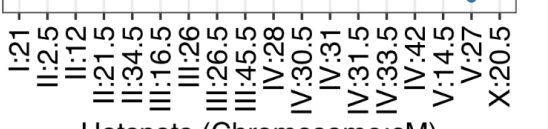

Hotspots (Chromosome:cM)

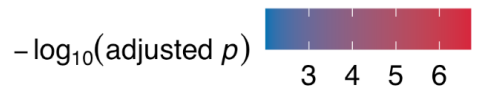

\section{Supplementary Fig. 5}

65 Gene set enrichment analysis for genes with transcript level distant eQTL in each 66 hotspot. Broad and specific categories of enriched gene (Category 1 to 3 ) ) $^{39}$ are shown 67 on the y axis. Distant eQTL hotspots with significant gene set enrichment are shown on 68 the $x$ axis. The colors of the circles correspond to Bonferroni FDR corrected significance 69 values using Fisher Exact Test. 


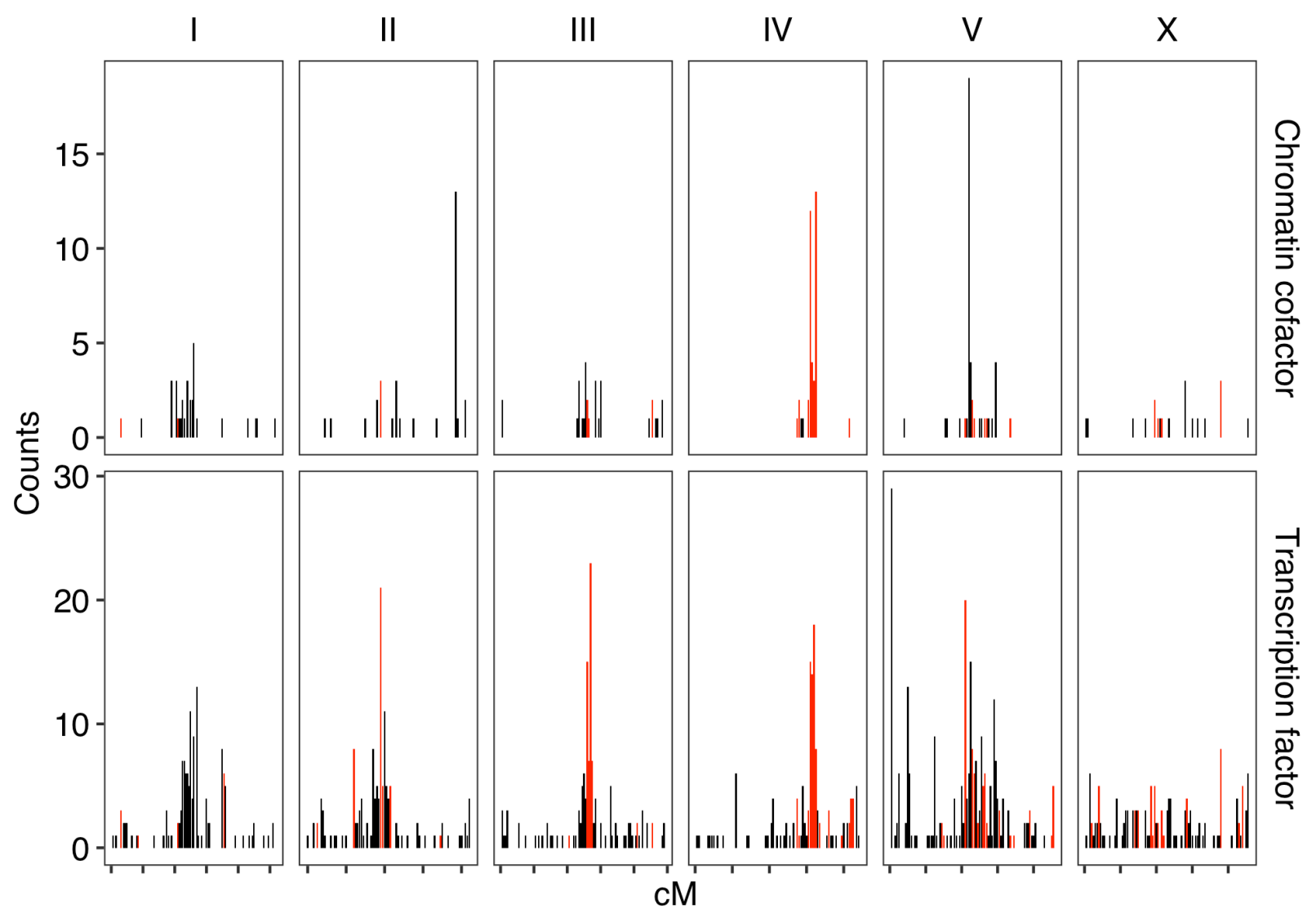

\section{Supplementary Fig. 6}

72 Number of genes encoding chromatin cofactors and transcription factors in each $0.5 \mathrm{cM}$

73 bin of the $C$. elegans genome. Bins that were identified as distant eQTL hotspots are 74 colored red. Other bins are colored black. 

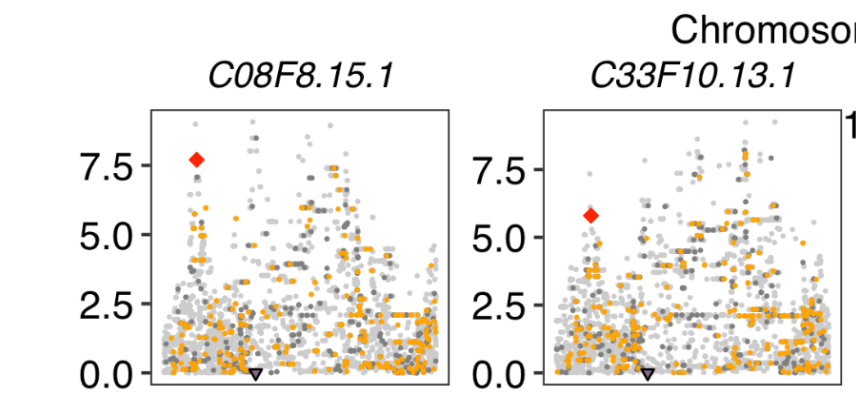

Chromosome IV: $31 \mathrm{cM}$
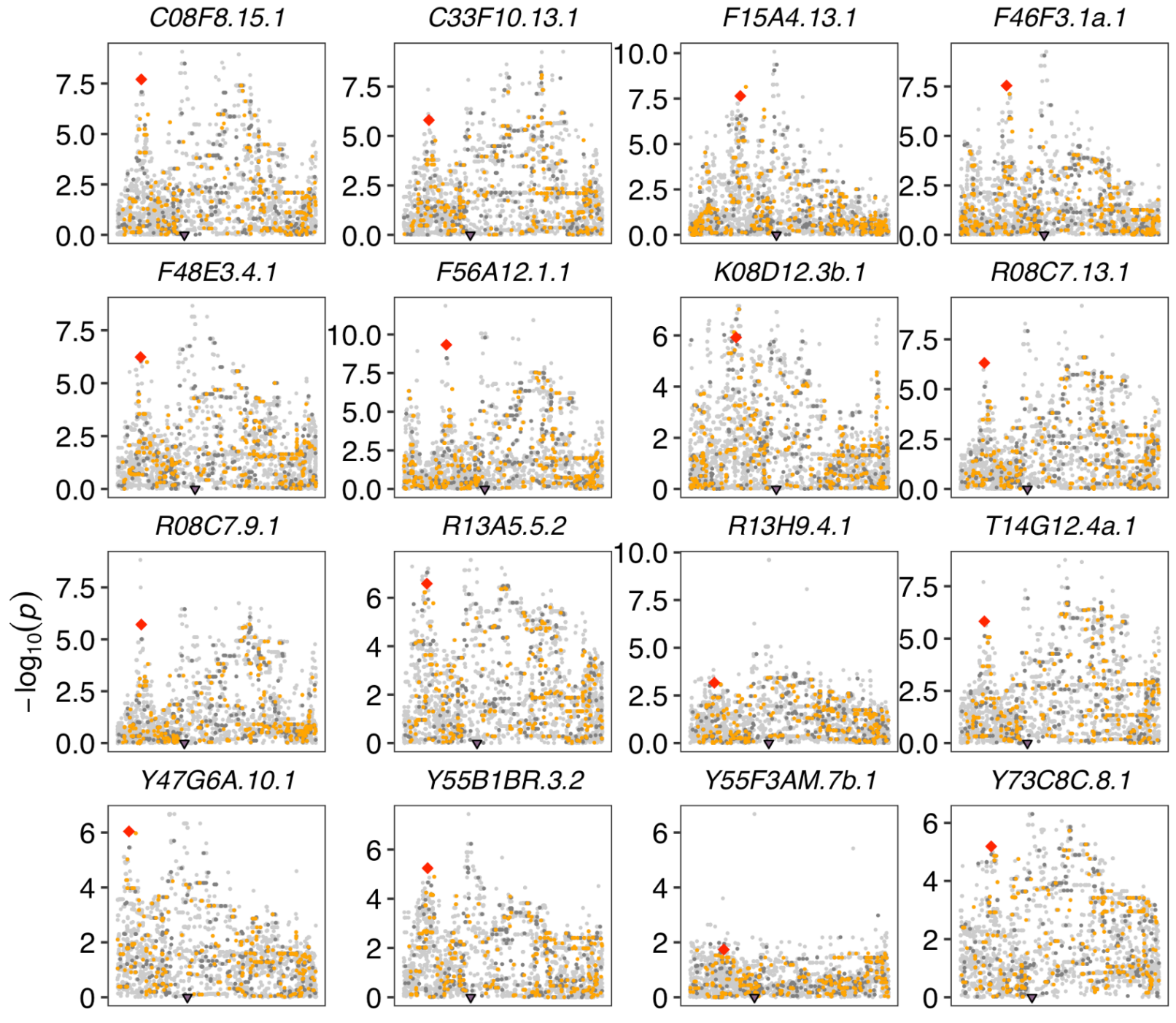

T14G12.4a.1

ZK993.1a.1

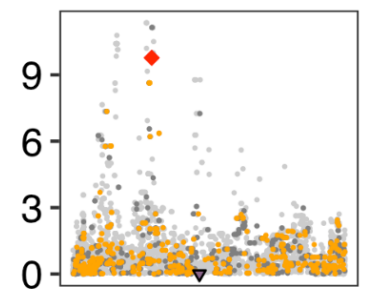

Genomic position (Mb)

Candidate genes $\bullet$ hil-2 
77

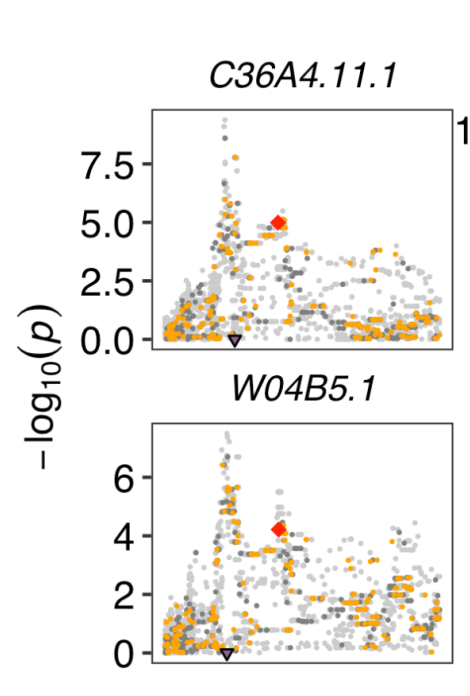

Chromosome I: $21 \mathrm{cM}$

F19G12.9a.1

K02E2.6.1

W04A4.2.1
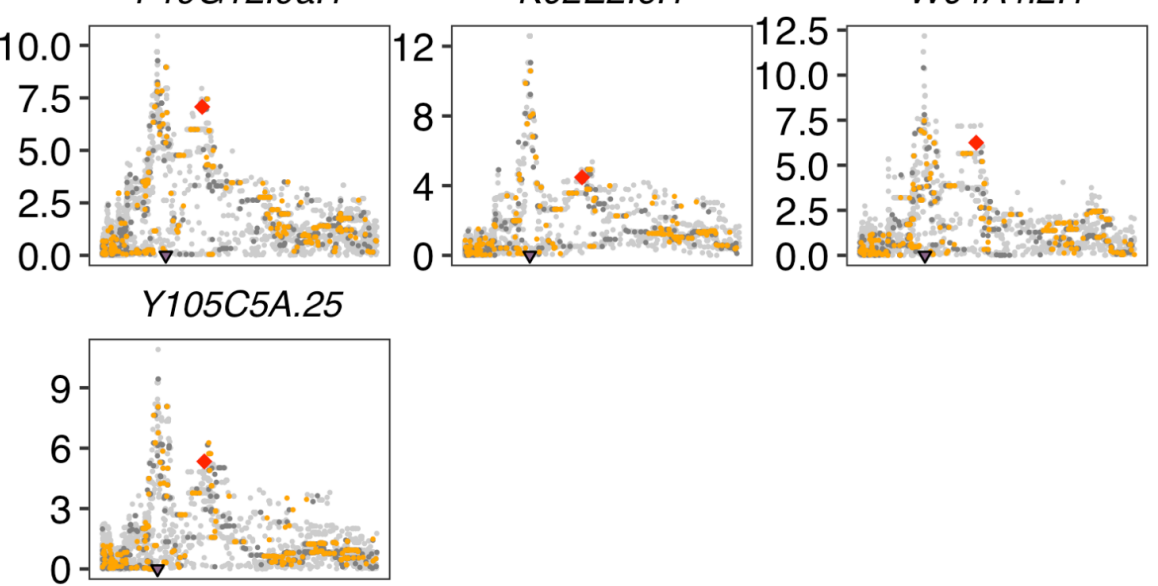

Genomic position (Mb)

Candidate genes $\quad \lim -7$

78

\section{Chromosome I: $39.5 \mathrm{cM}$}

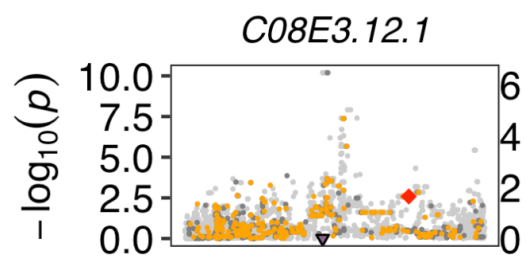

R10E8.6.1

ZK112.2d.1

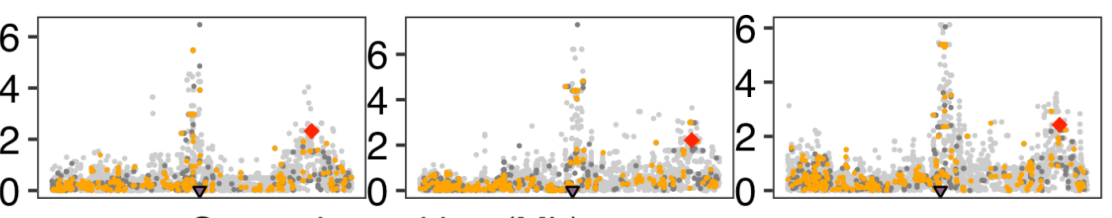

Genomic position (Mb)

Candidate genes $\quad n h r-276$

Chromosome II: $2.5 \mathrm{cM}$

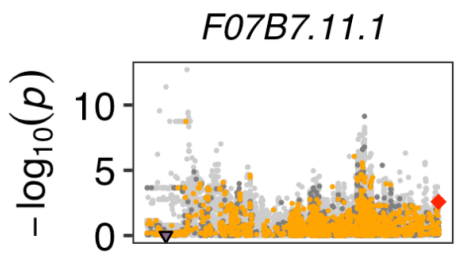

F07B7.4.1

K06C4.12.1

K06C4.4.1
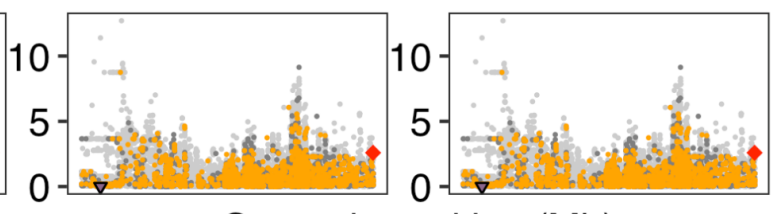

Genomic position (Mb)

Candidate genes $\quad$ T10D4.6 


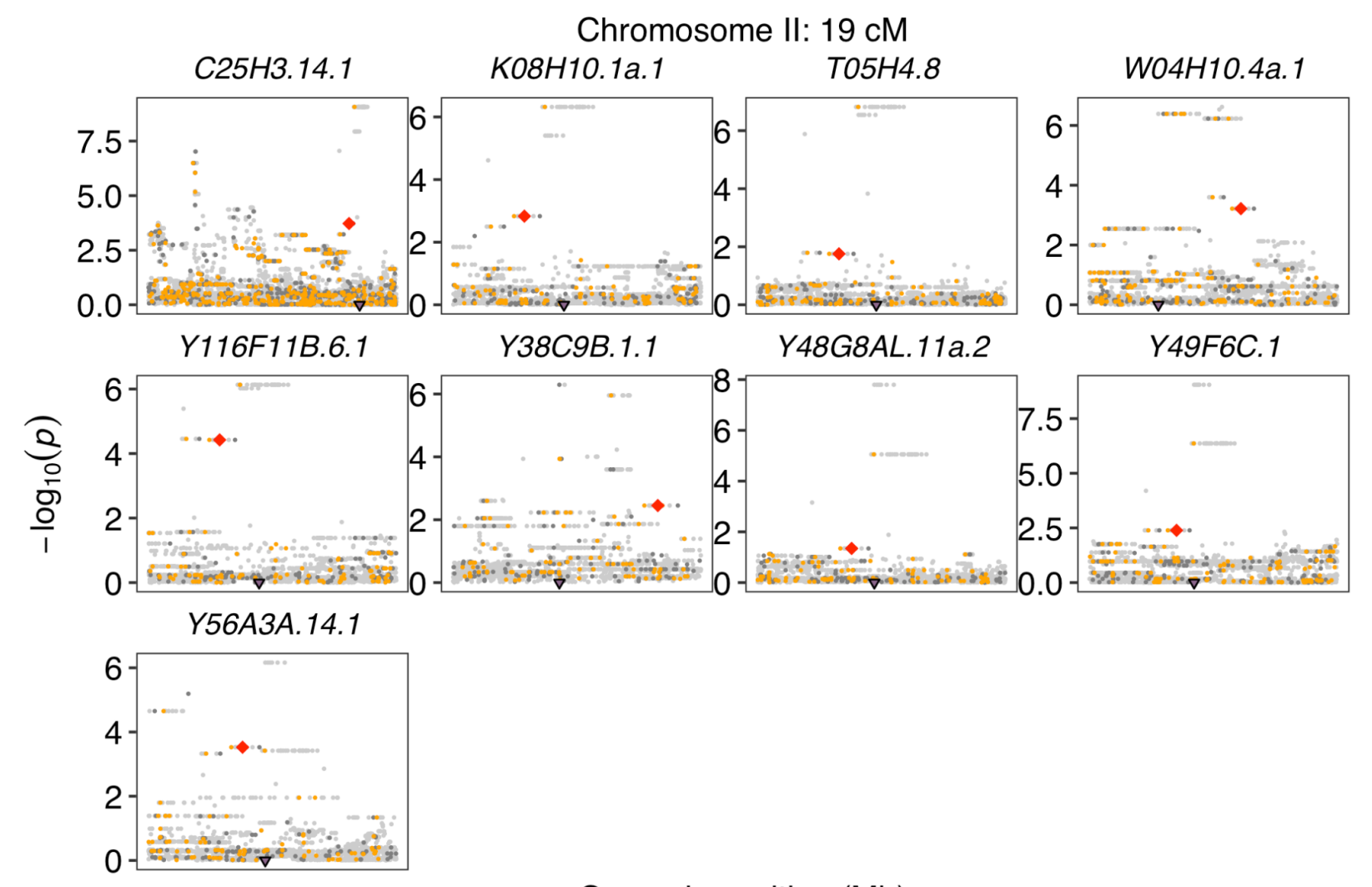

Genomic position (Mb)

80

Candidate genes $\quad$ aptf-4

81

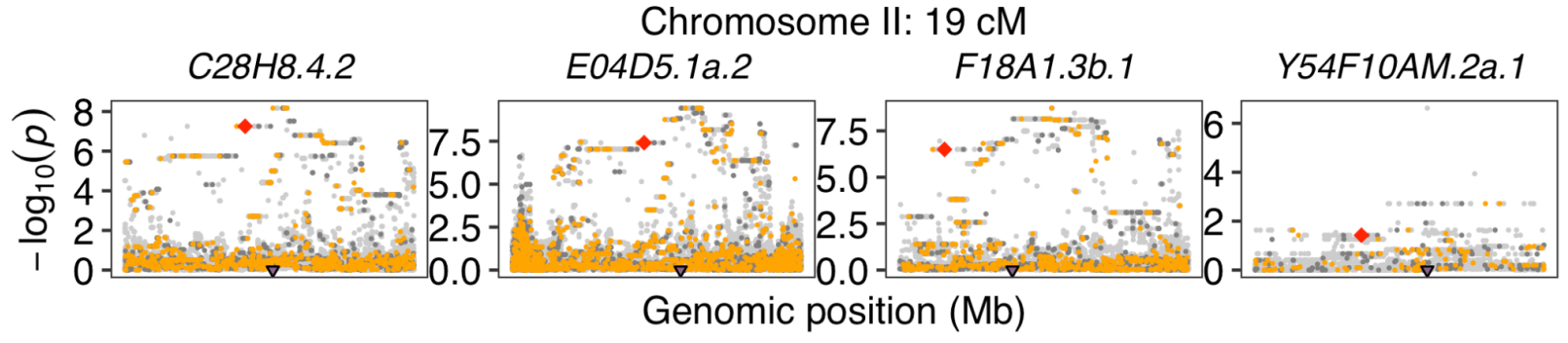

82

\section{Candidate genes $\quad n f i-1$}

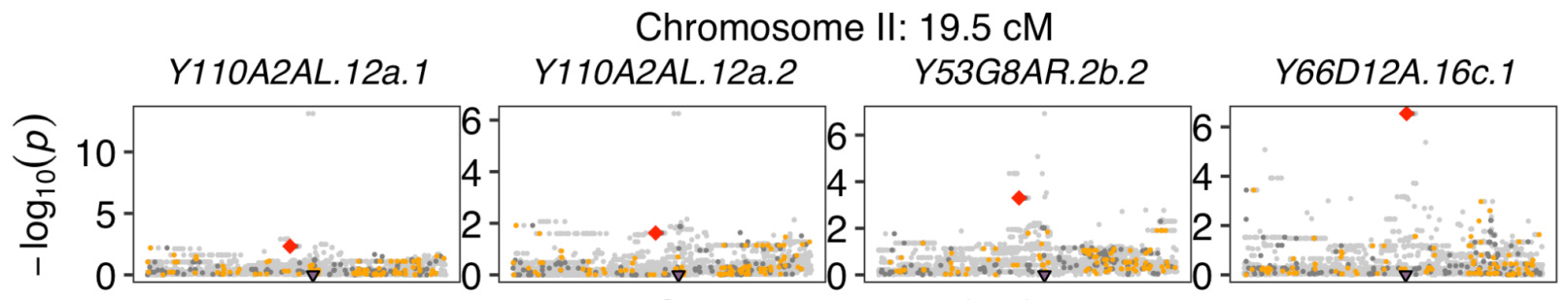

Genomic position (Mb)

Candidate genes $\quad$ jun-1 
83

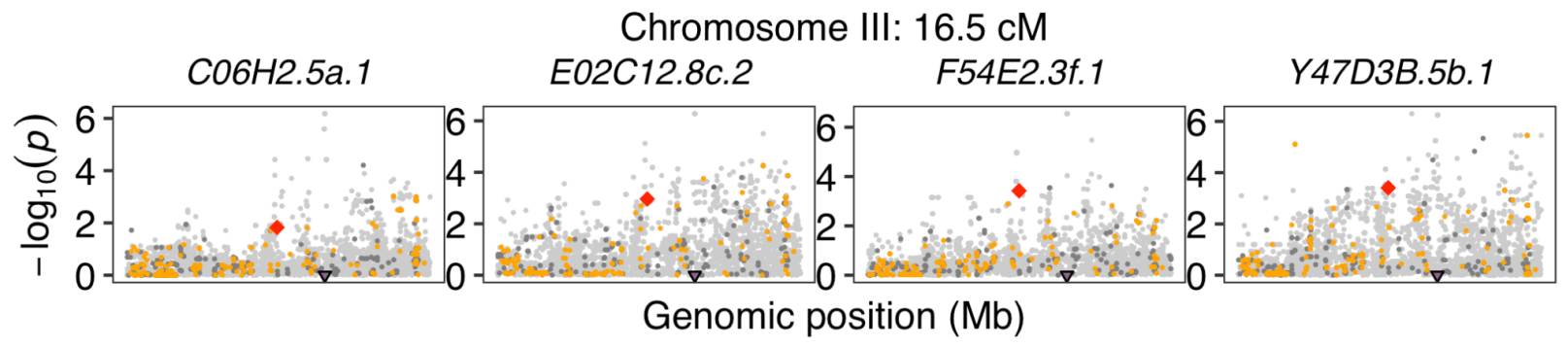

84

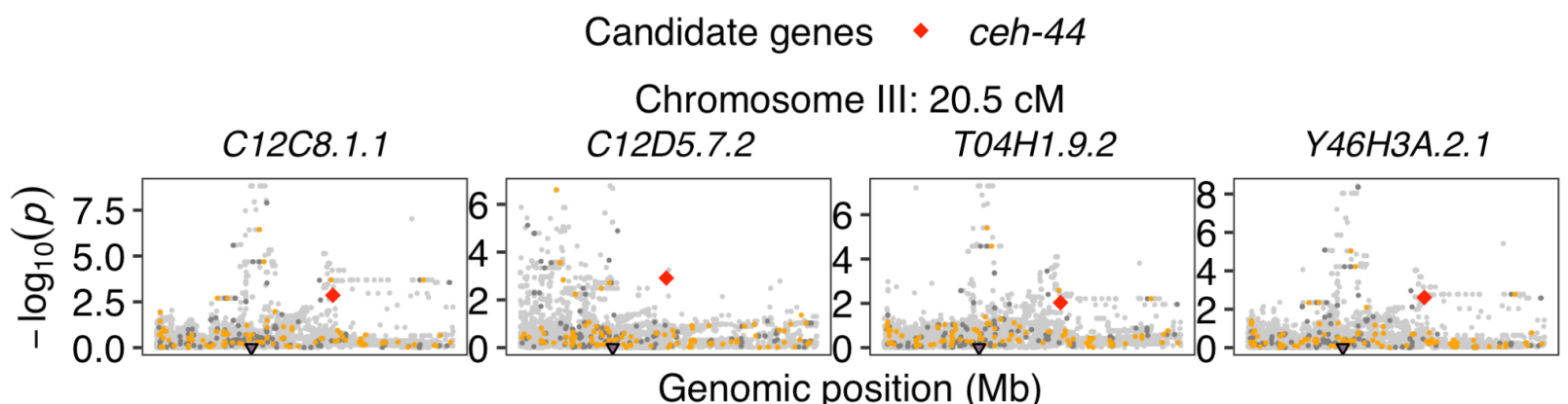

Genomic position (Mb)

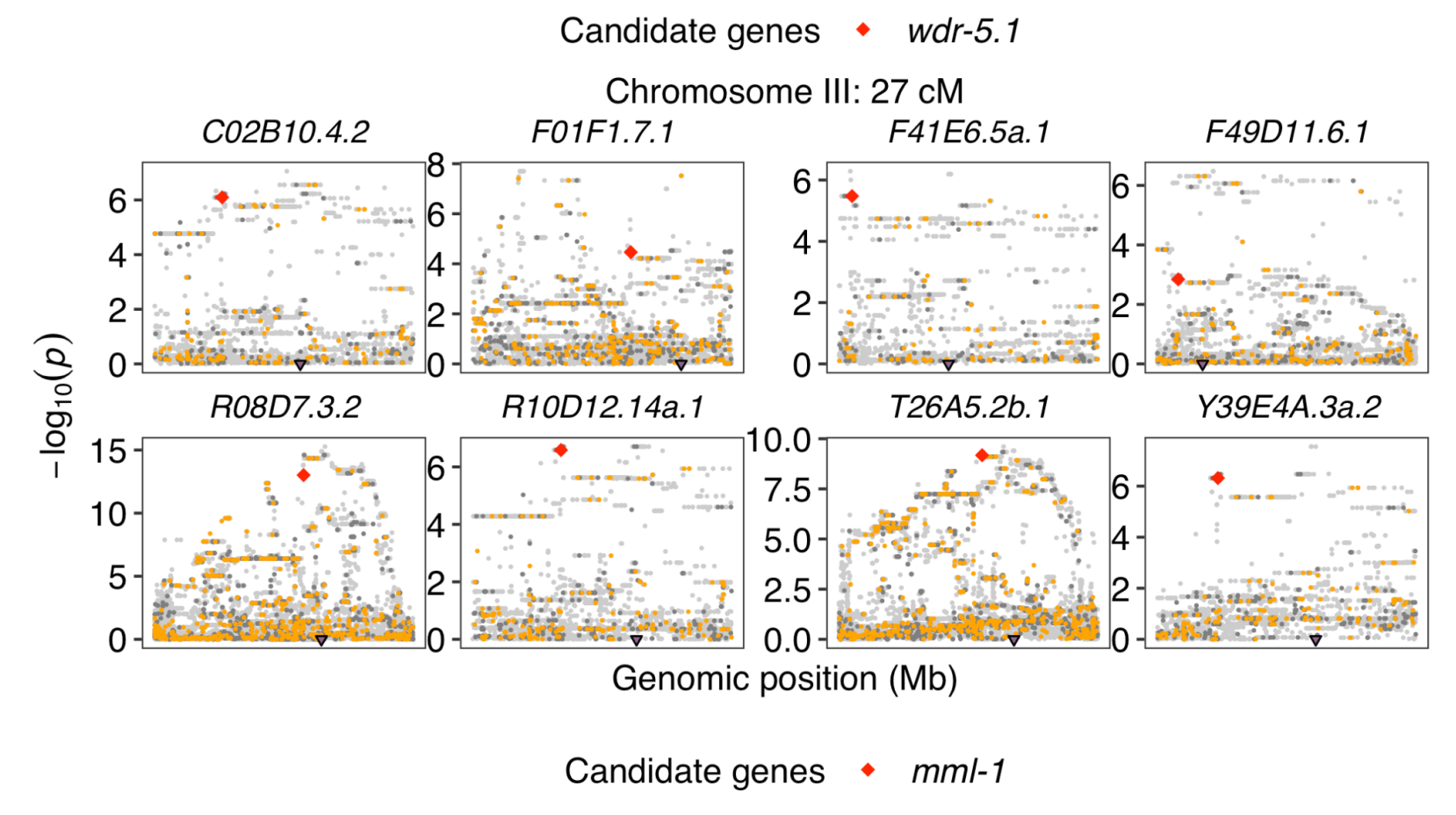




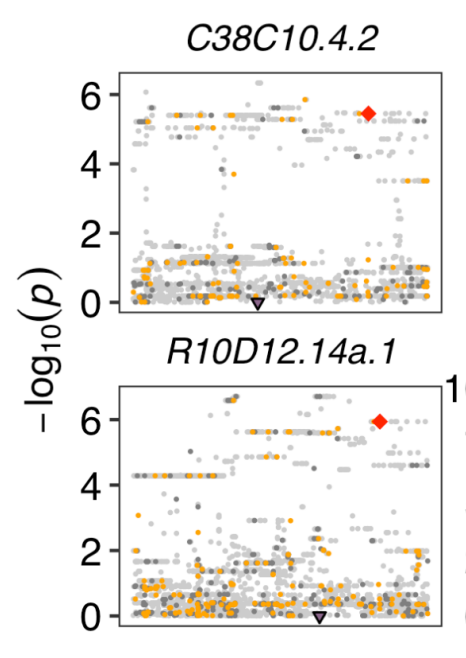

86

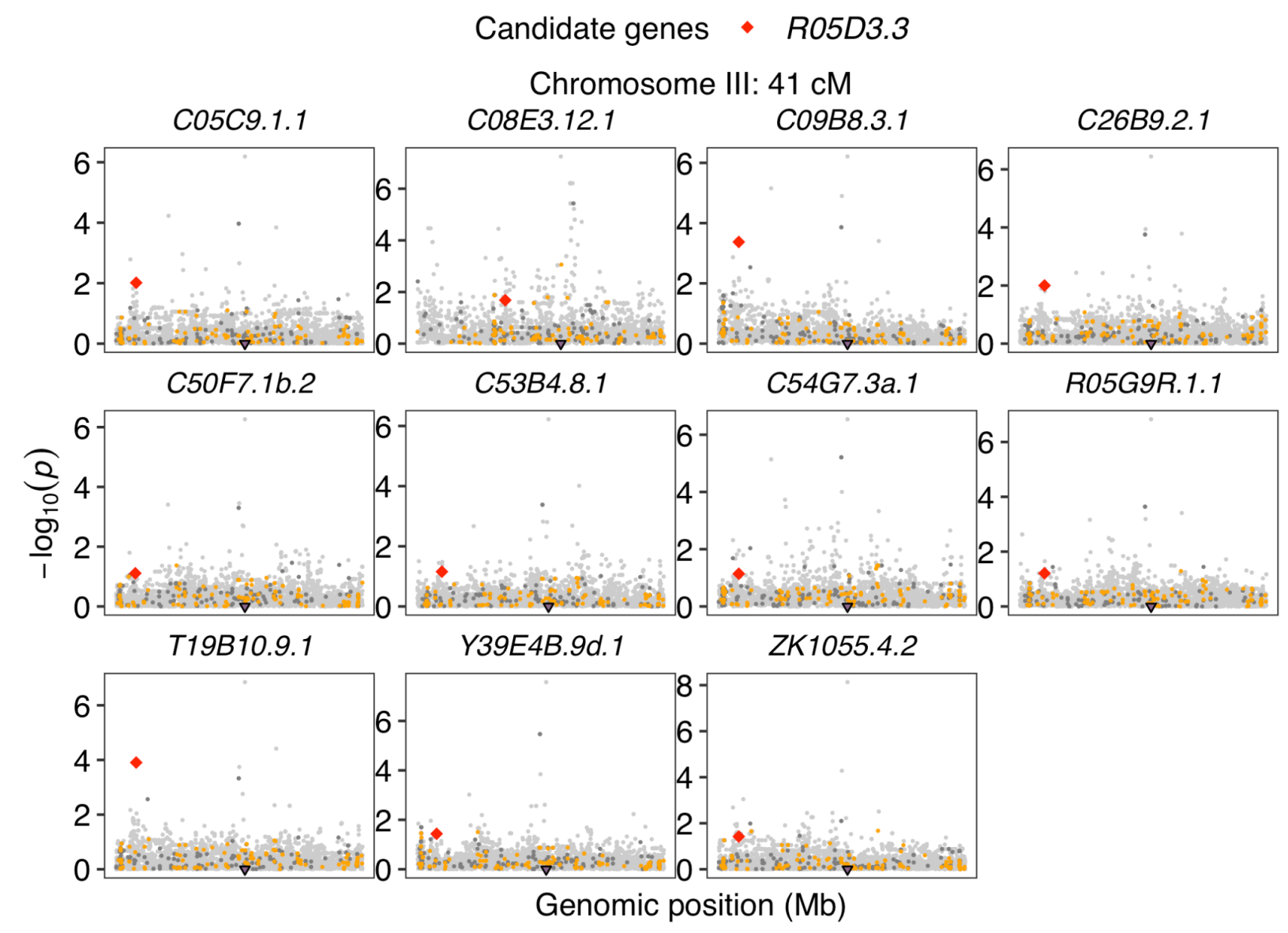

87

Chromosome III: $27 \mathrm{cM}$
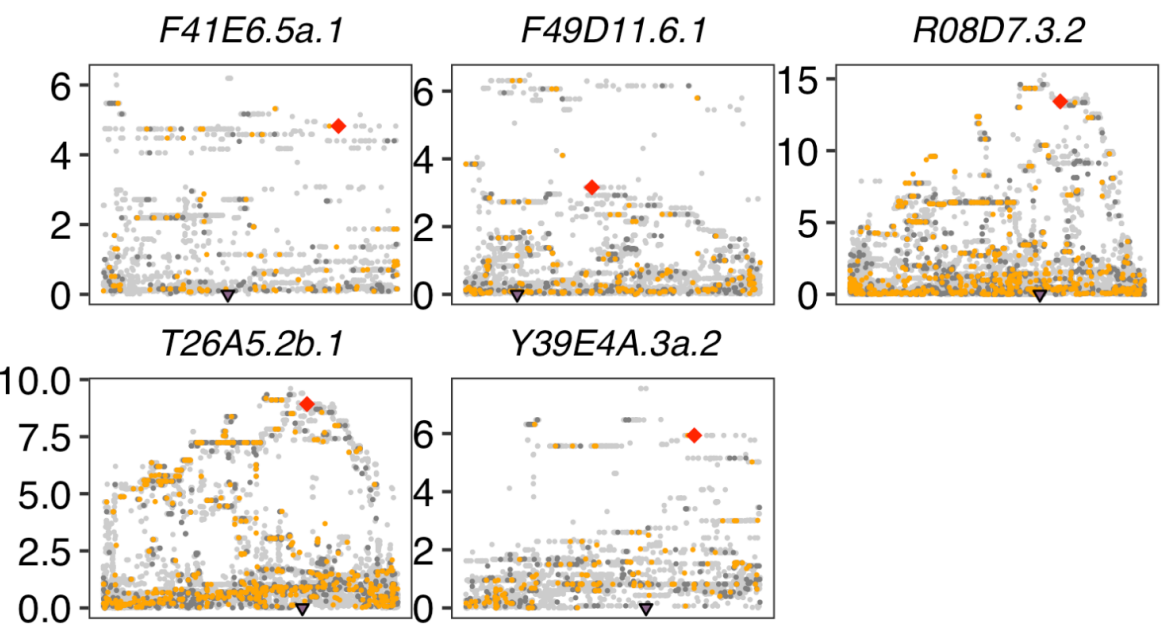

Genomic position (Mb)

Chromosome III: $41 \mathrm{cM}$ 


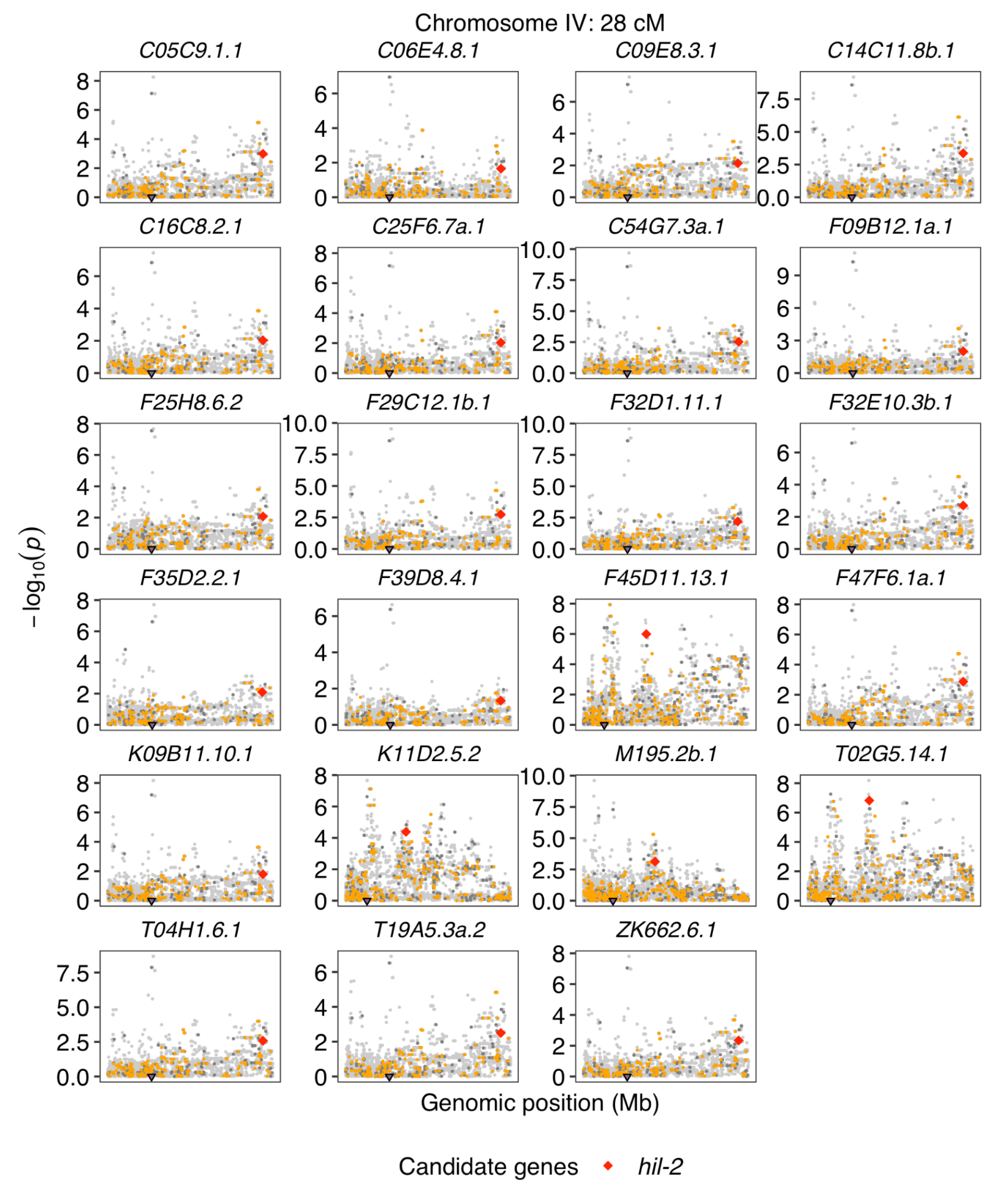




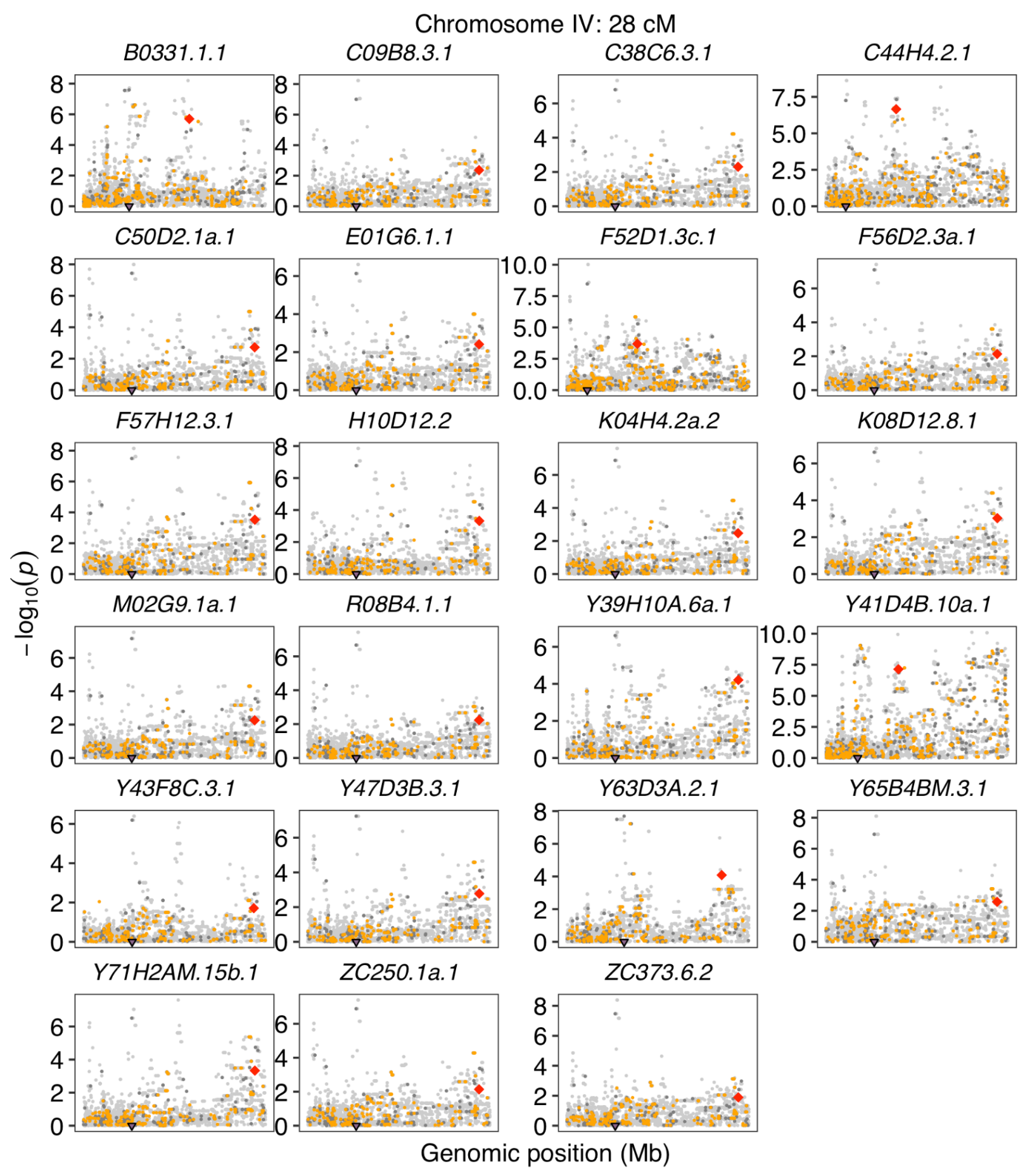


90
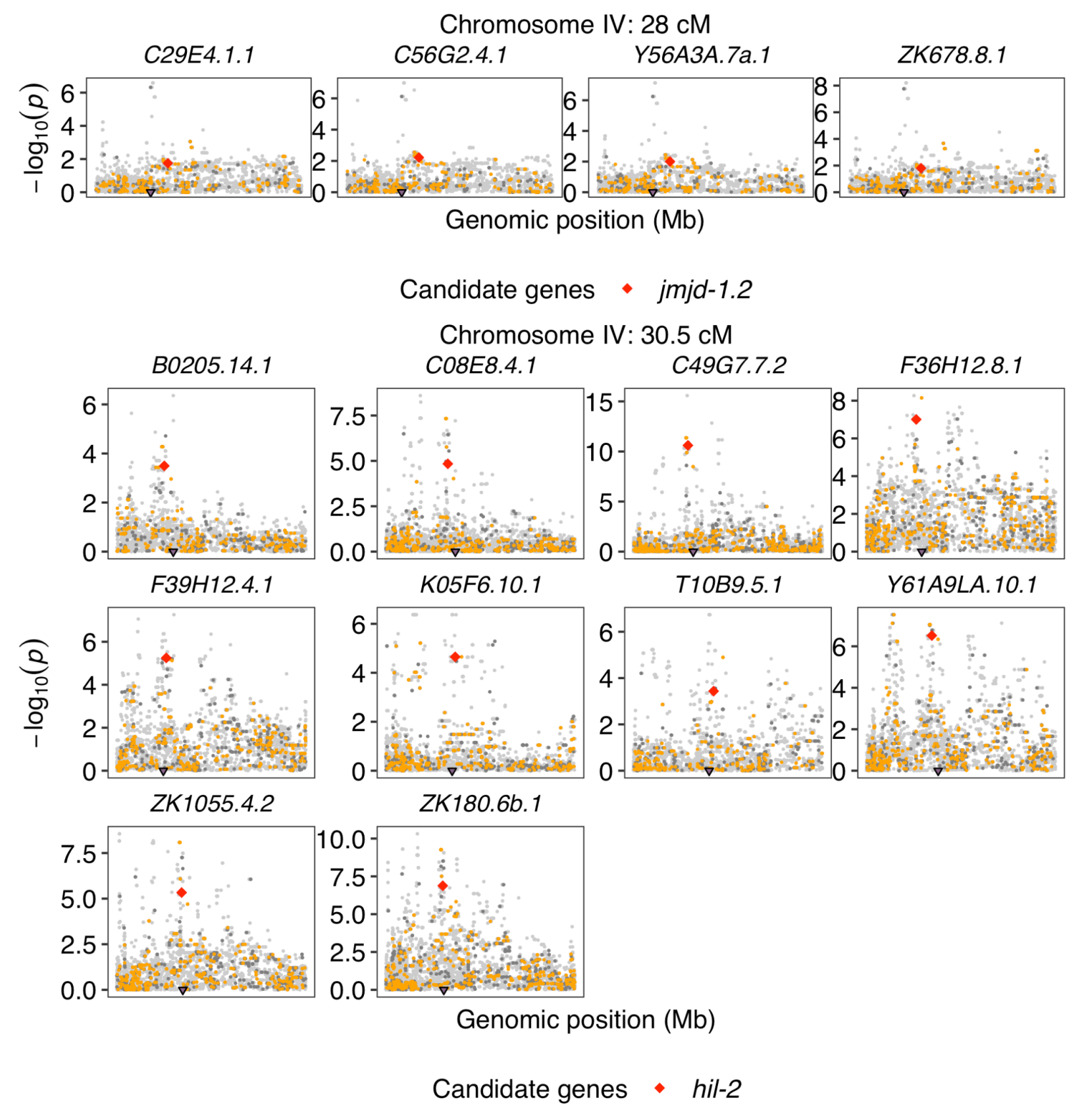


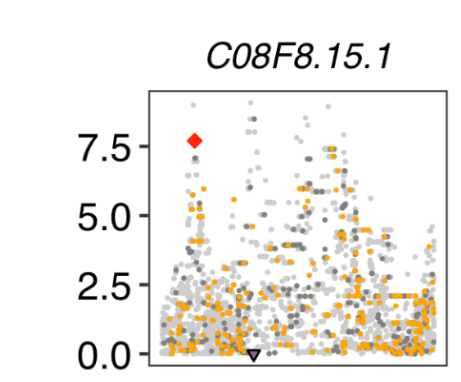

F48E3.4.1
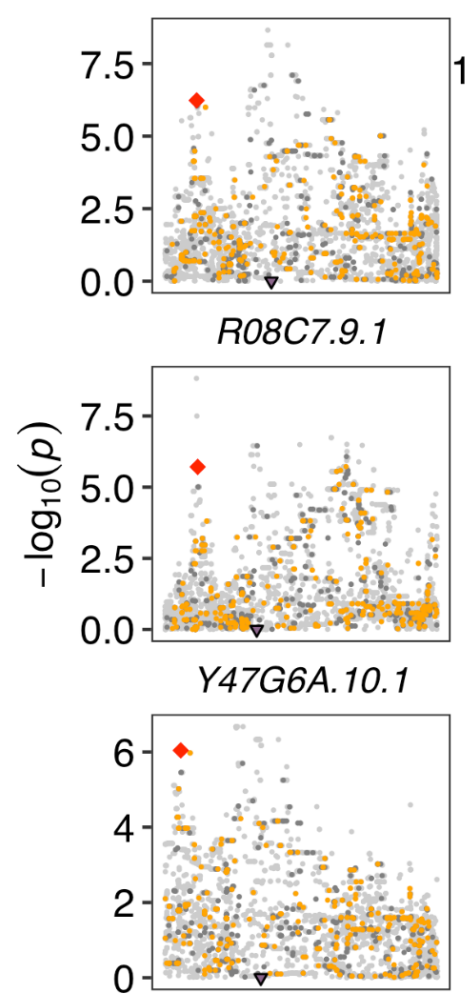

ZK993.1a.1

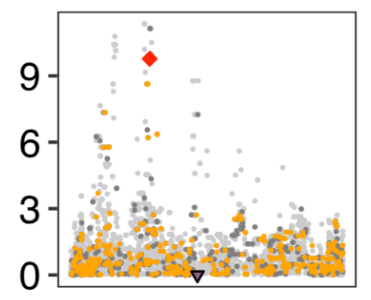

Chromosome IV: $31 \mathrm{cM}$

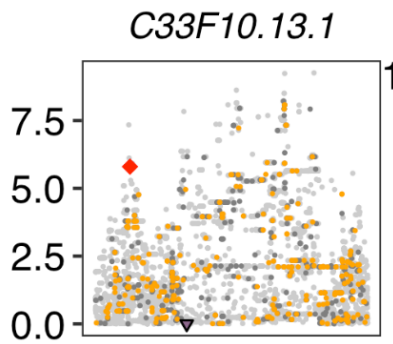

F56A12.1.1
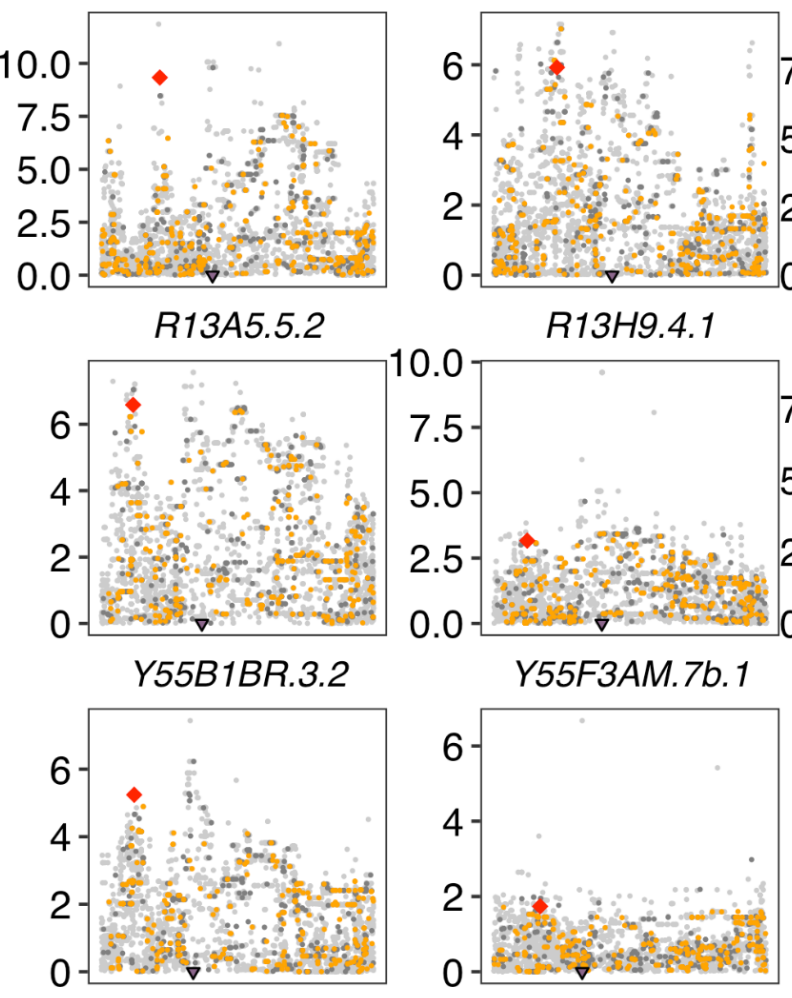

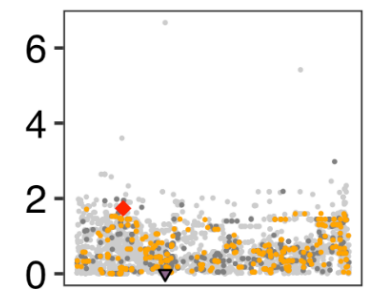

F46F3.1a.1

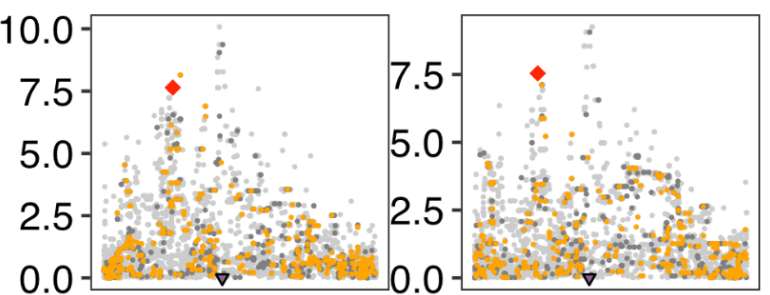

R08C7.13.1

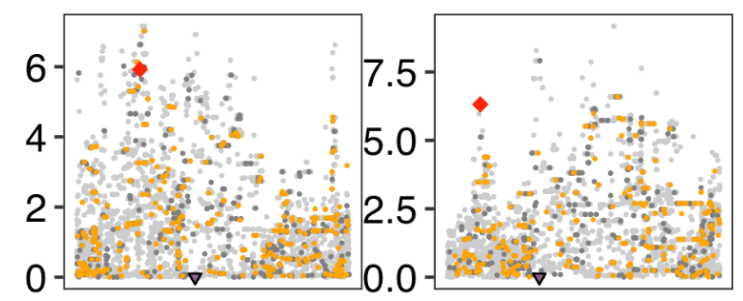

T14G12.4a.1

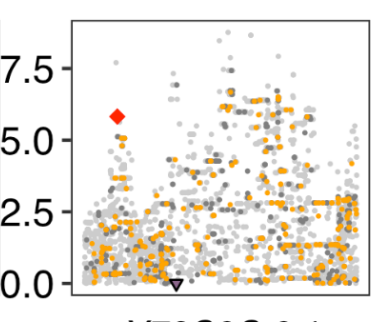

Y73C8C.8.1

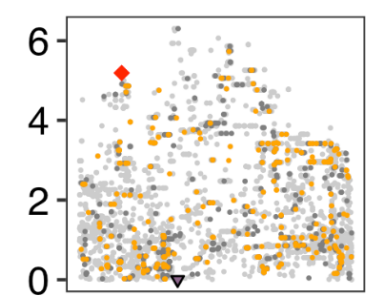

Genomic position (Mb)

Candidate genes $\quad$ hil-2 


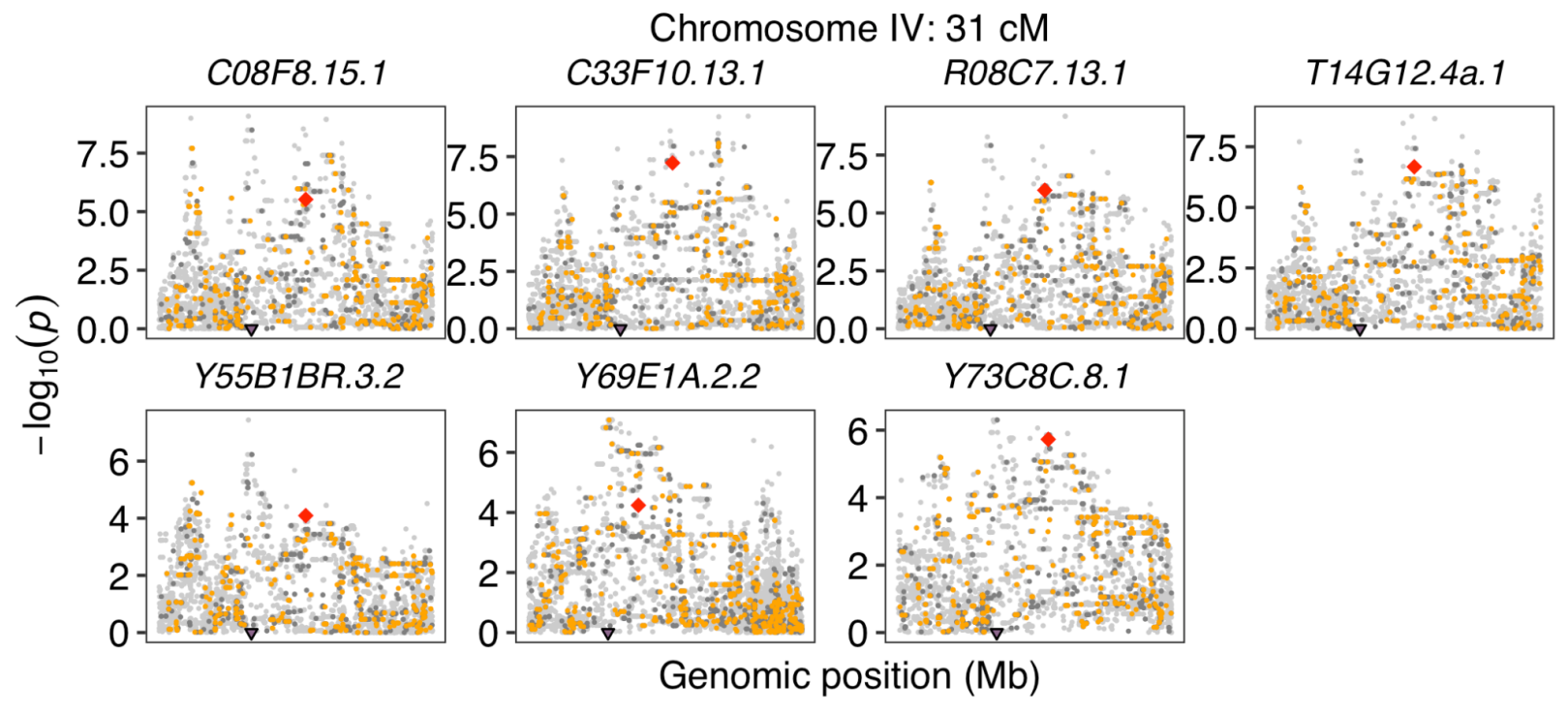

94
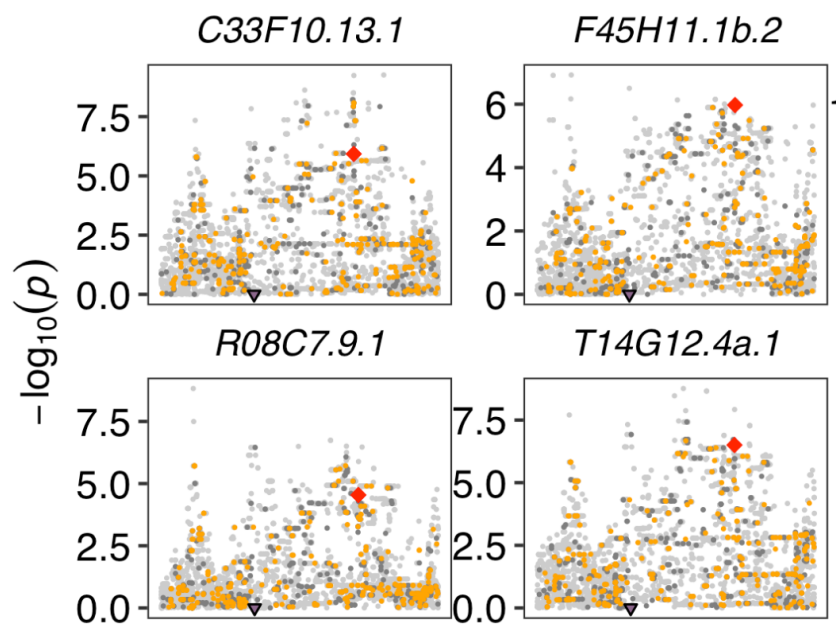

Chromosome IV: $31 \mathrm{cM}$

Candidate genes $\quad n h r-264$

T14G12.4a.1
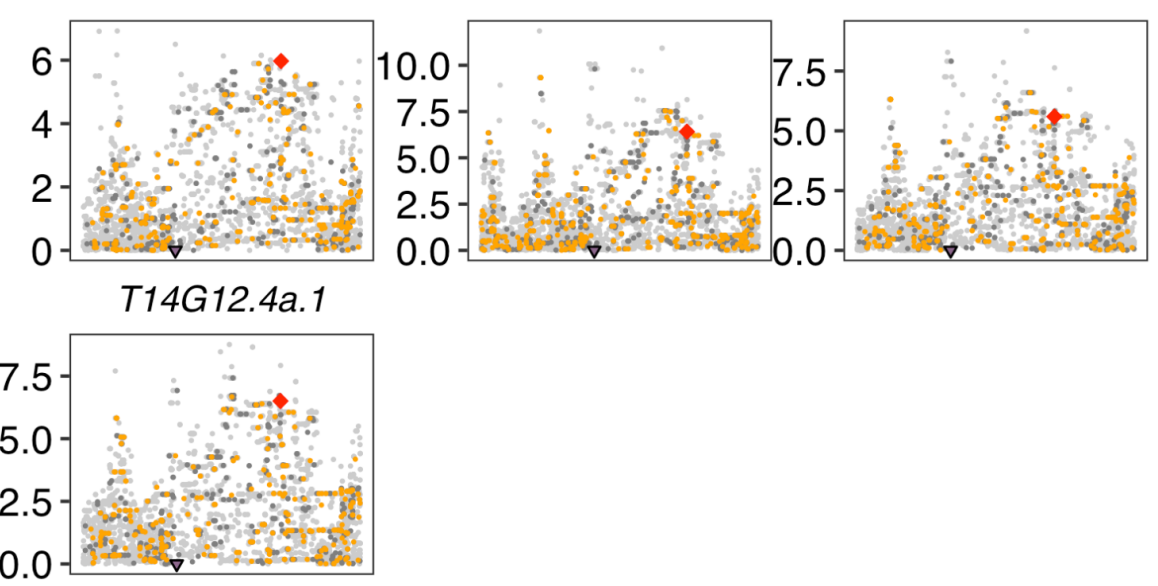

Genomic position (Mb)

Candidate genes $\quad$ zim-2

F44E5.4.1

Chromosome IV: $31.5 \mathrm{cM}$

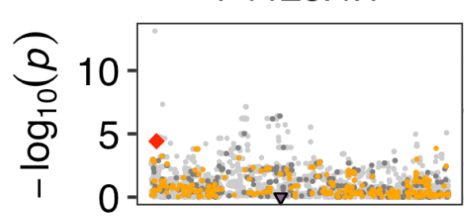

F48E3.6.1

T27E4.2.1

W05F2.2
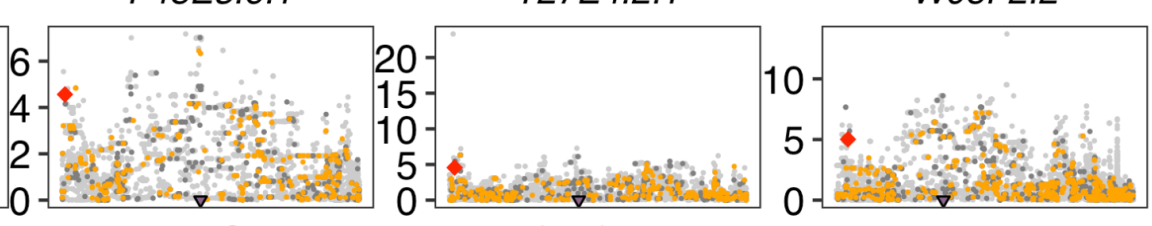

Genomic position (Mb) 
96
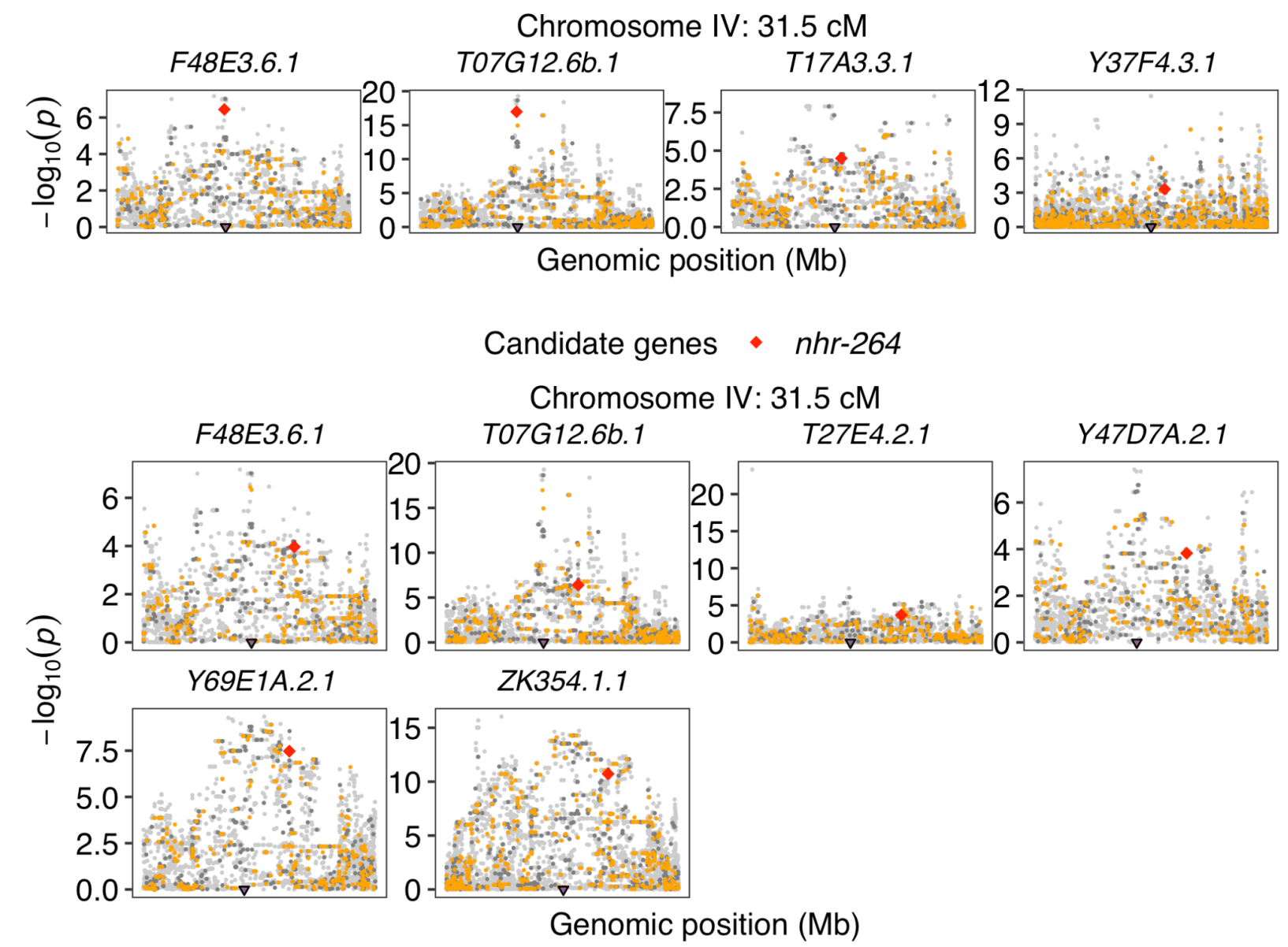

97

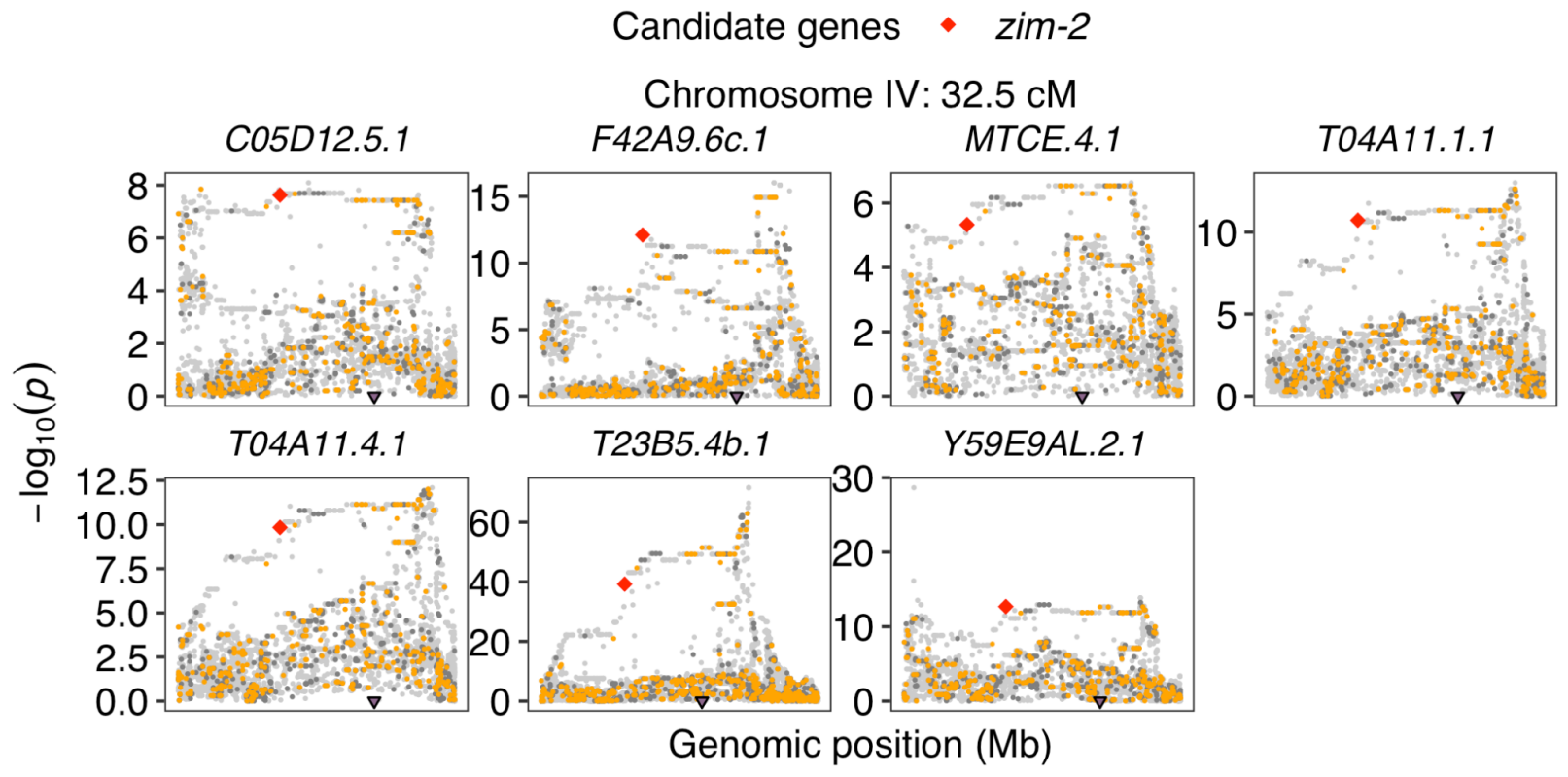


99

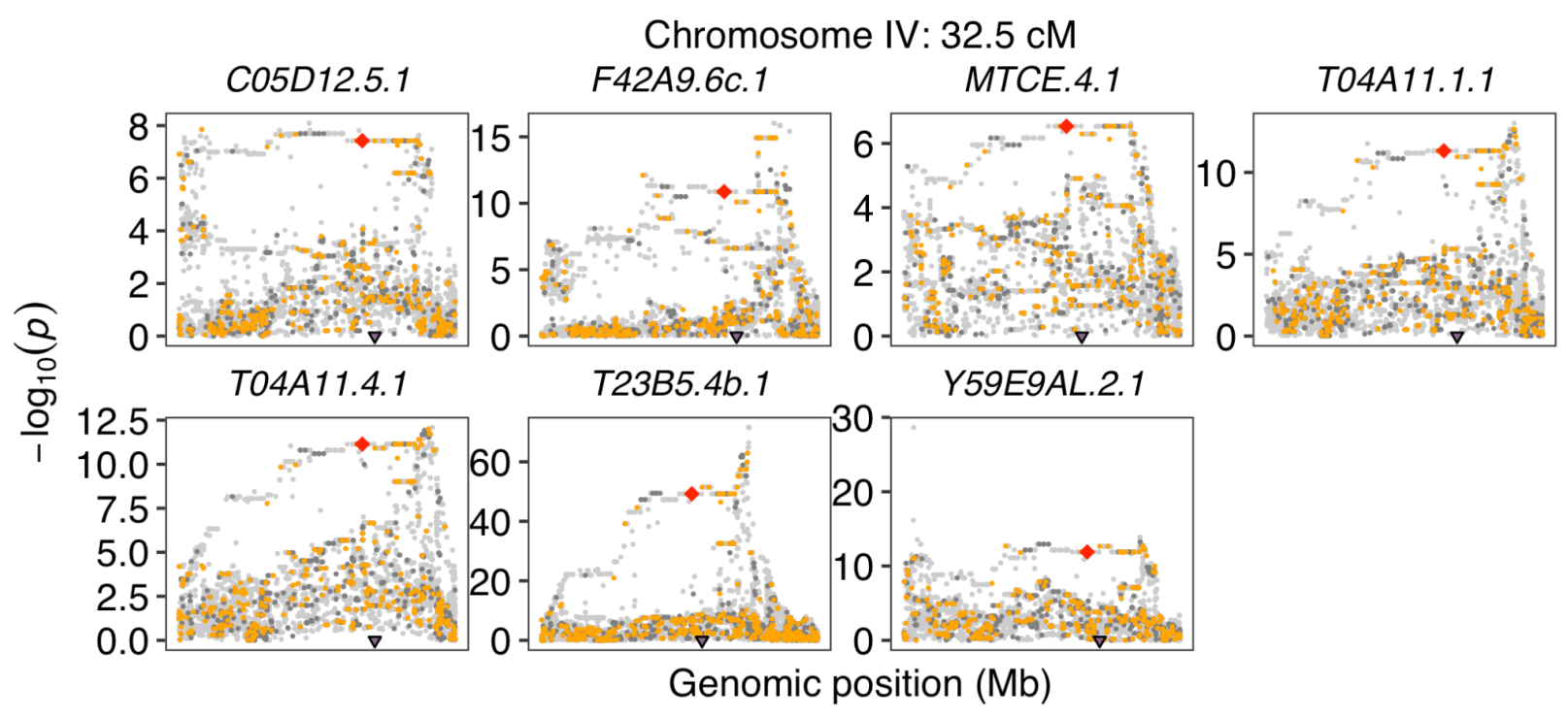

100

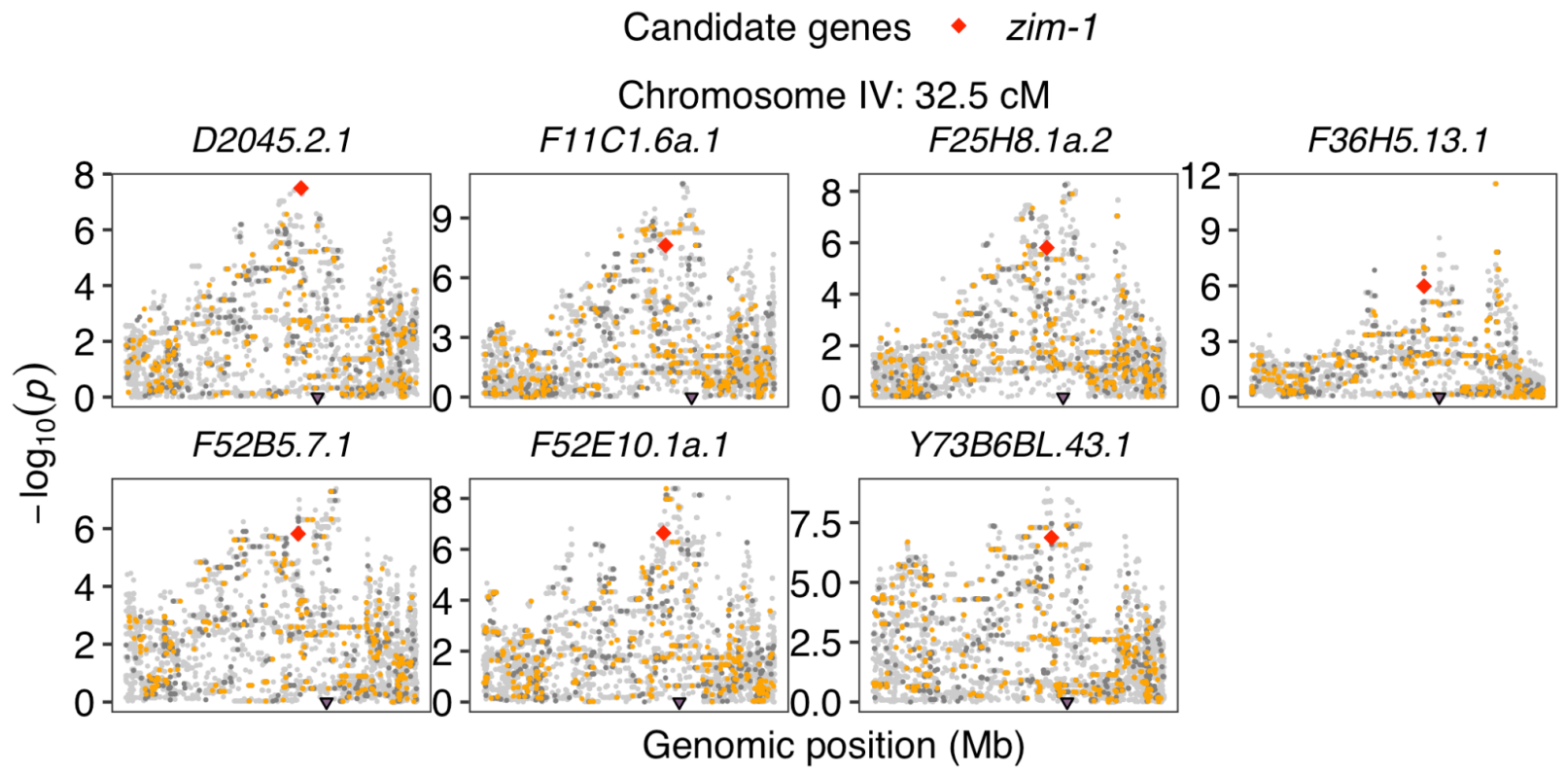

Candidate genes $\quad$ zim-2

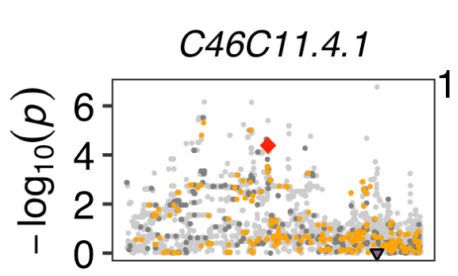

Chromosome IV: $33.5 \mathrm{cM}$

F45D11.8

T25B9.8.2

Y37H2A.8
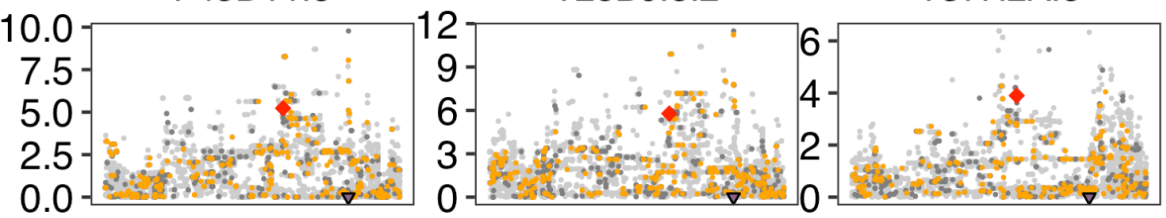

Genomic position (Mb)

Candidate genes $\quad$ zim-3 
102

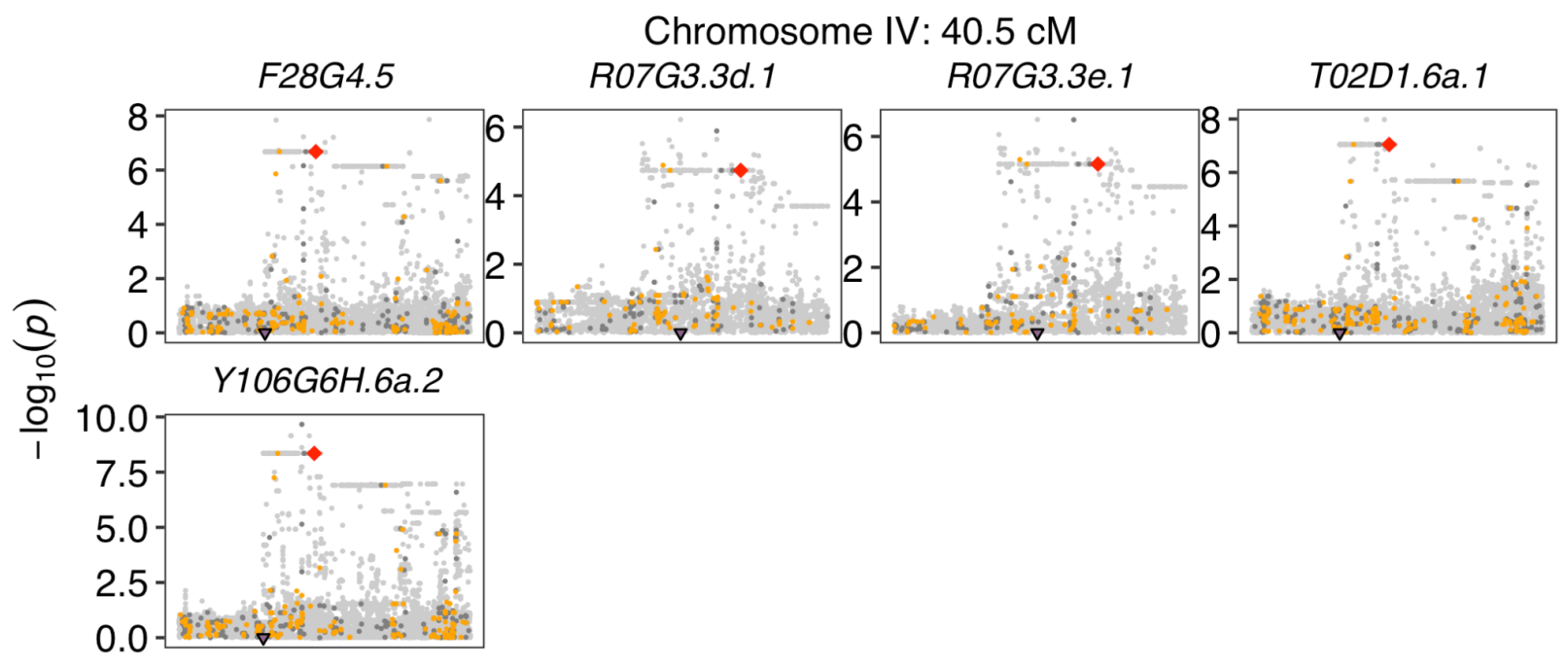

Genomic position (Mb)

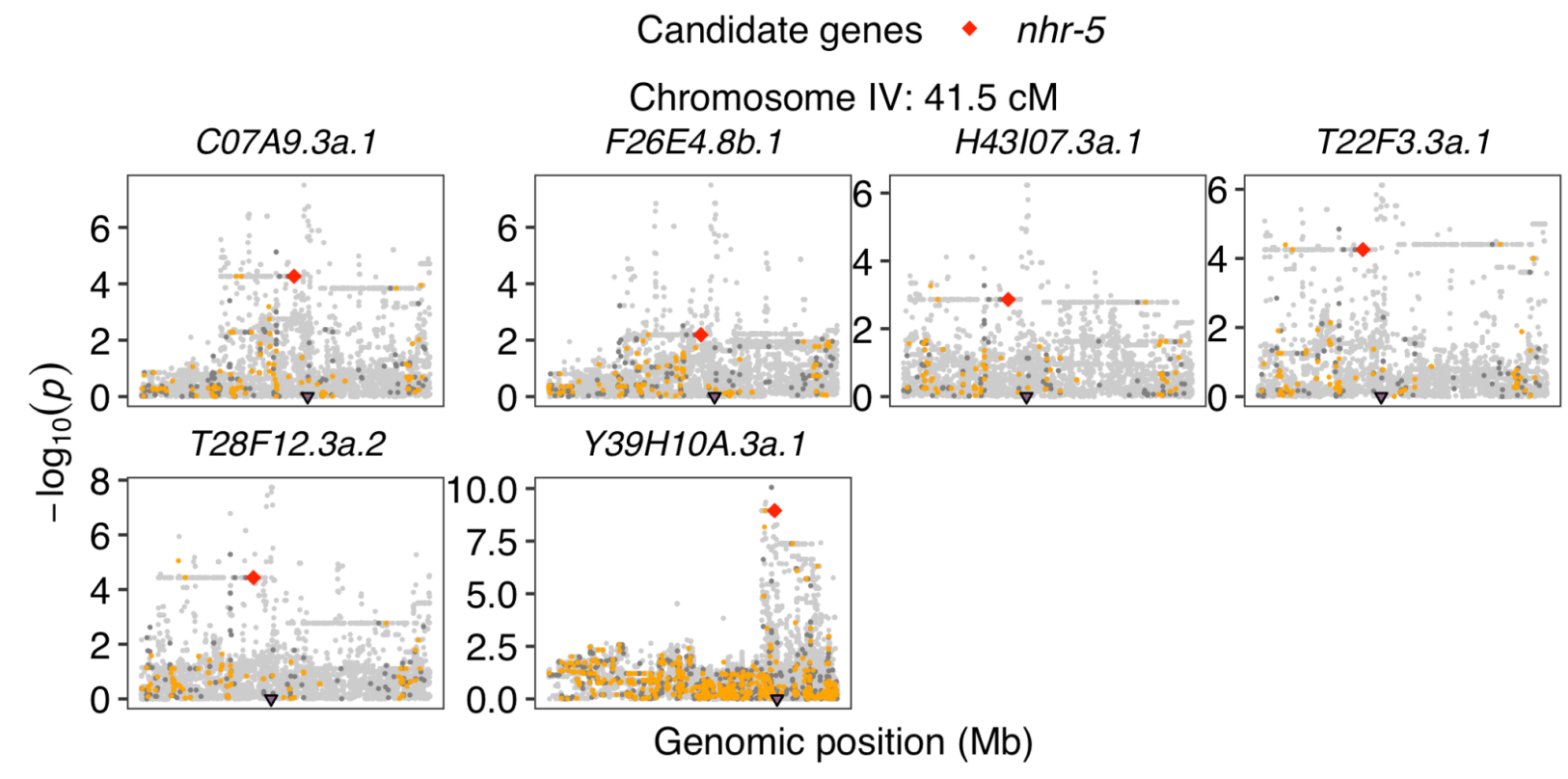

103

Candidate genes * nhr-5

104 


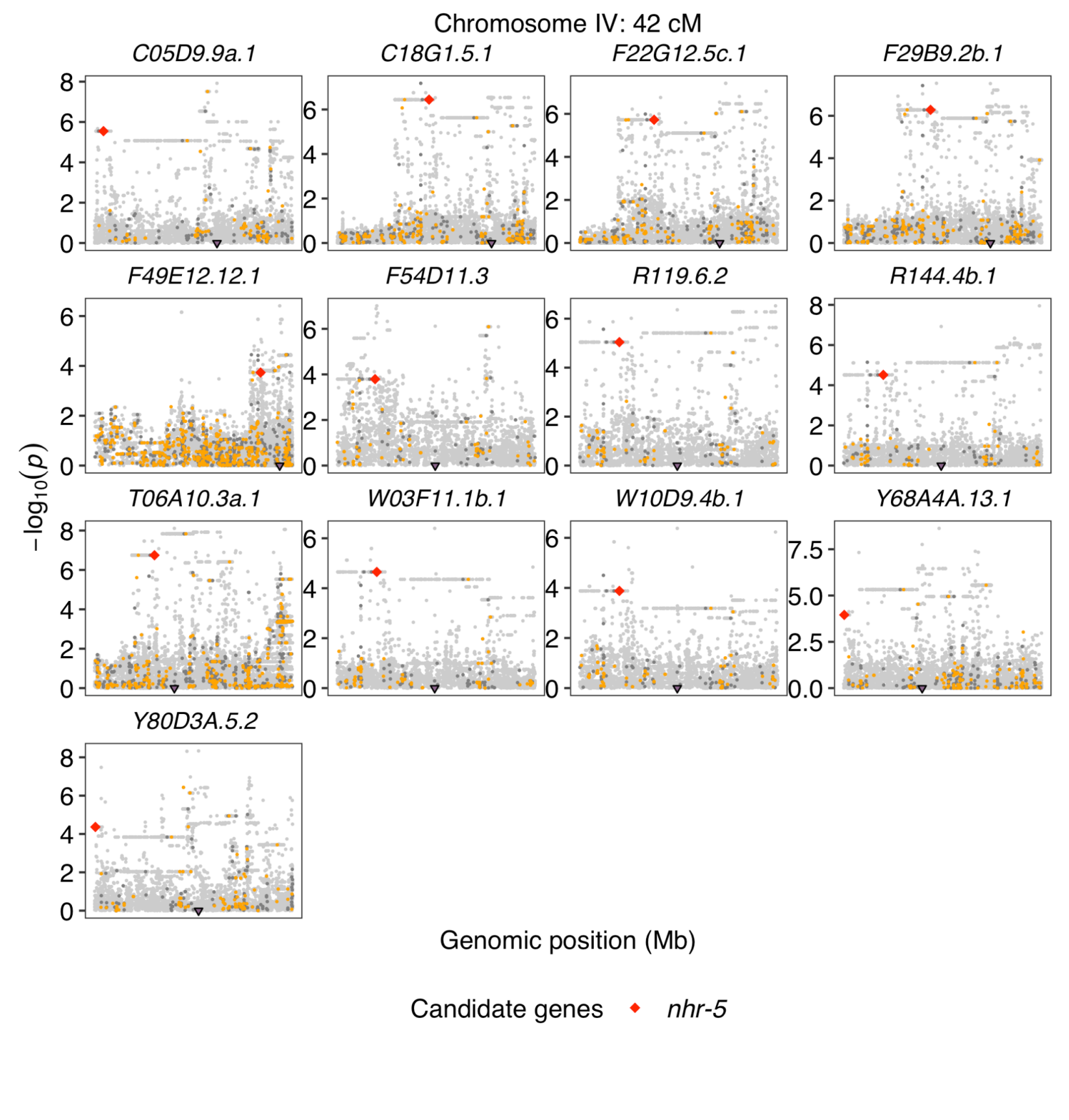


108

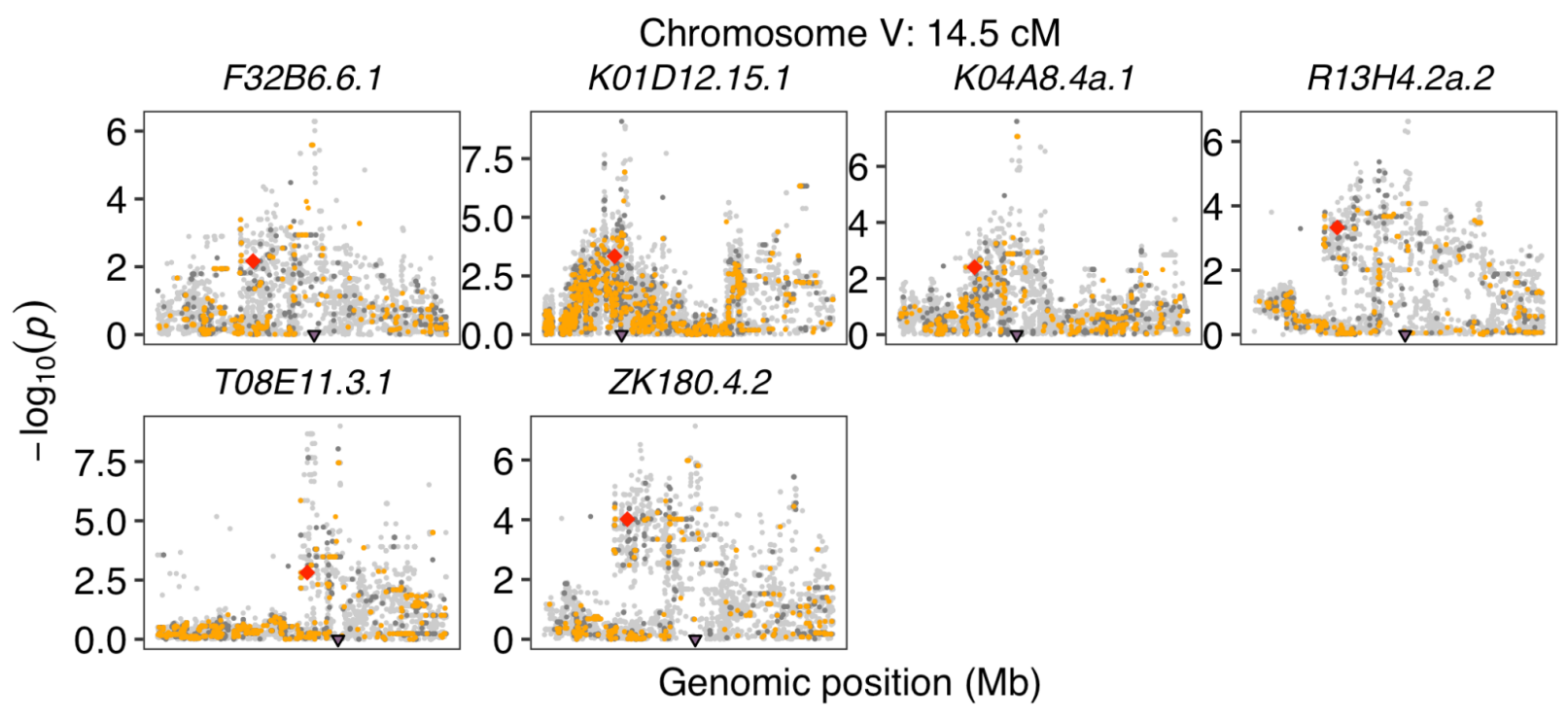

109

Candidate genes $\quad n h r-181$

Chromosome V: $21 \mathrm{cM}$

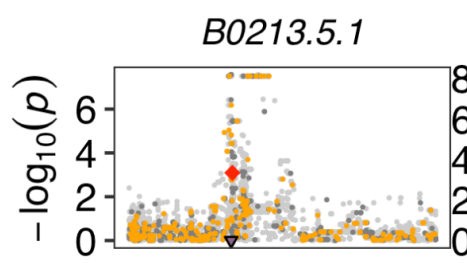

C08G5.4b.1

T13H5.3.1

T23B12.5.1

110

Candidate genes $\quad n h r-148$ 


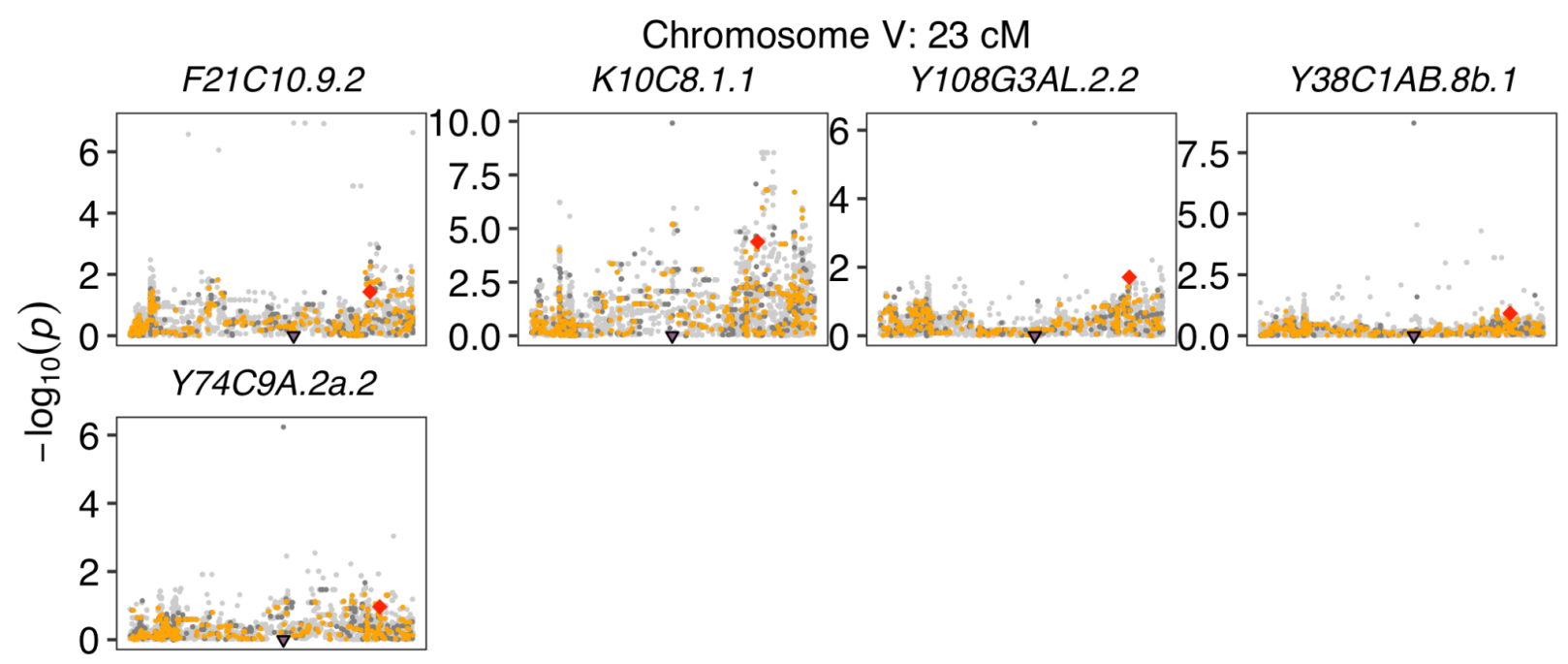

Genomic position (Mb)

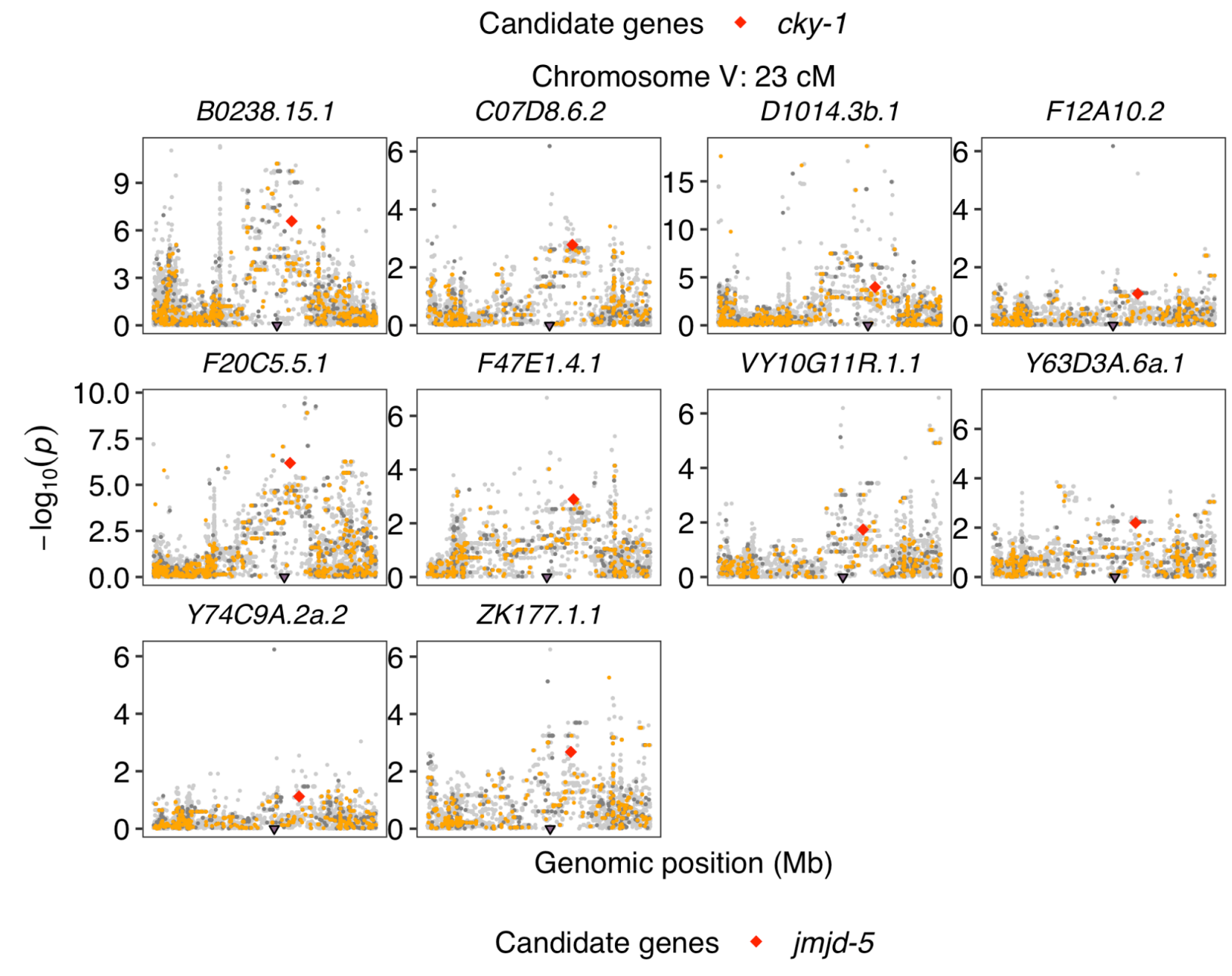




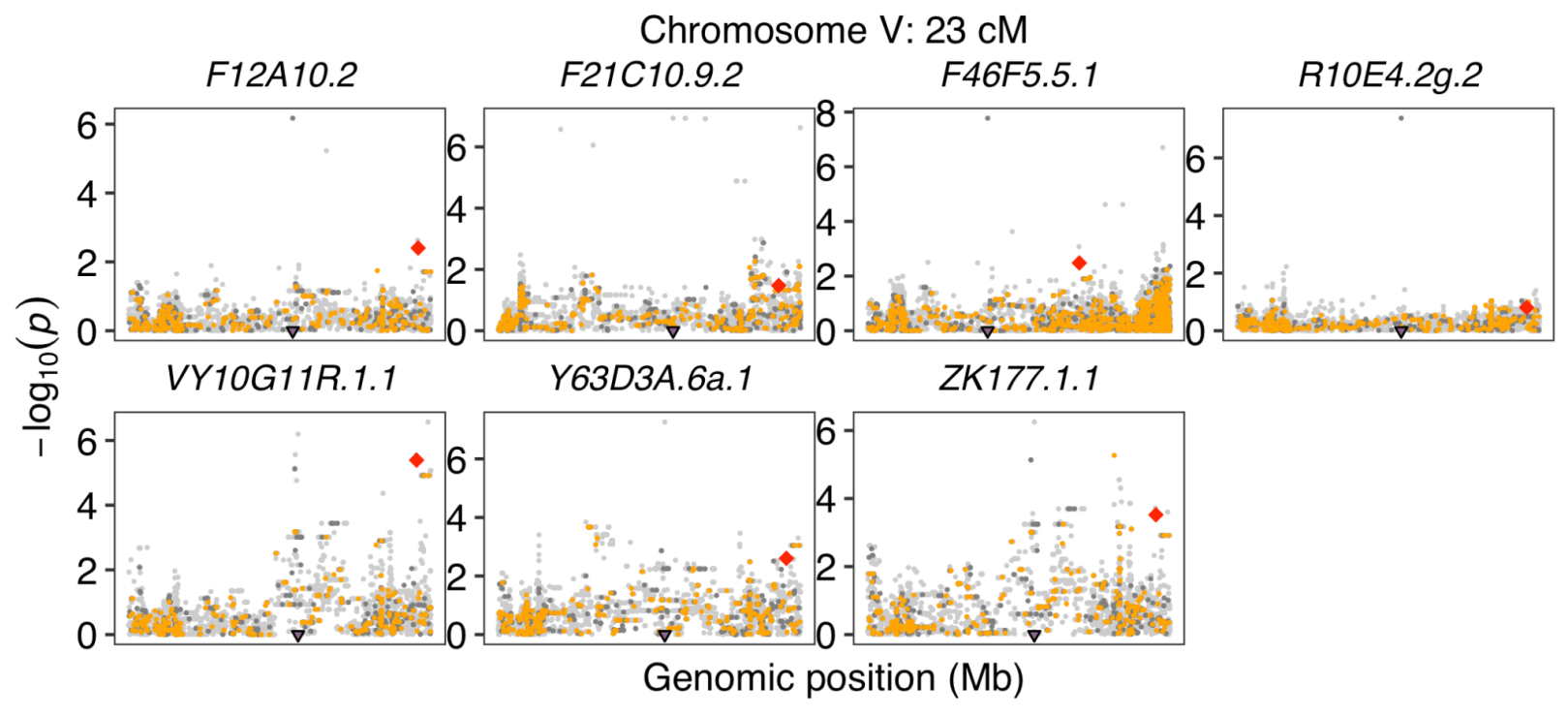

114

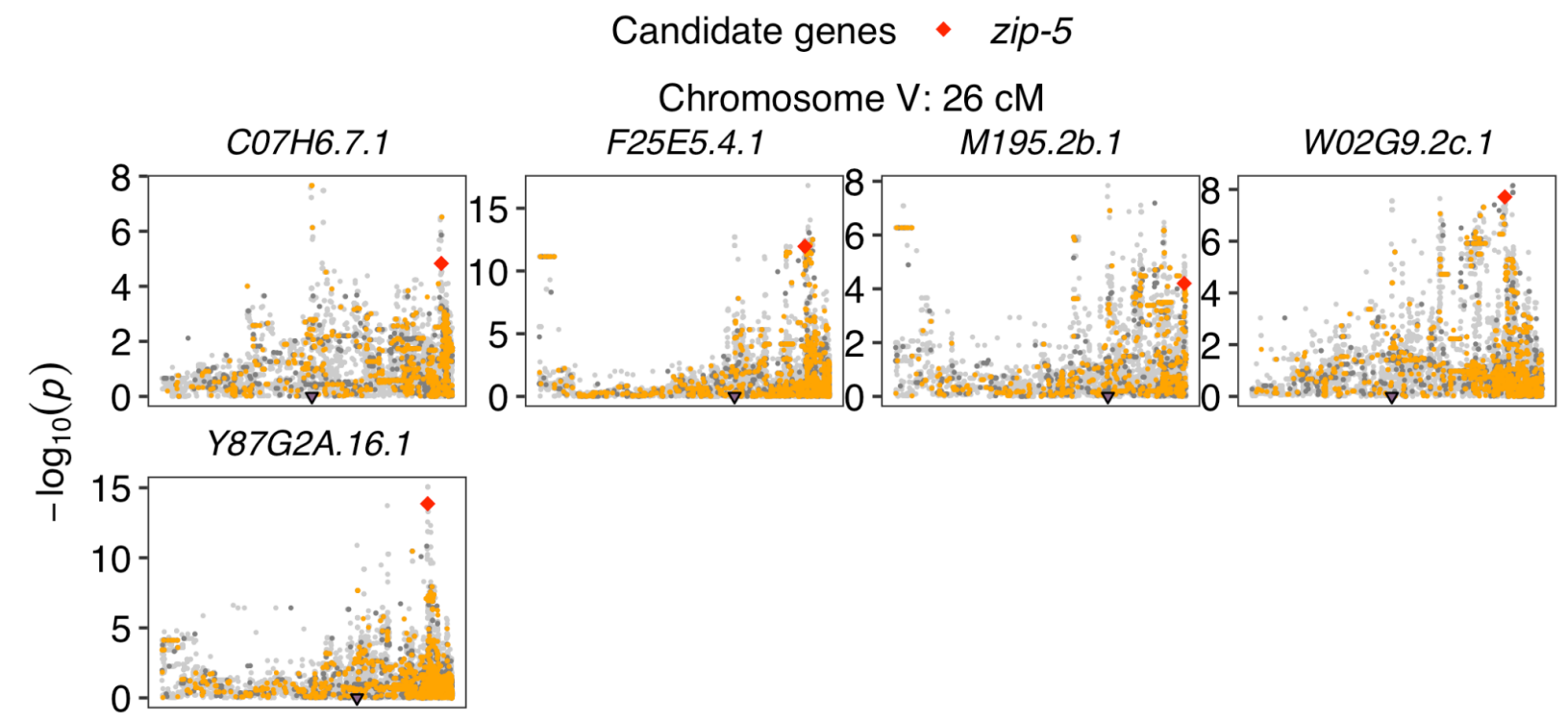

Genomic position (Mb)

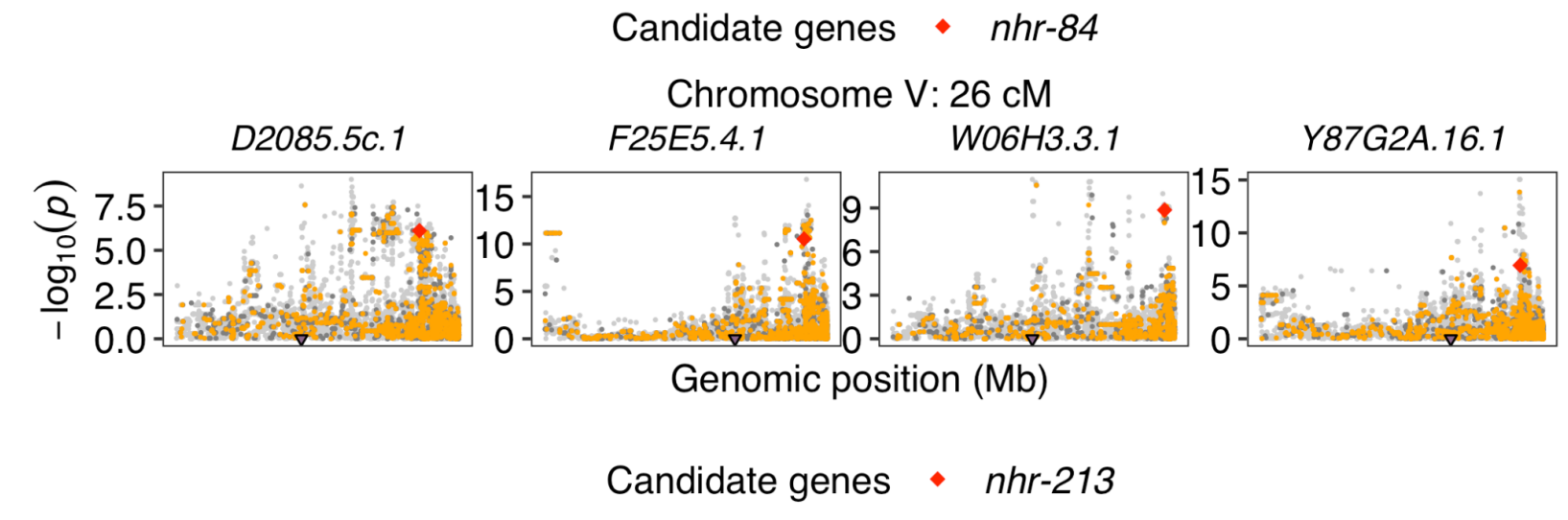



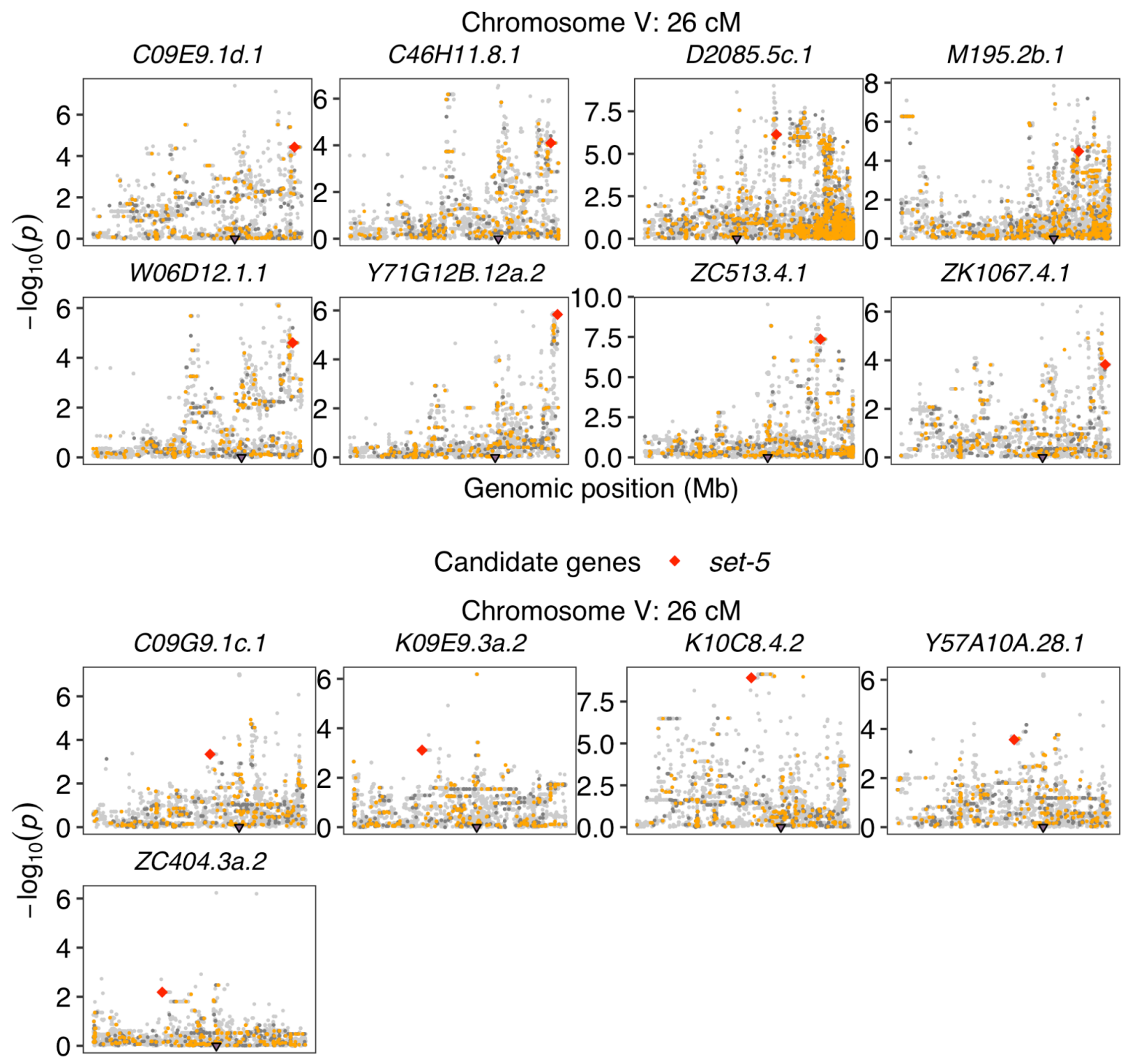

Genomic position (Mb)

117

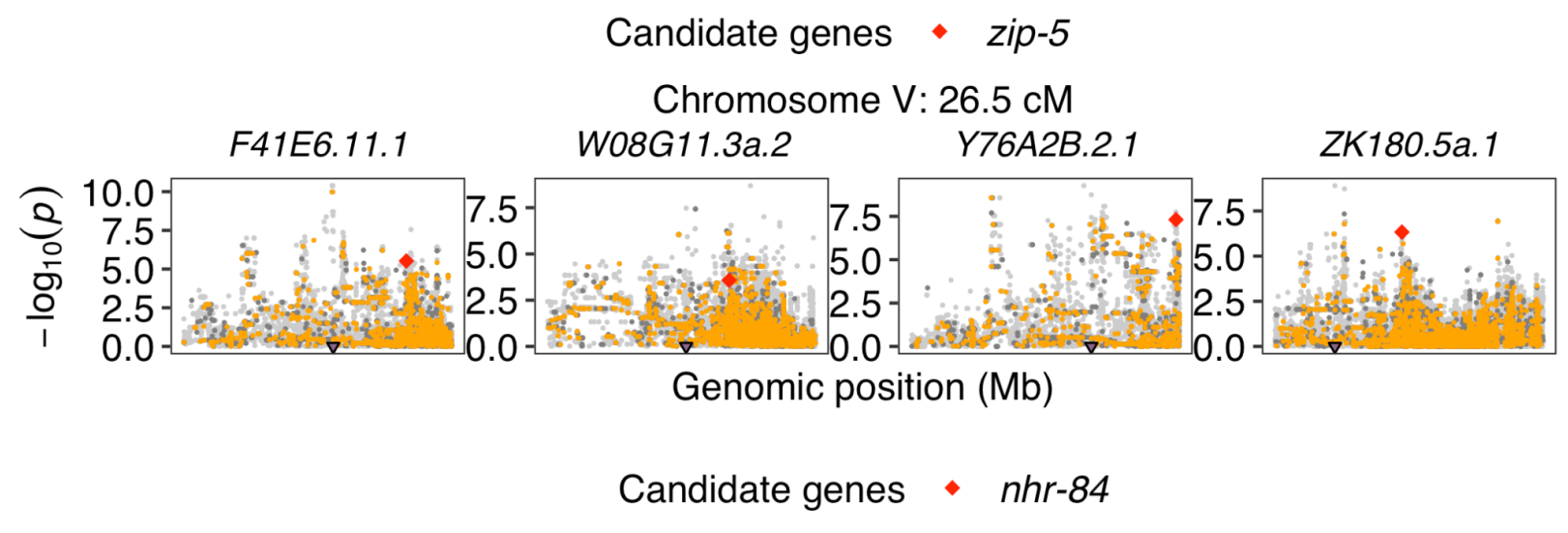




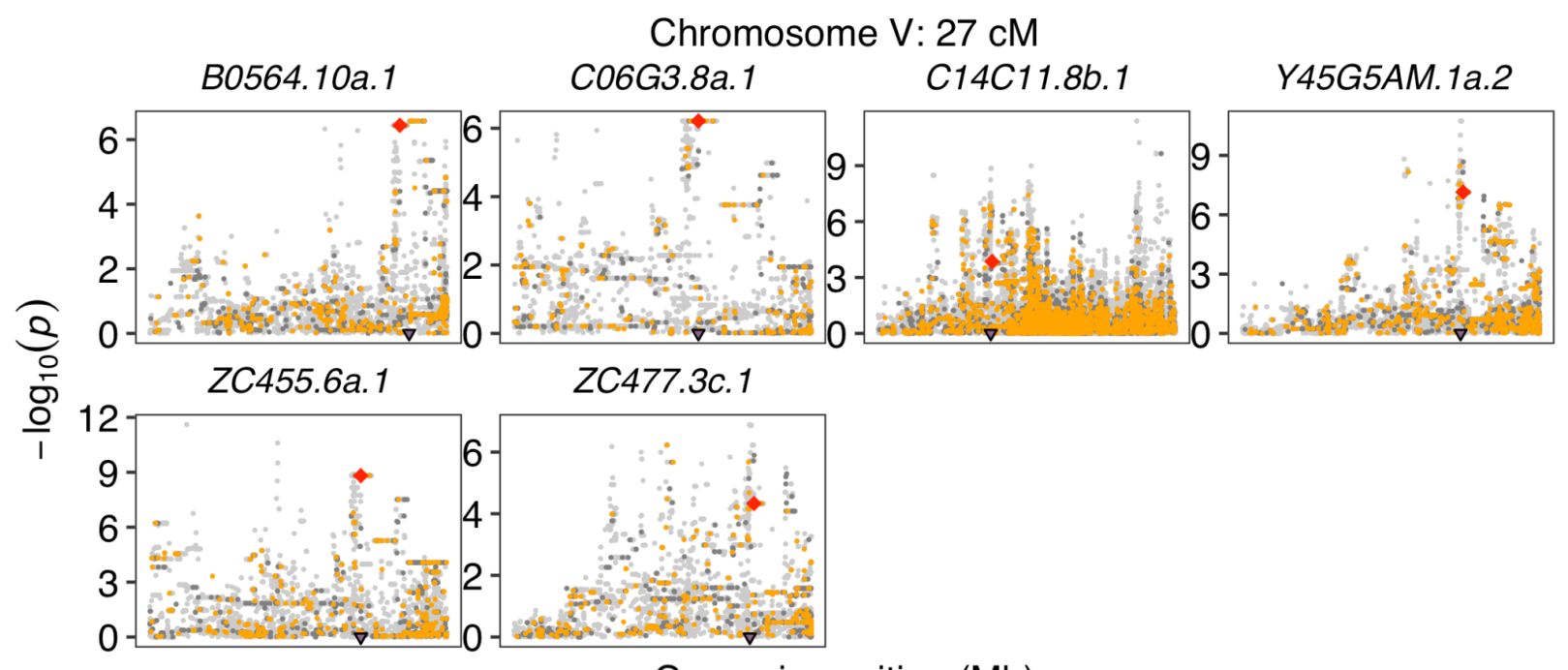

Genomic position (Mb)

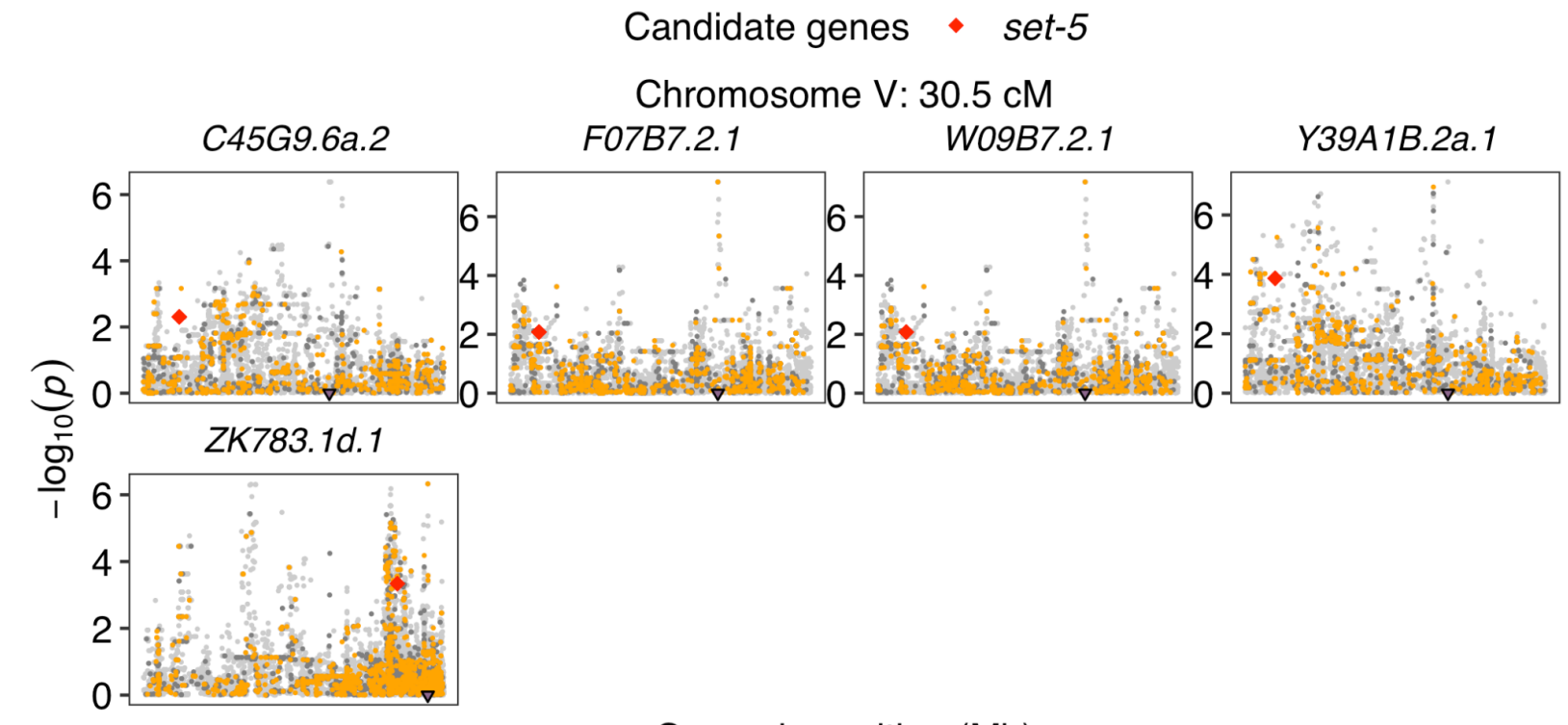

Genomic position (Mb)

120

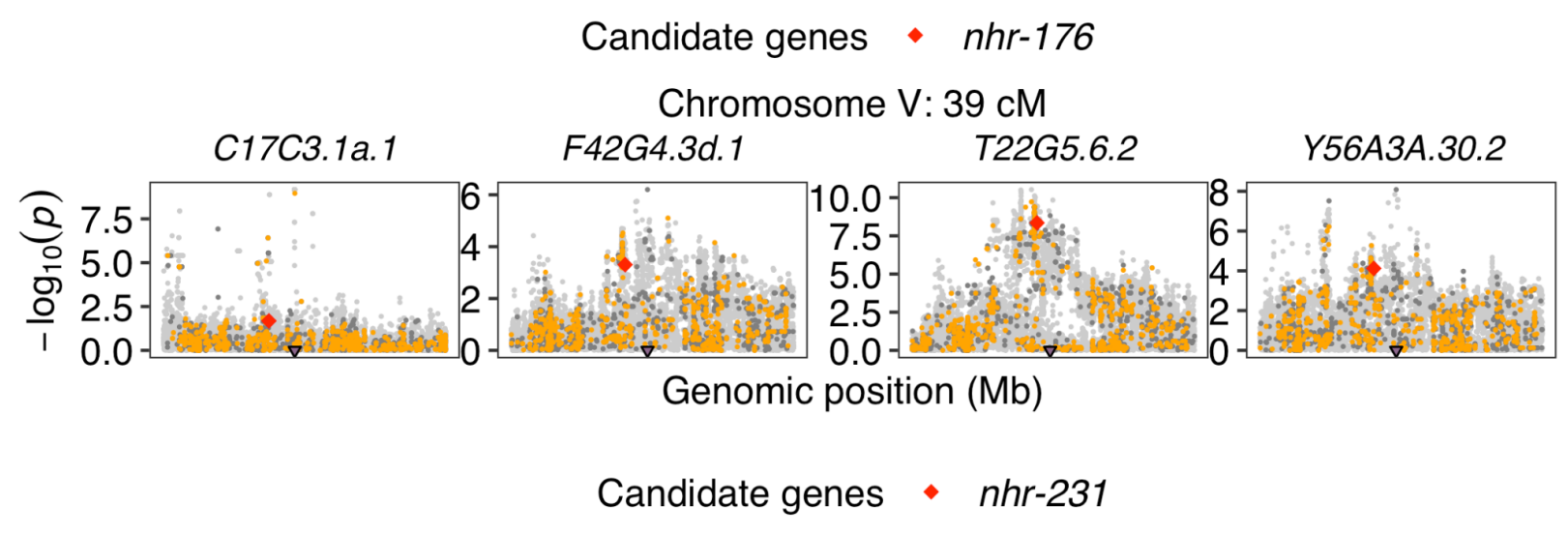




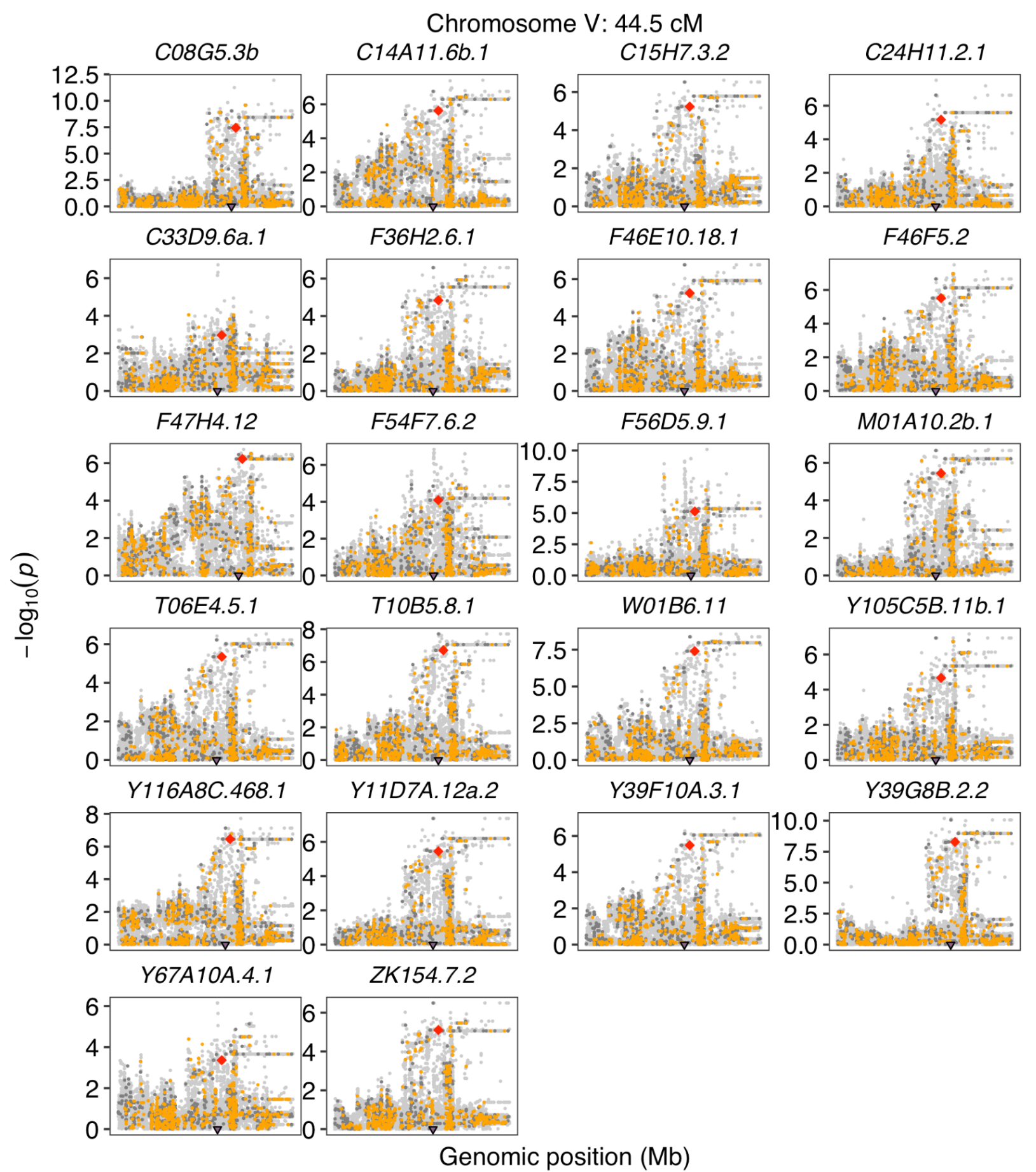

Candidate genes $t t x-1$ 


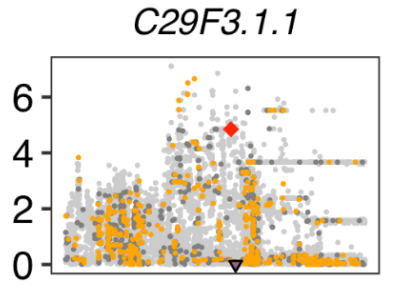

F46F5.16.1

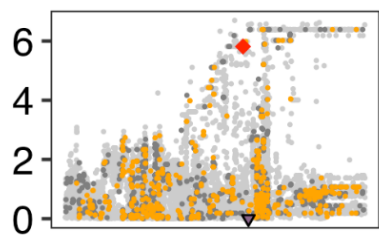

K05G3.3.1

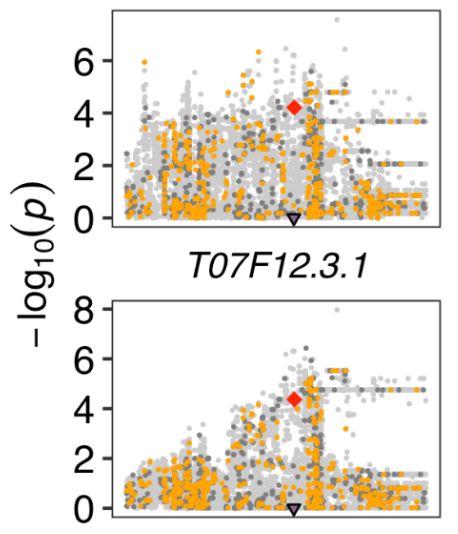

W04H10.3a.2

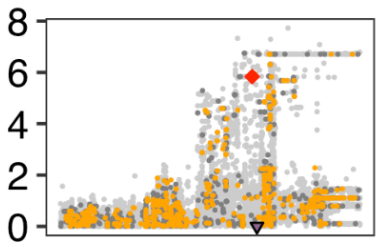

ZK262.19.1

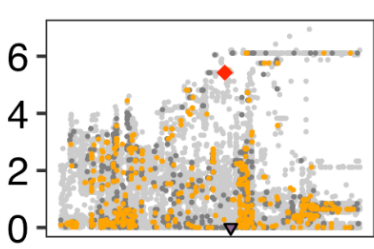

Chromosome V: $45 \mathrm{cM}$

C54G6.8.1

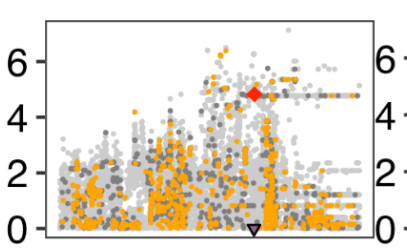

F46G10.4.1

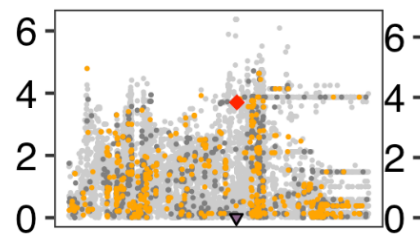

K07E3.8.2

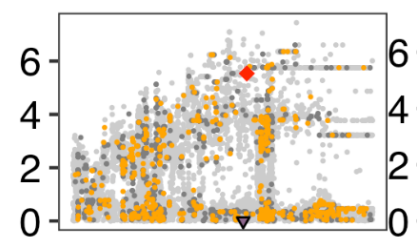

T09B4.6.1

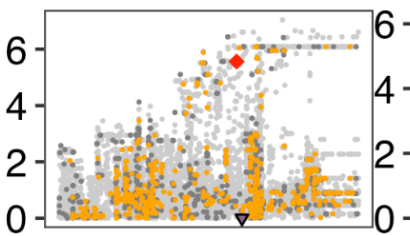

F31F4.7.1

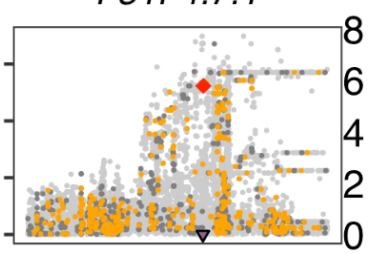

F54D7.2.2

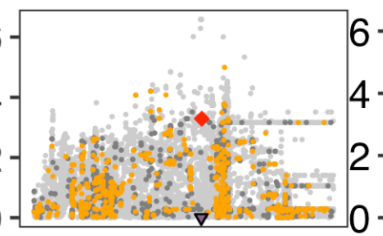

R05A10.2.1

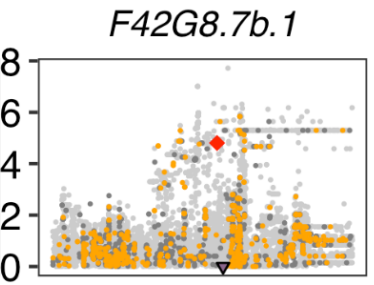

F54F7.5.2

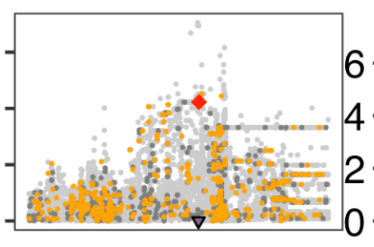

T19D7.5.1

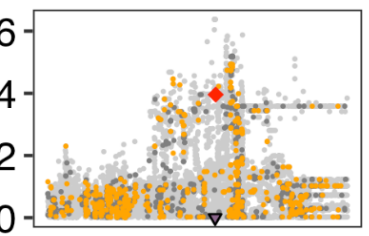

T04A6.3b. 1

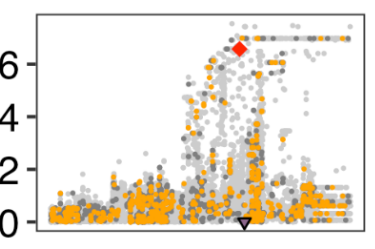

T22D1.12b.1

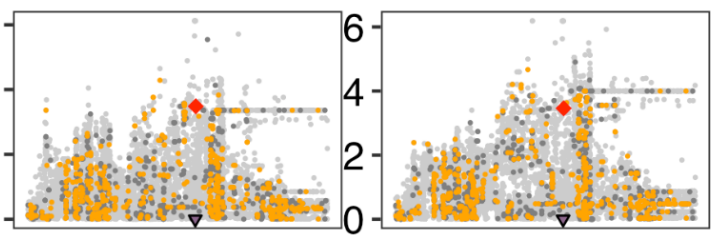

Y73B6BL.31b.1

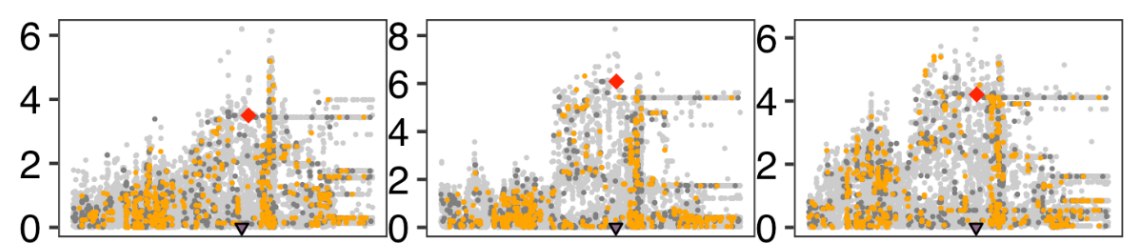

ZK666.6.1

ZK669.4.2

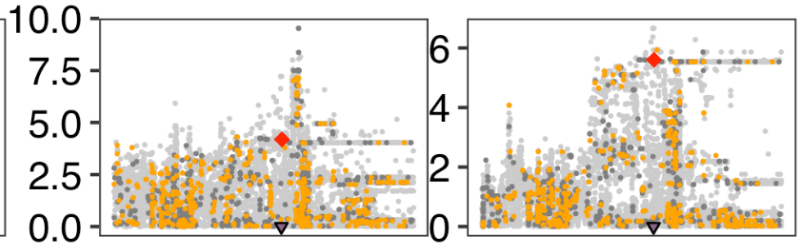

Genomic position (Mb)

Candidate genes $\quad t t x-1$ 

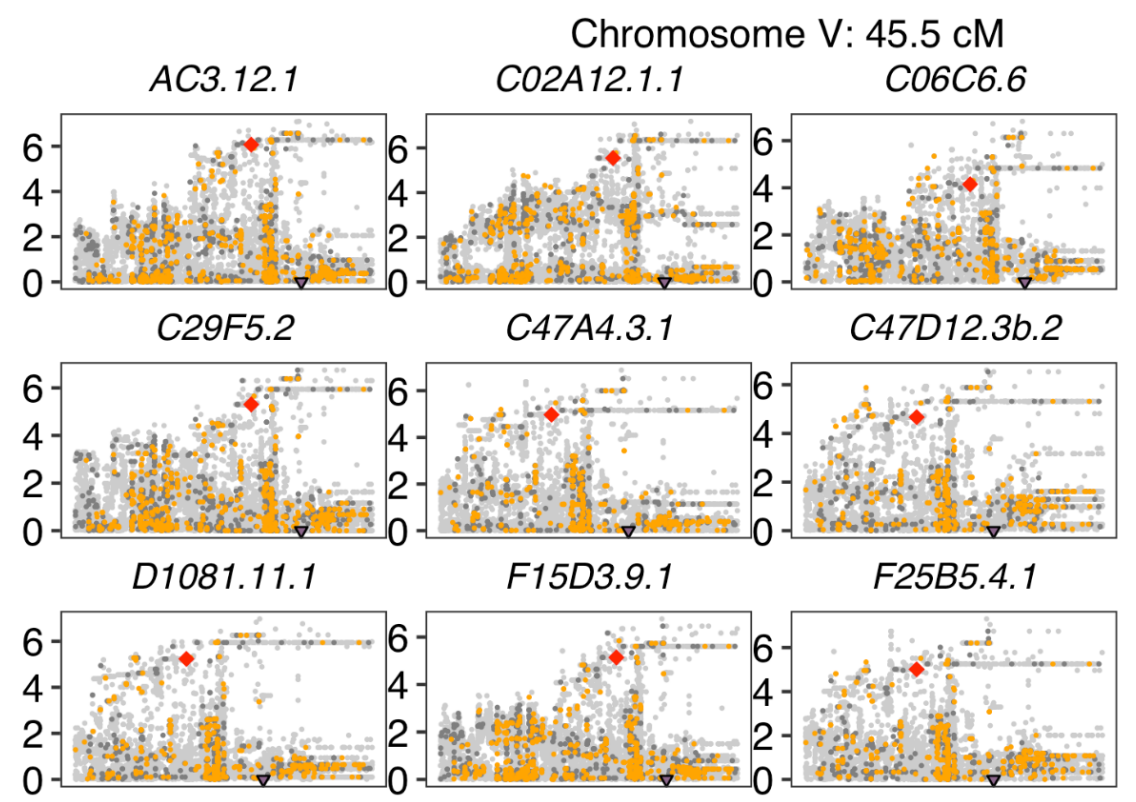

F15D3.9.1

F25B5.4.1
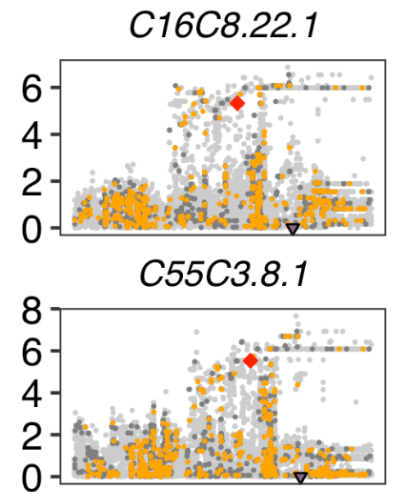

\section{F54F12.1.1}
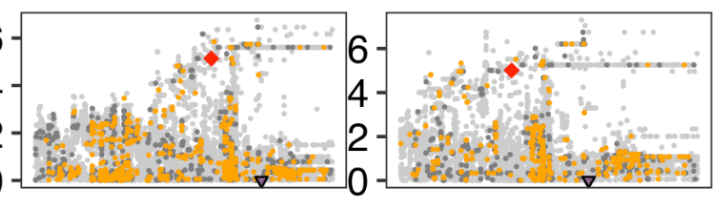

M110.4a.1

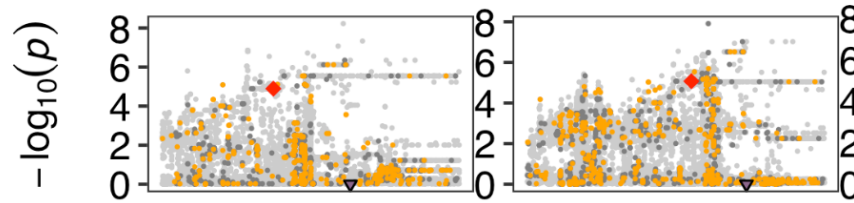

R03D7.5a. 1

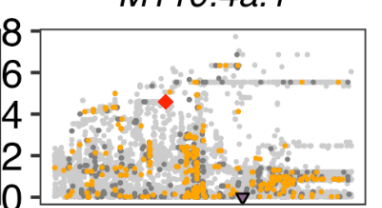

R09G11.1.1

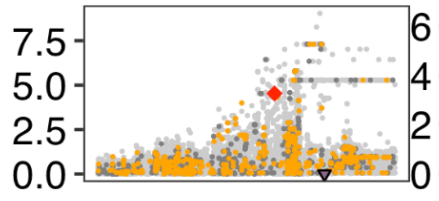

T21C9.13.1
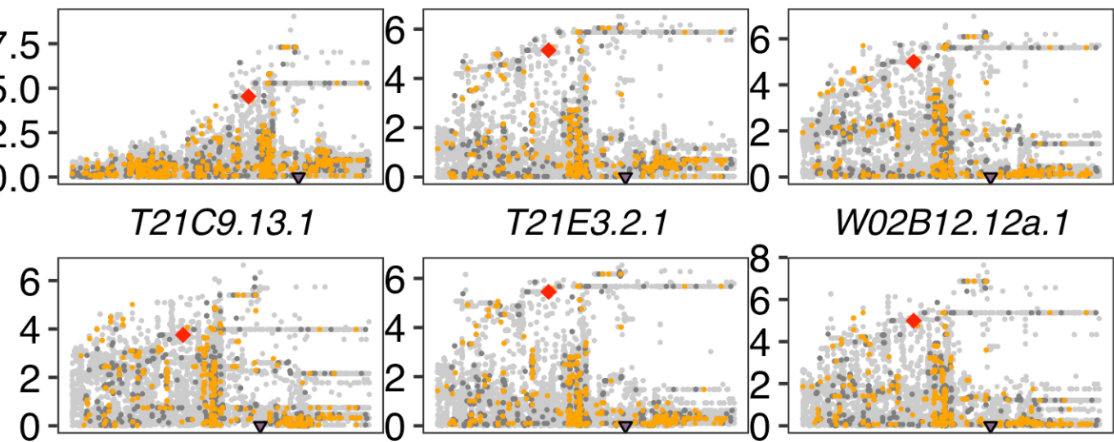

W02B12.12a.1

F26G1.8

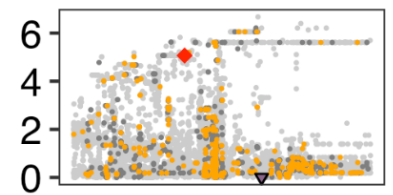

M28.4.1

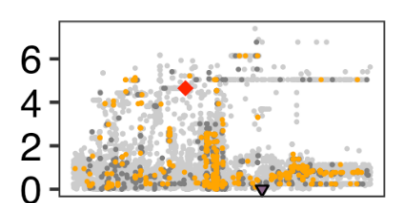

T14G10.8a.2

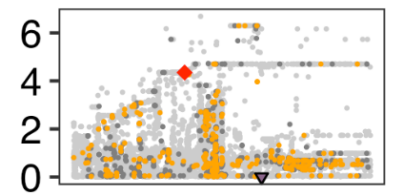

W05H5.3.1
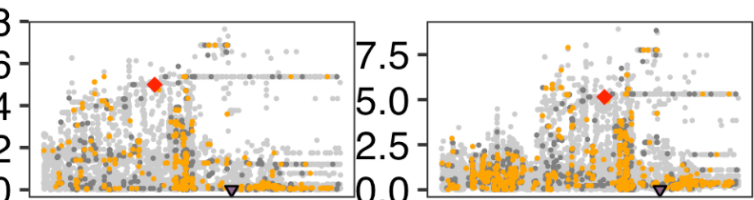

Y34B4A.3.2

Y4C6A. 1
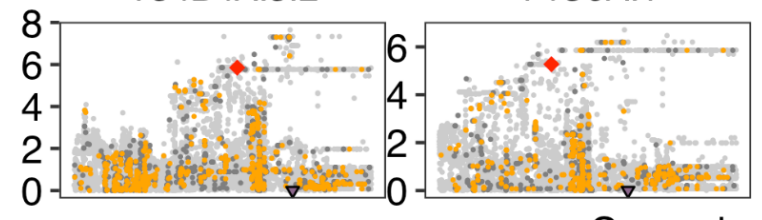

Genomic position (Mb) 


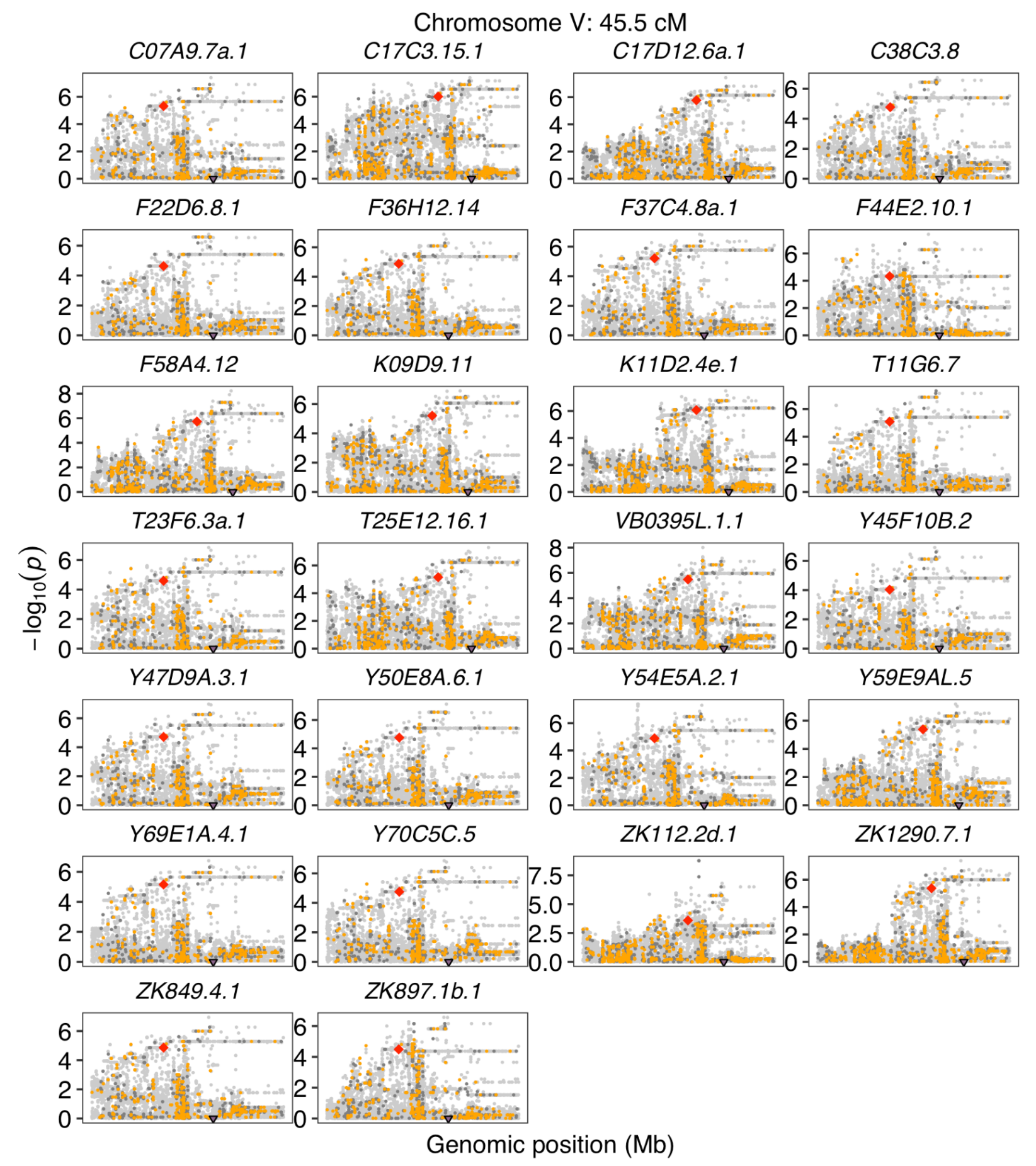


Chromosome X: $14 \mathrm{cM}$
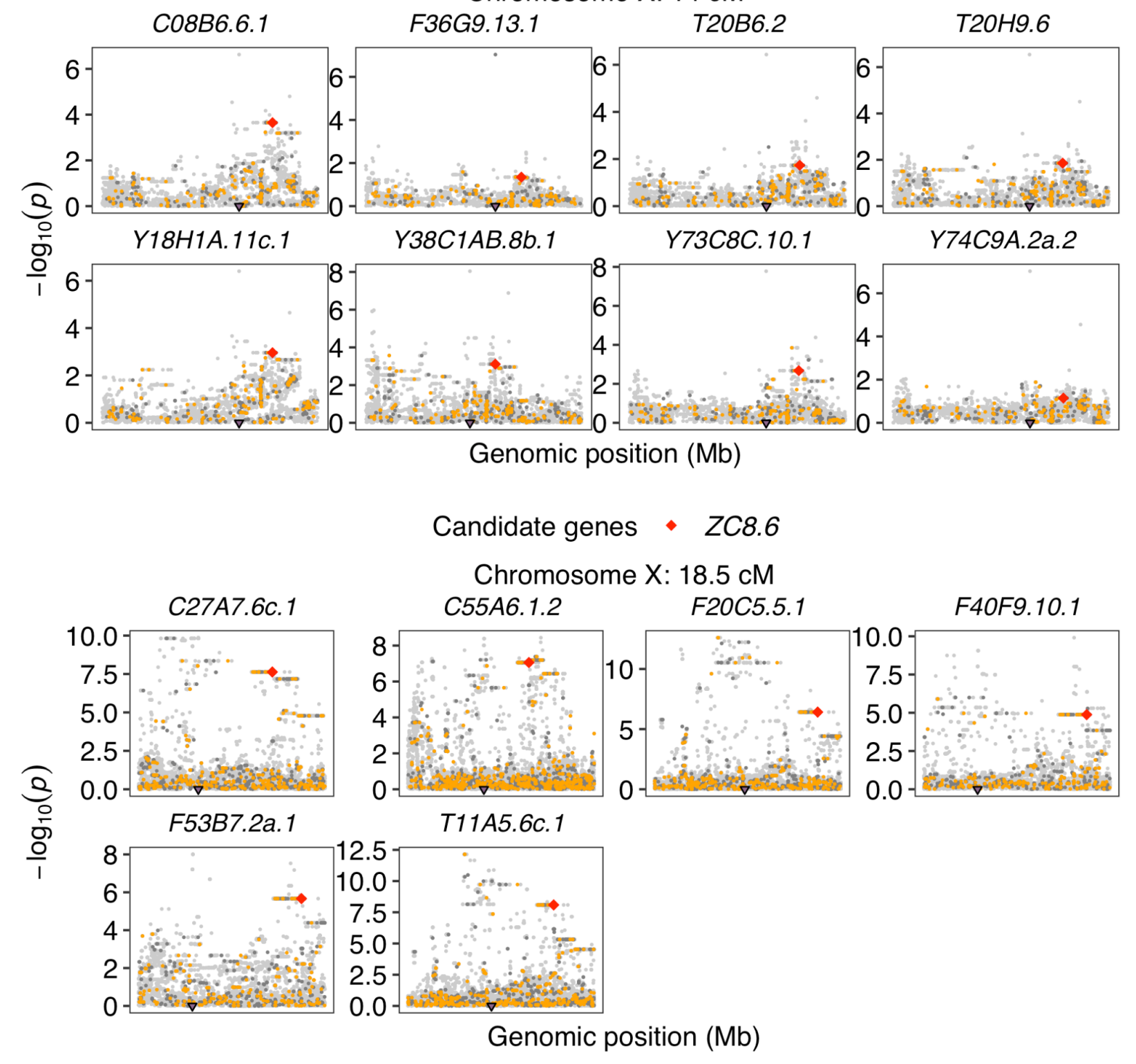

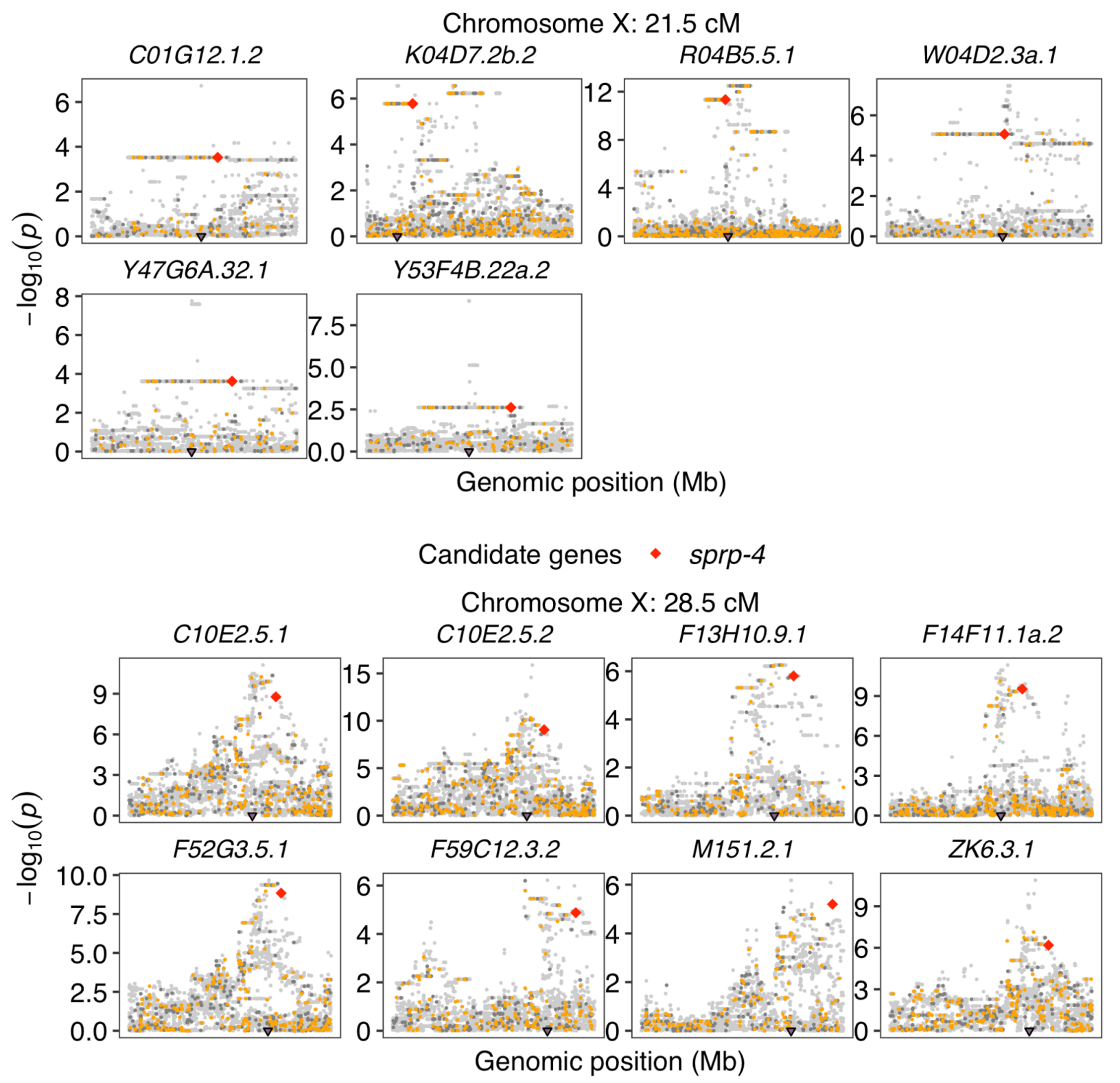


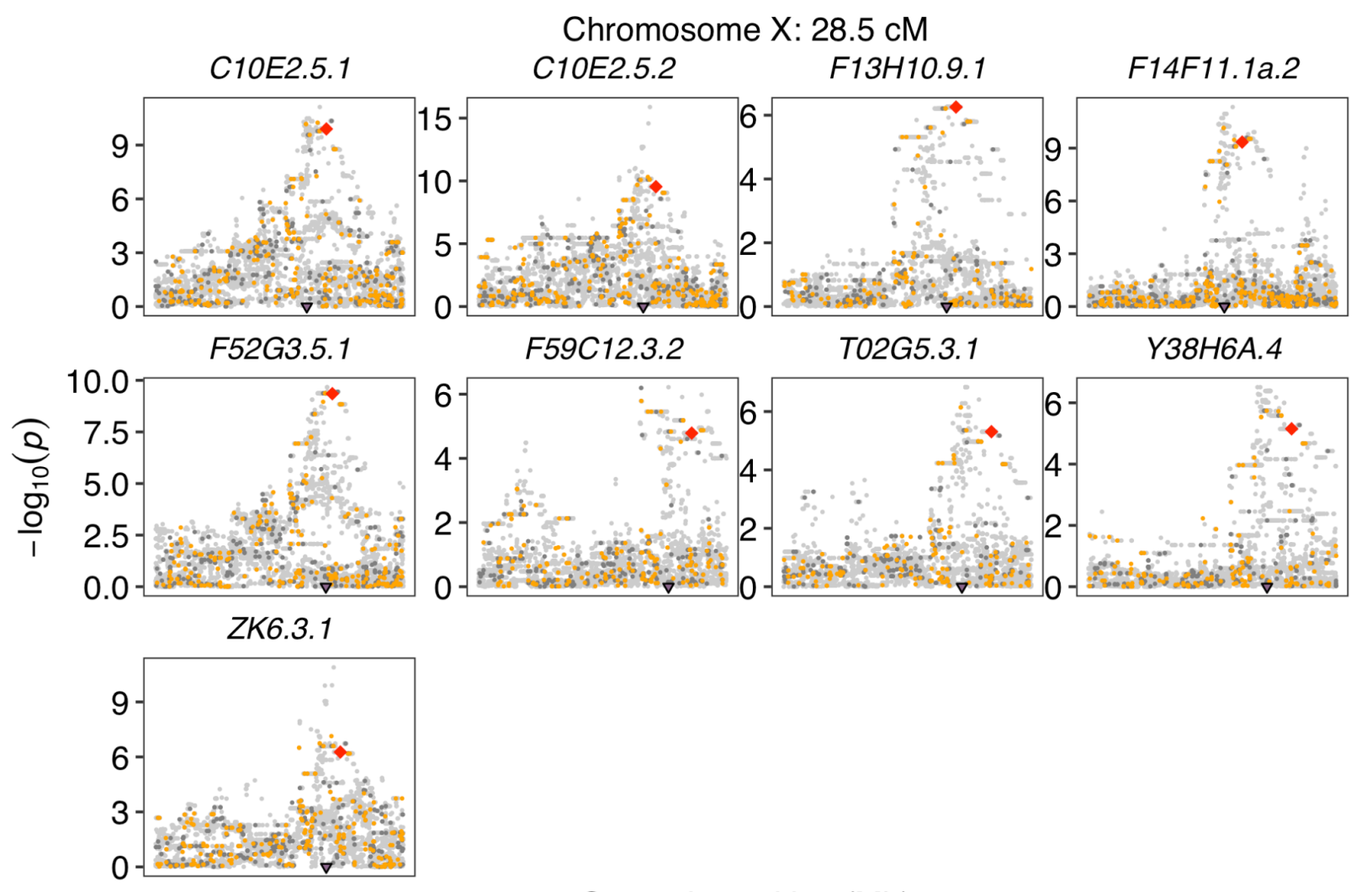

Genomic position (Mb)

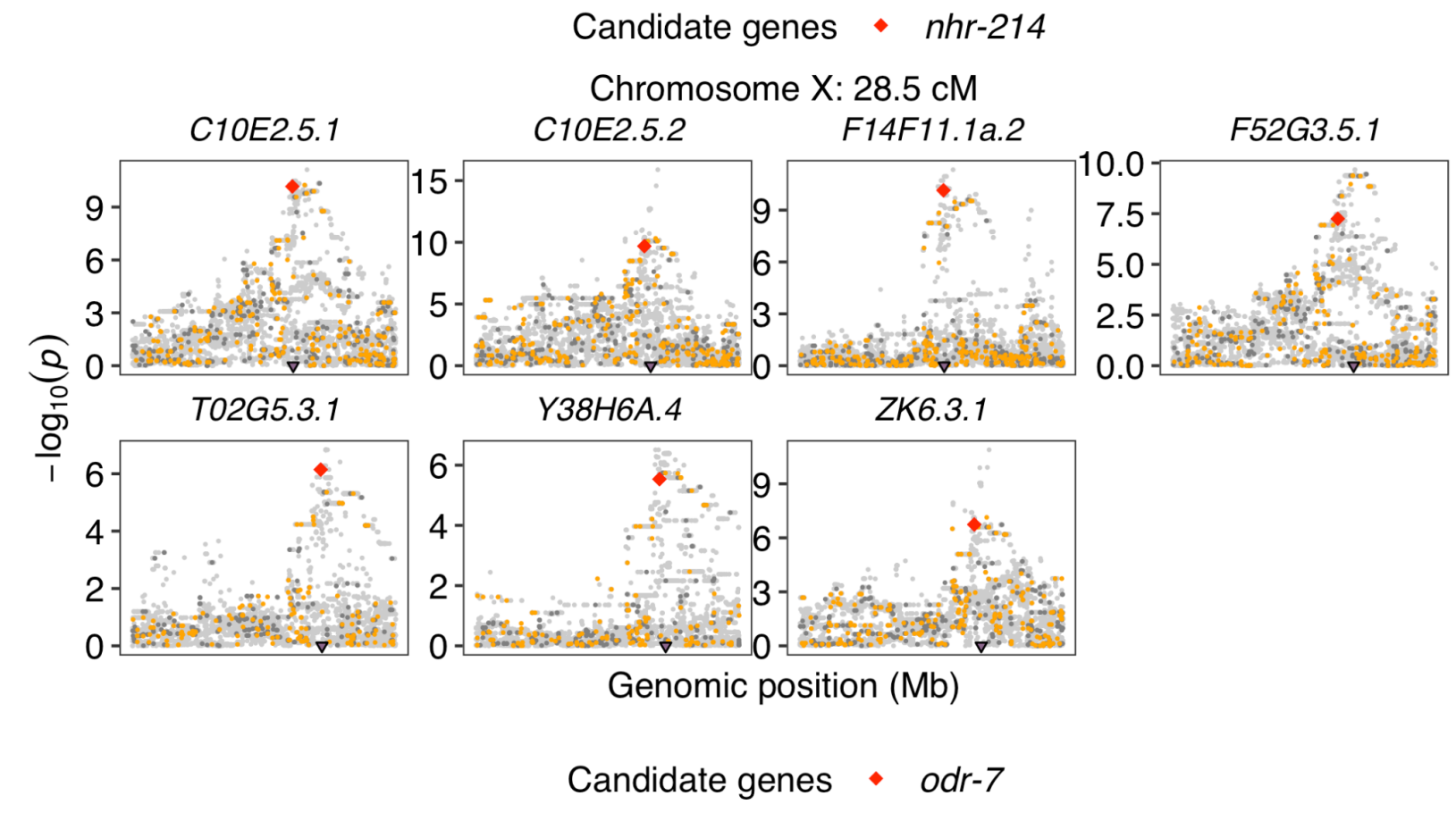




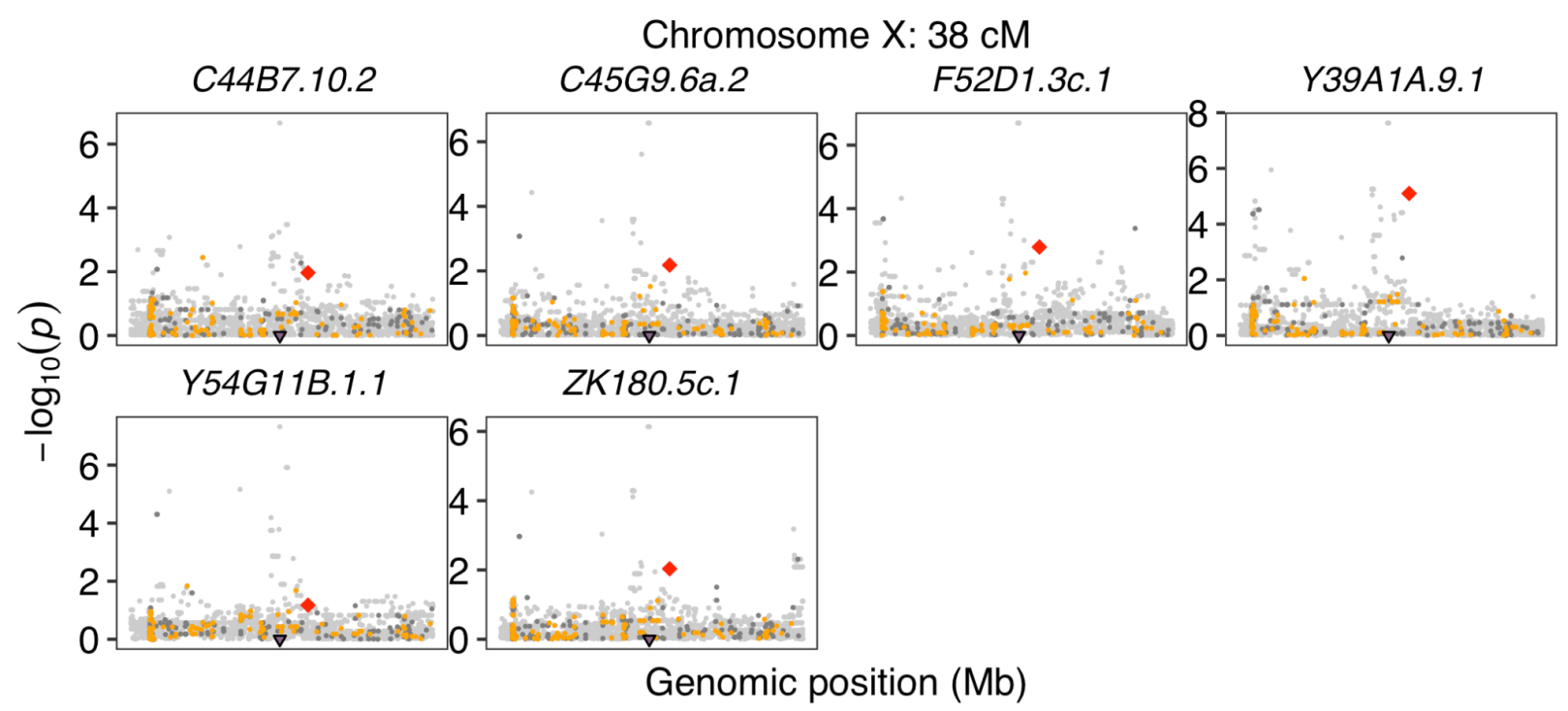

\section{Supplementary Fig. 7}

134 Fine mapping of transcript expression traits with distant eQTL in different hotspots is 135 shown. Genomic position (x-axis) is plotted against the -log10(p) values (y-axis) for each variant. Purple triangles on the $x$-axis represent eQTL positions. Candidate variants with negative BLOSUM scores in genes encoding transcription factors or chromatin cofactors are indicated as red diamonds. Other variants that are with negative BLOSUM scores, with non-negative BLOSUM scores or intergenic are colored orange, dark gray, and light gray, respectively. Transcript names of each trait are indicated above each panel. 


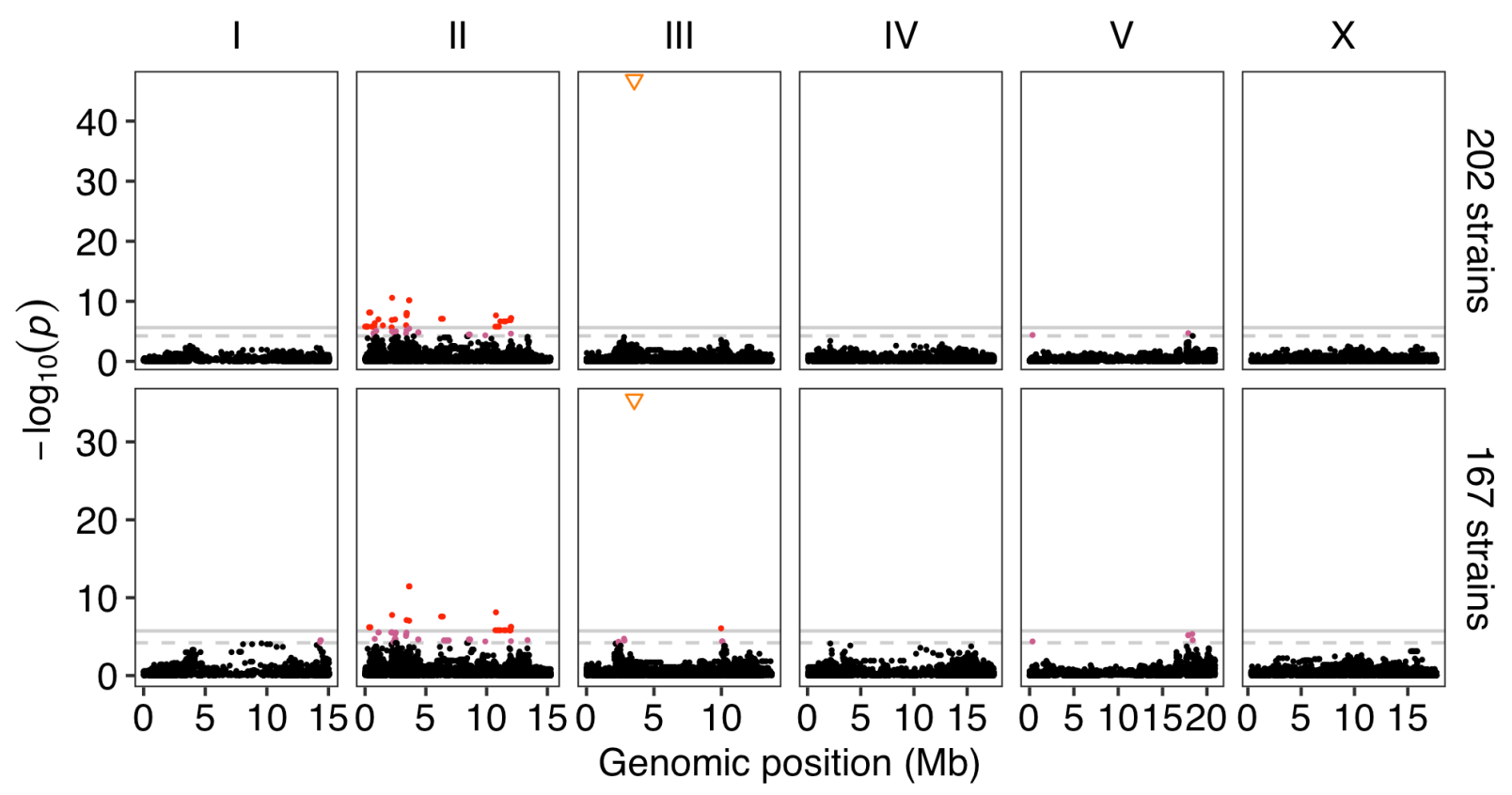

\section{Supplementary Fig. 8}

144 Manhattan plots indicating the GWA mapping result for animal length (q90.TOF) of 202 145 (top panel) and 167 (bottom panel) C. elegans wild strains in response to $\mathrm{ABZ}^{44}$ are 146 shown. Each point represents an SNV that is plotted with its genomic position ( $\mathrm{x}$-axis) 147 against its $-\log _{10}(p)$ value (y-axis) from the GWA mapping. Real SNVs that pass the 148 genome-wide EIGEN threshold (the dotted gray horizontal line) and the genome-wide 149 Bonferroni threshold (the solid gray horizontal line) are colored pink and red, respectively. 150 The pseudo SNV marker representing high allelic heterogeneity in the gene ben-1 at 151 position $3,539,640$ on chromosome III is indicated as an orange inverted triangle. 
a
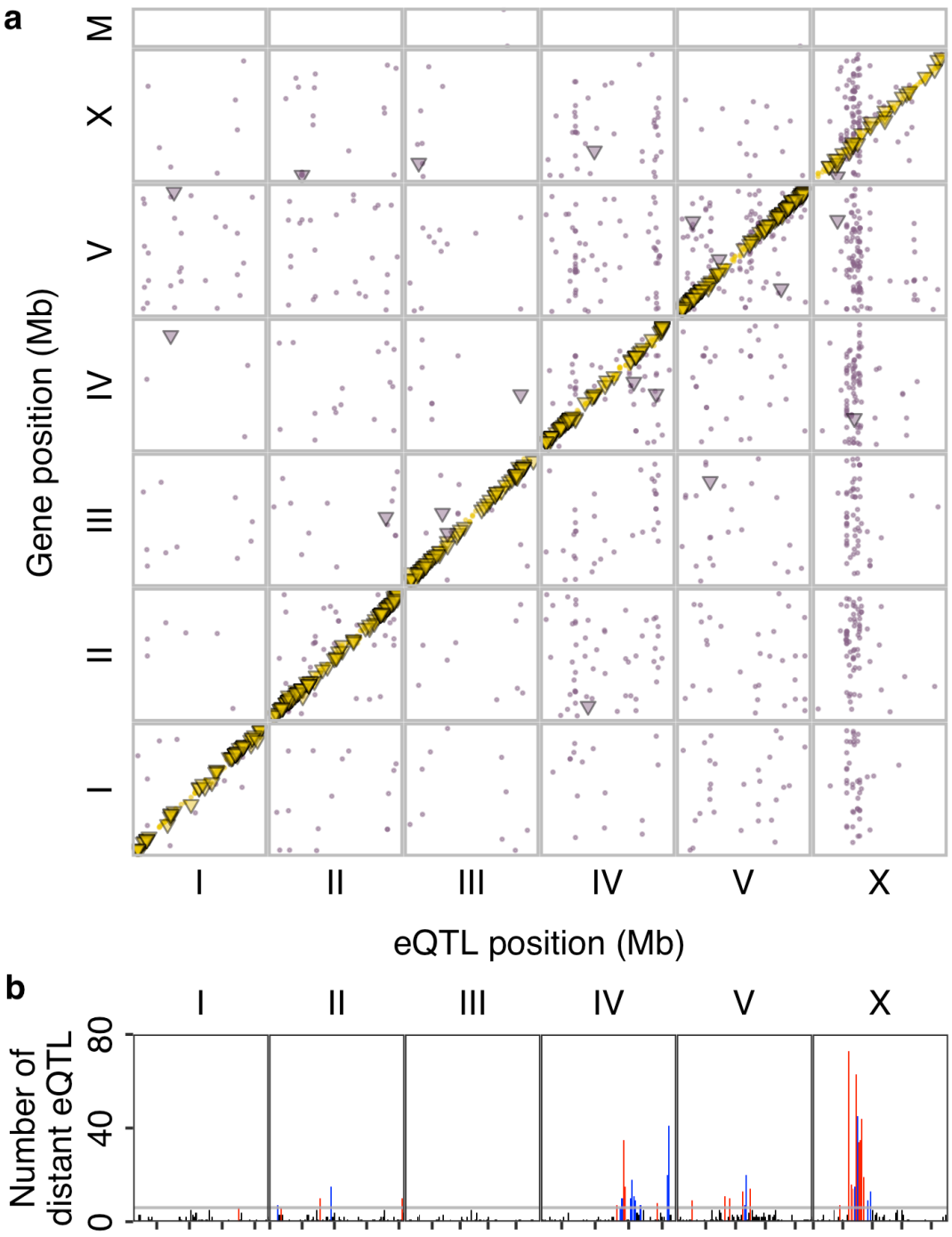

Hotspot position (cM)

Supplementary Fig. 9

\section{RIAILs eQTL.}

155 a, The genomic locations of 2,387 eQTL peaks ( $\mathrm{x}$-axis) in the RIAILs eQTL studies ${ }^{3,9}$ are plotted against the genomic locations of the 2,003 genes with expression differences $(y-$ axis). Golden points or triangles on the diagonal of the map represent local eQTL. Purple points or triangles correspond to distant eQTL. Triangles represent eQTL that were also found in our study. b. The number of distant eQTL (y-axis) in each $0.5 \mathrm{cM}$ bin across the genome (x-axis) is shown. Tick marks on the $\mathrm{x}$-axis denote every $10 \mathrm{cM}$. The horizontal gray line indicates the threshold of 6 eQTL. Bins with 6 or more eQTL were identified as hotspots and are colored red or blue. Bins with fewer than 6 eQTL are colored black. Blue bins represent hotspots that were also found in our study. 